ReV. MAt. Iberoamericana 21 (2005), no. 3, 929-996

\title{
High order regularity for subelliptic operators on Lie groups of polynomial growth
}

Nick Dungey

\begin{abstract}
Let $G$ be a Lie group of polynomial volume growth, with Lie algebra $\mathfrak{g}$. Consider a second-order, right-invariant, subelliptic differential operator $H$ on $G$, and the associated semigroup $S_{t}=e^{-t H}$. We identify an ideal $\mathfrak{n}^{\prime}$ of $\mathfrak{g}$ such that $H$ satisfies global regularity estimates for spatial derivatives of all orders, when the derivatives are taken in the direction of $\mathfrak{n}^{\prime}$. The regularity is expressed as $L_{2}$ estimates for derivatives of the semigroup, and as Gaussian bounds for derivatives of the heat kernel. We obtain the boundedness in $L_{p}, 1<p<\infty$, of some associated Riesz transform operators. Finally, we show that $\mathfrak{n}^{\prime}$ is the largest ideal of $\mathfrak{g}$ for which the regularity results hold.

Various algebraic characterizations of $\mathfrak{n}^{\prime}$ are given. In particular, $\mathfrak{n}^{\prime}=\mathfrak{s} \oplus \mathfrak{n}$ where $\mathfrak{n}$ is the nilradical of $\mathfrak{g}$ and $\mathfrak{s}$ is the largest semisimple ideal of $\mathfrak{g}$.

Additional features of this article include an exposition of the structure theory for $G$ in Section 2, and a concept of twisted multiplications on Lie groups which includes semidirect products in the Appendix.
\end{abstract}

\section{Introduction}

The heat kernel and its regularity properties play an important role in harmonic analysis on a Lie group $G$, and have been intensively studied (for comprehensive introductions see $[30,35])$. Let us mention some relevant results in the case that $G$ is a Lie group of polynomial volume growth, and $H$ is a sublaplacian on $G$.

2000 Mathematics Subject Classification: primary 22E30; secondary 35B65, 58J35.

Keywords: Lie group, subelliptic operator, heat kernel, Riesz transform, regularity estimates. 
A basic result due to Varopoulos is that the heat kernel corresponding to $H$ satisfies global Gaussian estimates for all times $t>0$ (see $[34,35,30]$ ). Saloff-Coste [31] proved that first derivatives of the kernel, in the subelliptic directions, satisfy similar estimates with an additional factor of $t^{-1 / 2}$. One might expect that $m$-th order derivatives of the kernel in the subelliptic directions can be bounded with an additional factor of $t^{-m / 2}$; this is true when $G$ is nilpotent (see $[33,22]$ ), but not always, by a counterexample of [1], when $G$ is solvable. Finally, it was proved in [23] that the $m$-th order estimates for some $m \geq 2$ are all valid if and only if $G$ is a "nilcompact" Lie group, that is, $G$ equals the local direct product of a compact Lie group and a nilpotent Lie group. (We allow the compact group to be trivial, so that every nilpotent Lie group is nilcompact.) It is worth noting that the failure of the $m$-th order estimates for $m \geq 2$ can only occur for large times $t$, since one has Gaussian estimates for all $m$ when $0<t \leq 1$ (see for example [20]).

In this paper we reconsider the question of higher order regularity for the heat kernel on a Lie group of polynomial growth, and obtain positive results for higher derivatives in certain directions. Specifically, we identify a large ideal $\mathfrak{n}^{\prime}$ of the Lie algebra of $G$ such that heat kernel derivatives of any order, in the direction of $\mathfrak{n}^{\prime}$, satisfy the expected estimates for $t \geq 1$. In case $G$ is nilpotent, or more generally, nilcompact, then $\mathfrak{n}^{\prime}$ is the whole Lie algebra and our estimates essentially reduce to known results described above.

Our results provide a new connection between analysis on $G$ and the algebraic structure of $G$. In addition, they are technically useful in applications involving the behaviour of $H$ "at infinity". For example, closely related estimates (for derivatives of order at most 2) were used in the proof of Alexopoulos that the first order Riesz tranforms are bounded in $L_{p}(1<p<\infty)$ on a group of polynomial growth: see [1, Theorem 7.7]. The estimates are also an ingredient in the large-time asymptotic expansion of the heat kernel of a complex second-order operator given in [15]. In the current paper we apply the estimates to obtain the boundedness of certain Riesz transforms "at infinity" in the direction of $\mathfrak{n}^{\prime}$.

Our basic estimates (see Theorems 1.1 and 1.2 below) are semigroup bounds in $L_{2}$ and, roughly speaking, rely on two main assumptions for the operator $H$ : (i) group-invariance, and (ii) a suitable Gårding inequality in $L_{2}$. To emphasize this generality, we give results for a general class of second-order complex-coefficient operators $H$ satisfying (i),(ii) (and not just for a sublaplacian).

To state our results we fix some notation (adapted from [30, 22, 23]). Let $G$ be a connected, simply connected Lie group of polynomial growth with Lie algebra $\mathfrak{g}$. The polynomial growth assumption is equivalent to 
the statement that $\mathfrak{g}$ is type $R$, that is, the adjoint representation of $\mathfrak{g}$ has only purely imaginary eigenvalues $([26])$. The group is unimodular and we consider the spaces $L_{p}=L_{p}(G ; d g)$ of complex-valued measurable functions where $d g$ is Haar measure for $G$; the norm of a bounded operator $T: L_{p} \rightarrow L_{q}$ is denoted $\|T\|_{p \rightarrow q}$. The left regular representation $L_{G}$ acts in $L_{p}$ and we denote by $L_{p ; \infty}=L_{p ; \infty}(G) \subseteq L_{p}$ the subspace of infinitely differentiable elements with respect to this representation (see [30], Section I.1). It follows from Sobolev embedding theorems (e.g., Appendix B of [30]) that functions in $L_{p ; \infty}$ are smooth in the classical sense.

Usually we consider $p=2$ and for $x \in \mathfrak{g}$ we have the skew-adjoint operator $d L_{G}(x)$ acting in $L_{2}$. It is often convenient to regard $d L_{G}(x)$ as a right-invariant vector field, since its restriction to $L_{2 ; \infty}$ acts as such. Let $\mathcal{R}(G)$ denote the algebra of all complex, right-invariant differential operators on $G$, acting in $L_{2 ; \infty}$. Then $\mathcal{R}(G)$ is linearly spanned by the identity operator $I$ together with all products $d L_{G}\left(x_{1}\right) \cdots d L_{G}\left(x_{m}\right)$ acting in $L_{2 ; \infty}$, where $m \geq 1, x_{1}, \ldots, x_{m} \in \mathfrak{g}$. In general $c, c^{\prime}, b, b^{\prime}$, etc., denote positive constants whose value we allow to change from line to line.

Fix a list $a_{1}, \ldots, a_{d^{\prime}} \in \mathfrak{g}$ of elements which generate the Lie algebra $\mathfrak{g}$. We consider a right-invariant operator

$$
H=-\sum_{k, l=1}^{d^{\prime}} A_{k} c_{k l} A_{l}=-\sum_{k, l=1}^{d^{\prime}} c_{k l} A_{k} A_{l}
$$

where $A_{k}=d L_{G}\left(a_{k}\right)$ and $\left(c_{k l}\right)$ is a $d^{\prime} \times d^{\prime}$ matrix of constant complex numbers satisfying the ellipticity condition

$$
\operatorname{Re} \sum_{k, l=1}^{d^{\prime}} c_{k l} \xi_{k} \overline{\xi_{l}} \geq \mu_{H} \sum_{k=1}^{d^{\prime}}\left|\xi_{k}\right|^{2}
$$

for some $\mu_{H}>0$ and all $\xi \in \mathbb{C}^{d^{\prime}}$. Consider the Sobolev space

$$
L_{2 ; 1}=\left\{\varphi \in L_{2}: A_{k} \varphi \in L_{2}, k=1, \ldots, d^{\prime}\right\}
$$

of $L_{2}$ functions once-differentiable with respect to the $A_{k}$. Then $H$ is precisely defined as the maximal accretive operator in $L_{2}$ associated with the quadratic form $q_{H}$, where

$$
q_{H}(\varphi)=\sum_{k, l=1}^{d^{\prime}}\left(c_{k l} A_{l} \varphi, A_{k} \varphi\right)
$$

for $\varphi \in L_{2 ; 1}$; thus $q_{H}(\varphi)=(H \varphi, \varphi)$ for $\varphi$ in the domain of $H$. 
The ellipticity condition gives the Gårding inequality

$$
\operatorname{Re} q_{H}(\varphi) \geq \mu_{H} \sum_{k=1}^{d^{\prime}}\left\|A_{k} \varphi\right\|_{2}^{2}
$$

for $\varphi \in L_{2 ; 1}$, which ensures that the quadratic form is sectorial with vertex at the origin. By a well-known result (see [28]) $H$ generates a contractive, holomorphic semigroup $S_{z}=e^{-z H}$ in $L_{2}$, for all $z$ in a sector $\Lambda(\Omega)=\{z \in$ $\mathbb{C}-\{0\}:|\arg z|<\Omega\}$ where $\Omega \in\langle 0, \pi / 2]$.

Let $g \mapsto|g|_{a}$ denote the modulus on $G$ associated with the vector fields $A_{1}, \ldots, A_{d^{\prime}}$ (cf. $[35,30]$ ). Let $V_{a}(t)$ be the Haar measure of the ball $\{g \in$ $\left.G:|g|_{a}<t\right\}$, for $t>0$. There are integers $D_{a} \geq 1$ and $D \geq 0$ such that

$$
c^{-1} t^{D_{a}} \leq V_{a}(t) \leq c t^{D_{a}}
$$

for $0<t \leq 1$ and

$$
c^{-1} t^{D} \leq V_{a}(t) \leq c t^{D}
$$

for $t \geq 1$; while the local dimension $D_{a}$ is determined by the choice of $a_{1}, \ldots, a_{d^{\prime}}$, the dimension at infinity $D$ is an invariant of $G$.

Local regularity results for group-invariant operators satisfying a Gårding inequality akin to (1.2) are given in [20]. In particular, the semigroup acts by convolution with a smooth kernel $K_{t}, S_{t} \varphi=K_{t} * \varphi$ for $\varphi \in L_{2}$, and the following "local" Gaussian bounds are accurate for small times $t$. Given $k \in \mathbb{N}_{0}=\{0,1,2, \ldots\}$ and $i_{1}, \ldots, i_{k} \in\left\{1, \ldots, d^{\prime}\right\}$, there exist $b, c>0$ and $\omega>0$ such that

$$
\left|\left(A_{i_{1}} \ldots A_{i_{k}} K_{t}\right)(g)\right| \leq c t^{-k / 2} t^{-D_{a} / 2} e^{\omega t} e^{-b|g|_{a}^{2} / t}
$$

for all $t>0$ and $g \in G$. Therefore, in our main results we need only consider times $t \geq 1$.

We introduce various subspaces of $\mathfrak{g}$. Let $\mathfrak{q}$ denote the radical, and $\mathfrak{n} \subseteq \mathfrak{q}$ the nilradical, of $\mathfrak{g}$ : they are respectively the largest solvable and nilpotent ideals of $\mathfrak{g}$. Let us say that a type $R$ Lie algebra $\mathfrak{h}$ is nilcompact if $\mathfrak{h}=$ $\mathfrak{h}_{s} \oplus \mathfrak{h}_{n}$ with $\mathfrak{h}_{s}, \mathfrak{h}_{n}$ respectively semisimple and nilpotent ideals of $\mathfrak{h}$ (hence $\left.\left[\mathfrak{h}_{s}, \mathfrak{h}_{n}\right]=\{0\}\right)$. The nilcompact algebras are precisely the Lie algebras of the nilcompact groups. Now $\mathfrak{n}^{\prime}$ may be defined as the largest nilcompact ideal of $\mathfrak{g}$; it is also given by $\mathfrak{n}^{\prime}=\mathfrak{s} \oplus \mathfrak{n}$, where $\mathfrak{s}$ is the largest semisimple ideal of $\mathfrak{g}$ (the proof is given in the Appendix). Note that $\mathfrak{s} \cap \mathfrak{q}=\{0\}$. Finally, the nilshadow $\mathfrak{q}_{N}$ is a nilpotent Lie algebra such that $\mathfrak{q}_{N}=\mathfrak{q}$ as vector spaces (the definition is given in $[1,2]$ and in Section 2 below). Let $\mathfrak{q}_{N ; j}, j \in \mathbb{N}$, denote the lower central series of $\mathfrak{q}_{N}$, that is, $\mathfrak{q}_{N ; 1}=\mathfrak{q}_{N}$ and 
$\mathfrak{q}_{N ; j+1}=\left[\mathfrak{q}_{N}, \mathfrak{q}_{N ; j}\right]_{N}$ where $[\cdot, \cdot]_{N}$ is the bracket of $\mathfrak{q}_{N}$. The subspaces $\mathfrak{q}, \mathfrak{n}$, $\mathfrak{s}, \mathfrak{n}^{\prime}, \mathfrak{q}_{N ; j}$ are invariants of $\mathfrak{g}$, and characteristic ideals of $\mathfrak{g}$ (that is, they are fixed by any automorphism of $\mathfrak{g})$. For the $\mathfrak{q}_{N ; j}$ this remark is not obvious and is proved in the Appendix.

Our first theorem gives regularity for derivatives in the direction of $\mathfrak{n}$, plus one derivative in the subelliptic directions. The degree of regularity depends on the position in the filtration $\mathfrak{q}_{N ; 1} \supseteq \mathfrak{q}_{N ; 2} \supseteq \mathfrak{q}_{N ; 3} \supseteq \ldots$ of $\mathfrak{q}_{N}$.

Theorem 1.1 Let $m \in \mathbb{N}_{0}$ and $y_{1}, \ldots, y_{m} \in \mathfrak{n}$ and set $Y_{i}=d L_{G}\left(y_{i}\right)$. Choose $j_{1}, \ldots, j_{m} \in \mathbb{N}=\{1,2,3, \ldots\}$ such that $y_{i} \in \mathfrak{q}_{N ; j_{i}}$ for all $i \in\{1, \ldots, m\}$, and put $w=j_{1}+\cdots+j_{m}$. Then there exists $c>0$ such that

$$
\left\|Y_{1} \cdots Y_{m} S_{t}\right\|_{2 \rightarrow 2}+t^{1 / 2}\left\|Y_{1} \cdots Y_{m} A_{k} S_{t}\right\|_{2 \rightarrow 2} \leq c t^{-w / 2} \leq c t^{-m / 2}
$$

for all $t \geq 1$ and $k \in\left\{1, \ldots, d^{\prime}\right\}$.

Note that for any $y_{1}, \ldots, y_{m} \in \mathfrak{n}$ we can always choose $j_{1}=\cdots=j_{m}=1$ and $w=m$ in the above theorem, because $\mathfrak{n} \subseteq \mathfrak{q}=\mathfrak{q}_{N ; 1}$ as subspaces. When $w>m$ the theorem gives a more precise bound.

Observe that if $G$ is solvable, that is, $\mathfrak{g}=\mathfrak{q}$, then $\mathfrak{s}=\{0\}$ because $\mathfrak{s}$ is semisimple. Thus $\mathfrak{n}=\mathfrak{n}^{\prime}$ in this case. It is quite possible to have $\mathfrak{s}=\{0\}$ even when $G$ is not solvable. Nevertheless, the following estimate giving an exponential decrease for $\mathfrak{s}$-derivatives is of interest.

Theorem 1.2 Let $y \in \mathfrak{s}$, set $Y=d L_{G}(y)$, and let $P, Q \in \mathcal{R}(G)$. Then there exist positive constants $c, \sigma, b$ such that

$$
\left\|P Y Q S_{t}\right\|_{2 \rightarrow 2} \leq c e^{-\sigma t}
$$

and

$$
\left|\left(P Y Q K_{t}\right)(g)\right| \leq c e^{-\sigma t} e^{-b|g|_{a}^{2} / t}
$$

for all $t \geq 1$ and $g \in G$.

We remark that the crucial feature of $\mathfrak{s}$, used to prove Theorem 1.2 , is that the corresponding Lie subgroup $G_{\mathfrak{s}}$ is compact and normal in $G$.

Combining the above theorems we obtain

Corollary 1.3 Let $m \in \mathbb{N}_{0}, y_{1}, \ldots, y_{m} \in \mathfrak{n}^{\prime}$ and set $Y_{i}=d L_{G}\left(y_{i}\right)$. Choose $j_{1}, \ldots, j_{m} \in \mathbb{N}$ such that $y_{i} \in \mathfrak{s} \oplus \mathfrak{q}_{N ; j_{i}}$ for all $i \in\{1, \ldots, m\}$, and put $w=j_{1}+\cdots+j_{m}$. Then there is $c>0$ such that

$$
\left\|Y_{1} \cdots Y_{m} S_{t}\right\|_{2 \rightarrow 2}+t^{1 / 2}\left\|Y_{1} \cdots Y_{m} A_{k} S_{t}\right\|_{2 \rightarrow 2} \leq c t^{-w / 2} \leq c t^{-m / 2}
$$

for all $t \geq 1$ and $k \in\left\{1, \ldots, d^{\prime}\right\}$. 
Indeed, if $y_{1}, \ldots, y_{m}$ are as in the statement of the corollary then we can write $y_{i}=w_{i}+z_{i}$ where $w_{i} \in \mathfrak{n} \cap \mathfrak{q}_{N ; j_{i}}$ and $z_{i} \in \mathfrak{s}$. Then $Y_{1} \cdots Y_{m}$ equals $W_{1} \cdots W_{m}$ modulo terms of the form $P Z_{i} Q$ where $W_{i}=d L_{G}\left(w_{i}\right)$, $Z_{i}=d L_{G}\left(z_{i}\right)$ and $P, Q \in \mathcal{R}(G)$. Thus Corollary 1.3 follows by adding the estimates of Theorems 1.1 and 1.2.

Next we consider kernel bounds. Let us say that $K_{t}$ satisfies global Gaussian bounds if for some $b, c>0$ one has

$$
\left|K_{t}(g)\right| \leq c V_{a}(t)^{-1 / 2} e^{-b|g|_{a}^{2} / t}
$$

for all $t>0$ and $g \in G$.

Theorem 1.4 Suppose that the kernel $K_{t}$ of $S_{t}$ satisfies global Gaussian bounds (1.4), and let $m, y_{1}, \ldots, y_{m} \in \mathfrak{n}^{\prime}, w$ be as in the statement of Corollary 1.3. Then there exist $c, b>0$ such that

$$
\left|\left(Y_{1} \cdots Y_{m} K_{t}\right)(g)\right|+t^{1 / 2}\left|\left(Y_{1} \cdots Y_{m} A_{k} K_{t}\right)(g)\right| \leq c t^{-w / 2} t^{-D / 2} e^{-b|g|_{a}^{2} / t}
$$

for all $t \geq 1, k \in\left\{1, \ldots, d^{\prime}\right\}$ and $g \in G$.

As an interesting application of the above results, we consider certain Riesz transforms "at infinity." These operators possess kernels which are locally integrable (and even bounded) but are not in general integrable at infinity. Our definition follows Alexopoulos (see [1], Section 8, and [2], Section 1). If $n \in \mathbb{N}$ we note the formal identity $H^{-n / 2}=\Gamma(n / 2)^{-1} \int_{0}^{\infty} d t t^{(n / 2)-1} S_{t}$, and define an operator

$$
H^{-n / 2, \infty}=\frac{1}{\Gamma(n / 2)} \int_{1}^{\infty} d t t^{(n / 2)-1} S_{t}
$$

Theorem 1.5 Assume the hypotheses and notation of Theorem 1.4, with $m \geq 1$. Then the transforms $Y_{1} \cdots Y_{m} H^{-w / 2, \infty}$ and $H^{-w / 2, \infty} Y_{1} \cdots Y_{m}$ are bounded in $L_{p}$ for $1<p<\infty$, and from $L_{1}$ to weak- $L_{1}$.

In case $G$ is nilpotent, Theorem 1.5 can be derived from the fact that the Riesz transforms $A_{i_{1}} \cdots A_{i_{k}} H^{-k / 2}$ and $H^{-k / 2} A_{i_{1}} \cdots A_{i_{k}}$ are bounded for all $k \in \mathbb{N}$ (for the latter fact see [29, 22]). On a general group of polynomial growth, these transforms are bounded when $k=1$ but not necessarily for $k \geq 2$ (for precise results see $[1,23]$ ).

Our final result shows that the estimates of Corollary 1.3 and Theorem 1.4 fail outside $\mathfrak{n}^{\prime}$. For $x \in \mathfrak{g}$, let $\mathfrak{g}_{x}$ denote the smallest ideal of $\mathfrak{g}$ which contains $x$. We denote the difference of two sets $A-B=\{x \in A: x \notin B\}$. 
Theorem 1.6 Suppose that the kernel $K_{t}$ of $S_{t}$ satisfies Gaussian bounds (1.4), and suppose $x \in \mathfrak{g}-\mathfrak{n}^{\prime}$. Then there exists $y \in \mathfrak{g}_{x} \cap \mathfrak{n}$ with the following property. For each $j \in \mathbb{N}$ there is $c(j)>0$ such that

$$
\left\|X^{j} Y S_{t}\right\|_{2 \rightarrow 2} \geq c(j) t^{-1 / 2}
$$

for all $t \geq 1$, where $X=d L_{G}(x), Y=d L_{G}(y)$.

Theorem 1.6 implies the following maximality result for $\mathfrak{n}^{\prime}$. If $\mathfrak{h}$ is an ideal of $\mathfrak{g}$ such that for all $y_{1}, y_{2} \in \mathfrak{h}$ there is $c=c\left(y_{1}, y_{2}\right)>0$ such that

$$
\left\|Y_{1} Y_{2} S_{t}\right\|_{2 \rightarrow 2} \leq c t^{-1}
$$

for all $t \geq 1$ (where $Y_{i}=d L_{G}\left(y_{i}\right)$ ), then $\mathfrak{h} \subseteq \mathfrak{n}^{\prime}$. In particular, one obtains a variation of a result of [23]: if (1.5) holds for all $y_{1}, y_{2} \in \mathfrak{g}$, then $\mathfrak{g}=\mathfrak{n}^{\prime}$, in other words, the group $G$ is nilcompact. These remarks are also true if one replaces (1.5) by the weaker condition

$$
\liminf _{t \rightarrow \infty} t^{1 / 2}\left\|Y_{1} Y_{2} S_{t}\right\|_{2 \rightarrow 2}=0
$$

Let us make some further remarks about Theorems 1.1 - 1.6.

(a) The bounds (1.4) hold in the case of a sublaplacian $H=-\sum_{k=1}^{d^{\prime}} A_{k}^{2}$. They actually hold for all second-order, complex coefficient $H$ of the type considered, as proved in $[21,15]$ using techniques of homogenization theory. To demonstrate the independence of Theorems 1.1, 1.2 and Corollary 1.3 from this result, we chose to state (1.4) as a separate assumption.

Note that, unlike the papers $[1,2,15]$ dealing with analysis on Lie groups of polynomial growth, the current paper does not rely on ideas from homogenization theory. Our approach is instead based on commutator arguments and a careful exploitation of the algebraic structure.

(b) We assume that $G$ is simply connected to avoid some algebraic complications. Nevertheless, the above theorems extend to non-simply connected groups: see Section 9.

(c) Theorem 1.1 extends to a class of second-order, subelliptic systems: see Section 9.

The higher-order regularity in the directions of $\mathfrak{n}^{\prime}$ should extend to a large class of hypoelliptic, group-invariant operators over $G$, but we do not attempt such generalizations here. 
(d) The following remark will be amplified at the end of Subsection 4.1. Suppose that $G$ is a stratified nilpotent group and that $H$ is homogeneous of order 2 on $G$ (cf. [25]). Then $\mathfrak{g}=\mathfrak{n}$ and the proof of Theorem 1.1 yields $L_{2}$ regularity estimates for derivatives of arbitary order, in arbitary directions. Together with standard Sobolev embedding, scaling and perturbation techniques, we can deduce that $K_{t}$ and all its derivatives satisfy global Gaussian bounds.

While this result is not new, the above proof differs from related proofs in $[3,16,18]$ because it is independent of the results of HelfferNourrigat [27].

(e) The estimates of Theorem 1.1, Corollary 1.3 and Theorem 1.4 remain valid if the subelliptic derivative $A_{k}$ is replaced by $X=d L_{G}(x)$ for an arbitary $x \in \mathfrak{g}$. In the solvable case $\mathfrak{g}=\mathfrak{q}$, this can be deduced as follows: since $a_{1}, \ldots, a_{d^{\prime}}$ generate $\mathfrak{g}$ we have that $\mathfrak{g}$ is linearly spanned by $a_{1}, \ldots, a_{d^{\prime}}$ together with the subspace $[\mathfrak{g}, \mathfrak{g}]$. But $[\mathfrak{g}, \mathfrak{g}] \subseteq \mathfrak{n}$ by solvability, so we can write $x=\sum_{i=1}^{d^{\prime}} c_{i} a_{i}+n$ for some $c_{i} \in \mathbb{R}$ and $n \in \mathfrak{n}$. The results follow.

In the general case, a more sophisticated argument is required: see [15], or for alternative arguments in the case $m=0$ and $H$ is a sublaplacian, the references $[1,2]$. Actually, it is shown more generally in [15] that the estimates remain valid with $A_{k}$ replaced by any monomial $X_{1} \cdots X_{s}$, where $s \in \mathbb{N}, X_{i}=d L_{G}\left(x_{i}\right), x_{i} \in \mathfrak{g}$.

The paper is organized as follows. In Section 1.1 we outline the proof of Theorem 1.1. Section 2 is an exposition of the algebraic structure of $G$, including the construction of the nilshadow and the "shadow". We also introduce the concept of groups with stratified nilshadow (to be used in the proof of Theorem 1.1). In Section 3 we obtain preliminary $L_{2}$-estimates for rescaled versions $H_{\varepsilon}$ of $H$, under the assumption that $G$ has stratified nilshadow. In Section 4, using commutator arguments and an inductive process, we complete the proof of Theorem 1.1 in the case that $G$ has stratified nilshadow. Then by transference arguments we obtain the theorem for general $G$. Sections $5,6,7$ and 8 are devoted to the respective proofs of Theorems 1.2, 1.4, 1.5 and 1.6. In Section 9 we conclude with some remarks and extensions of the results. Finally, Section 10 is an Appendix containing Lie-algebraic results: invariance properties of $\mathfrak{q}_{N ; j}$, the ideals $\mathfrak{s}$ and $\mathfrak{n}^{\prime}$, the construction of twisted multiplications on Lie groups, and the construction of Lie algebras with stratified nilshadow. These results are needed in the main text and are, we hope, of some independent interest. 
An original feature of this paper is the introduction of the class of Lie groups (of polynomial growth) with a stratified nilshadow. We show (see the Appendix) that every Lie group of polynomial growth is isomorphic to a quotient of such a group. The groups with stratified nilshadow have a simpler form of scaling at infinity than a general polynomial growth Lie group, and so may be useful in future applications (indeed, they are used in [15]).

\subsection{Outline of Theorem 1.1}

Theorem 1.1 is basic for the results of this article. Since the proof of this theorem is rather long, for the convenience of the reader we outline here the main ideas.

(i) By use of transference arguments (Section 4.2), we reduce the proof of Theorem 1.1 to the special case that $G$ has a stratified nilshadow. In this case the nilshadow $\mathfrak{q}_{N}$ is a stratified Lie algebra in the sense of [25], and the scaling on $G$ takes a simpler form. The large $t$ estimates of the theorem are equivalent to regularity estimates for rescaled versions $H_{\varepsilon}, \varepsilon \in\langle 0,1]$, of $H$.

(ii) Following Alexopoulos [1], we introduce a nilcompact group $G_{N}$, the shadow of $G$, such that $G=G_{N}$ as manifolds (essentially, $G_{N}=M \times Q_{N}$ where $M$ is a Levi subgroup of $G$ and $Q_{N}$ is the nilpotent group with Lie algebra $\mathfrak{q}_{N}$ ).

One can write $G$-invariant vector fields as linear combinations of $G_{N^{-}}$ invariant fields and conversely, where the coefficients are functions that are constant in the direction of $\mathfrak{n}$ (or $\left.\mathfrak{n}^{\prime}\right)$.

(iii) Initial estimates: in Section 3 we give initial regularity estimates for $H_{\varepsilon}$, in terms of a fractional $G_{N}$-invariant derivative.

(iv) Iteration argument: the core of the proof occurs in Section 4.1, where we iterate the initial estimates to obtain higher regularity in the direction of $\mathfrak{n}$.

This argument is no doubt related to standard elliptic regularity proofs. A crucial step is to calculate the commutator $\left[A_{k}, P\right]$ (or a rescaled version $\left.\left[A_{k, \varepsilon}, P\right]\right)$, where $P$ is a $G_{N}$-invariant derivative in the direction of $\mathfrak{n}$. Now $A_{k}$ is a combination of $G_{N}$-invariant fields with coefficients constant in the direction of $\mathfrak{n}$ (see (ii) above), so $P$ does not act on these coefficients. In this way, the calculation reduces to a calculation of commutators in the nilpotent Lie algebra $\mathfrak{q}_{N}$.

This reduction to a nilpotent algebra allows us to estimate $\left[A_{k}, P\right]$ in terms of derivatives $P^{\prime}$ of "lower order" than $P$, with respect to a certain dictionary order. This is the key to the basic regularity estimate of Proposition 4.1 for $H_{\varepsilon}$. 


\section{Algebraic Preliminaries}

This section contains results on the algebraic structure of $G$ which are needed in the sequel. While a number of the results have appeared in [1], we take a slightly different approach using elementary representation theory which avoids the use of exponential coordinates and leads to several novelties. For example, applying Lemma 10.6 of the Appendix we obtain a global formula (2.5) relating the group products of $G$ and its shadow, and simple formulae (see Lemma 2.12) relating vector fields on the two groups. The space $\mathcal{E}_{1}$ of coefficients is defined as the space of matrix elements for a certain finite-dimensional representation.

Let us fix basic notation and results. If $V$ is a real or complex vector space we denote by $\mathcal{L}(V)$ the set of linear transformations of $V$, and write $[A, B]=A B-B A$ for $A, B \in \mathcal{L}(V)$. If $\rho: \mathfrak{h} \rightarrow \mathcal{L}(V)$ is a representation of a Lie algebra $\mathfrak{h}$ in $V$, a subspace $W \subseteq V$ is said to be $\rho$-invariant if $\rho(x)(W) \subseteq$ $W$ for all $x \in \mathfrak{h}$. The representation $\rho$ is said to be completely reducible if for each $\rho$-invariant subspace $W$ of $V$ there is a $\rho$-invariant subspace $W^{\prime}$ with $V=W \oplus W^{\prime}$. One has the following criterion (Theorem 3.16.5 of [32]): $\rho$ is completely reducible if and only if $\rho(x)$ is a semisimple transformation for every $x$ in the radical $\mathfrak{q}(\mathfrak{h})$ of $\mathfrak{h}$. In particular, $\rho$ is completely reducible if $\mathfrak{h}$ is a semisimple Lie algebra.

Let $G$ be a connected, simply connected Lie group with polynomial growth. Let $(\mathfrak{g},[\cdot, \cdot])$ be the Lie algebra of $G$ and ad the adjoint representation, $(\operatorname{ad} x) y=[x, y]$ for $x, y \in \mathfrak{g}$. The polynomial growth property is equivalent to $\mathfrak{g}$ being type $R$ [26]: we will also use this equivalence for various groups associated with $G$. When convenient we use the standard identification of $\mathfrak{g}$ with the tangent space $T_{e} G$ at the identity.

For $x \in \mathfrak{g}$, let ad $x=S(x)+K(x)$ denote the Jordan decomposition of $\operatorname{ad} x \in \mathcal{L}(\mathfrak{g})$ into its semisimple part $S(x)$ and nilpotent part $K(x)$. Then $[S(x), K(x)]=0$, there exist real polynomials $s, k$ with zero constant part such that $S(x)=s(\operatorname{ad} x)$ and $K(x)=k(\operatorname{ad} x)$, and $S(x)$ and $K(x)$ are derivations of $\mathfrak{g}$. If $\mathfrak{h}$ is a subspace of $\mathfrak{g}$ with $(\operatorname{ad} x)(\mathfrak{h}) \subseteq \mathfrak{h}$, then $S(x)(\mathfrak{h}) \subseteq \mathfrak{h}$, $K(x)(\mathfrak{h}) \subseteq \mathfrak{h}$, and we denote the restrictions to $\mathfrak{h}$ by $\operatorname{ad}_{\mathfrak{h}} x, S_{\mathfrak{h}}(x), K_{\mathfrak{h}}(x)$. Note that if $\mathfrak{h}$ is an ideal of $\mathfrak{g}$ then the restrictions are defined for all $x \in \mathfrak{g}$.

The radical $\mathfrak{q}$ and nilradical $\mathfrak{n}$ of $\mathfrak{g}$ satisfy (cf. [32], Chapter 3 )

$$
\mathfrak{n} \subseteq \mathfrak{q} \quad, \quad[\mathfrak{g}, \mathfrak{q}] \subseteq \mathfrak{n}
$$

If $x \in \mathfrak{q}$ then $\operatorname{ad} x$ is nilpotent if and only if $x \in \mathfrak{n}$; thus $\mathfrak{n}=\{x \in \mathfrak{q}: \operatorname{ad} x=$ $K(x)\}=\{x \in \mathfrak{q}: S(x)=0\}$. Recall that a Levi subalgebra of $\mathfrak{g}$ is a semisimple subalgebra $\mathfrak{m}$ such that $\mathfrak{g}=\mathfrak{m} \oplus \mathfrak{q}$. 
Finally, from Lemma 10.4 of the Appendix we have the ideals $\mathfrak{s}$ and $\mathfrak{n}^{\prime}=\mathfrak{s} \oplus \mathfrak{n}$ with the properties described there.

\subsection{The nilshadow and shadow}

The construction of the nilshadow is based on the following lemma. We will apply some standard properties of Cartan subalgebras of Lie algebras (see $[5,36])$.

Lemma 2.1 There exists a subspace $\mathfrak{v}$ of $\mathfrak{q}$ with the properties

(I) $\mathfrak{q}=\mathfrak{v} \oplus \mathfrak{n}$,

(II) The subalgebra $\mathfrak{w}$ of $\mathfrak{q}$ generated by $\mathfrak{v}$ is nilpotent.

If $\mathfrak{m}$ is a Levi subalgebra of $\mathfrak{g}$ one can choose $\mathfrak{v}$ with the additional property (III) $[\mathfrak{m}, \mathfrak{v}]=\{0\}$.

Proof. Let $\mathfrak{m}$ be a Levi subalgebra and define a subalgebra $\mathfrak{q}_{0}$ of $\mathfrak{q}$ by

$$
\mathfrak{q}_{0}=\{x \in \mathfrak{q}:[\mathfrak{m}, x]=\{0\}\} .
$$

Let $\mathfrak{h}$ be a Cartan subalgebra of $\mathfrak{q}_{0}$, that is, $\mathfrak{h} \subseteq \mathfrak{q}_{0}$ is nilpotent and equals its own normalizer $N(\mathfrak{h})=\left\{x \in \mathfrak{q}_{0}:[x, \mathfrak{h}] \subseteq \mathfrak{h}\right\}$ in $\mathfrak{q}_{0}$. We claim that

$$
\mathfrak{q}=\mathfrak{h}+\mathfrak{n}
$$

where + denotes a vector space sum which is not necessarily direct. Then the lemma follows by choosing $\mathfrak{v} \subseteq \mathfrak{h}$ such that $\mathfrak{q}=\mathfrak{v} \oplus \mathfrak{n}$; note that $[\mathfrak{m}, \mathfrak{v}]=\{0\}$ because $\mathfrak{v} \subseteq \mathfrak{q}_{0}$.

It remains to justify (2.2). Since $\mathfrak{m}$ is semisimple, the representation $\rho: \mathfrak{m} \rightarrow \mathcal{L}(\mathfrak{g})$ given by $\rho(x)=\operatorname{ad} x, x \in \mathfrak{m}$, is completely reducible. Since $\mathfrak{q}$ and $\mathfrak{n}$ are $\rho$-invariant there exists a $\rho$-invariant subspace $V$ with $\mathfrak{q}=V \oplus \mathfrak{n}$. Then $\rho(V)=[\mathfrak{m}, V]=\{0\}$ because $[\mathfrak{m}, \mathfrak{q}] \subseteq \mathfrak{n}$. Thus $V \subseteq \mathfrak{q}_{0}$ so that $\mathfrak{q}=\mathfrak{q}_{0}+\mathfrak{n}$. Therefore $\pi^{\prime}\left(\mathfrak{q}_{0}\right)=\mathfrak{q} / \mathfrak{n}$, where $\pi^{\prime}: \mathfrak{q} \rightarrow \mathfrak{q} / \mathfrak{n}$ is the quotient homomorphism. It follows that $\pi^{\prime}(\mathfrak{h})$ is a Cartan subalgebra of $\mathfrak{q} / \mathfrak{n}$, since images of Cartan subalgebras under surjective homomorphisms are Cartan subalgebras. But $\mathfrak{q} / \mathfrak{n}$ is abelian because $[\mathfrak{q}, \mathfrak{q}] \subseteq \mathfrak{n}$, and hence $\pi^{\prime}(\mathfrak{h})=\mathfrak{q} / \mathfrak{n}$. This implies (2.2).

Lemma 2.2 Let $\mathfrak{v}$ be a subspace of $\mathfrak{g}$ such that the Lie subalgebra $\mathfrak{w}$ of $\mathfrak{g}$ generated by $\mathfrak{v}$ is nilpotent. Then

$$
S(a) b=0 \quad, \quad[S(a), S(b)]=0=[S(a), K(b)]
$$

for all $a, b \in \mathfrak{v}$, and the maps $\mathfrak{v} \rightarrow \mathcal{L}(\mathfrak{g})$ given by $a \mapsto S(a), a \mapsto K(a)$, $a \in \mathfrak{v}$, are linear. 
Proof. If $x \in \mathfrak{w}$ then $\operatorname{ad}_{\mathfrak{w}} x \in \mathcal{L}(\mathfrak{w})$ has Jordan decomposition

$$
\operatorname{ad}_{\mathfrak{w}} x=S_{\mathfrak{w}}(x)+K_{\mathfrak{w}}(x)
$$

Since $\mathfrak{w}$ is nilpotent, one has $\operatorname{ad}_{\mathfrak{w}} x=K_{\mathfrak{w}}(x)$ so that $S_{\mathfrak{w}}(x)=0$. In particular $S(a) b=0$ for all $a, b \in \mathfrak{v}$.

Next, consider the complexified Lie algebras $\mathfrak{w}_{\mathbb{C}}=\mathfrak{w} \otimes \mathbb{C}, \mathfrak{g}_{\mathbb{C}}=\mathfrak{g} \otimes \mathbb{C}$ with $\mathfrak{w}_{\mathbb{C}} \subseteq \mathfrak{g}_{\mathbb{C}}$, and define a representation $\rho: \mathfrak{w}_{\mathbb{C}} \rightarrow \mathcal{L}\left(\mathfrak{g}_{\mathbb{C}}\right)$ by $\rho(x) y=[x, y]$, $x \in \mathfrak{w}_{\mathbb{C}}, y \in \mathfrak{g}_{\mathbb{C}}$. For $\lambda$ in $\mathfrak{g}_{\mathbb{C}}^{*}$ define $V_{\lambda}$ to be the subspace of all $u \in \mathfrak{g}_{\mathbb{C}}$ for which there exists $m \in \mathbb{N}$ with

$$
(\rho(x)-\lambda(x))^{m} u=0
$$

for all $x \in \mathfrak{w}_{\mathbb{C}}$. Then $V_{\lambda}$ is $\rho$-invariant. Since $\mathfrak{w}_{\mathbb{C}}$ is a complex nilpotent algebra, Theorem 3.5.8 of [32] gives a direct sum decomposition

$$
\mathfrak{g}_{\mathbb{C}}=\bigoplus_{\lambda \in \Lambda} V_{\lambda}
$$

for a finite subset $\Lambda \subseteq \mathfrak{g}_{\mathbb{C}}^{*}$. But $\rho(x)$ has Jordan decomposition $\rho(x)=$ $S(x)^{\mathbb{C}}+K(x)^{\mathbb{C}}$ for $x \in \mathfrak{w}_{\mathbb{C}}$ where superscript $\mathbb{C}$ denotes complexification of a linear transformation. From the uniqueness of Jordan decomposition in $\mathcal{L}\left(V_{\lambda}\right)$ it follows that

$$
S(x)^{\mathbb{C}} u=\lambda(x) u \quad, \quad K(x)^{\mathbb{C}} u=(\rho(x)-\lambda(x)) u
$$

whenever $\lambda \in \Lambda, u \in V_{\lambda}$ and $x \in \mathfrak{w}_{\mathbb{C}}$. Therefore $S(x)^{\mathbb{C}}$ commutes with $S(y)^{\mathbb{C}}$ and with $K(y)^{\mathbb{C}}$ for all $x, y \in \mathfrak{w}_{\mathbb{C}}$, and $x \mapsto S(x)^{\mathbb{C}}, x \mapsto K(x)^{\mathbb{C}}$ are linear maps on $\mathfrak{w}_{\mathbb{C}}$. The statements of the lemma follow by restriction to $\mathfrak{v}$.

Suppose $\mathfrak{v}$ satisfies properties (I) and (II) of Lemma 2.1. Then, as in Section 2 of $[1]$, there is a Lie bracket $[\cdot, \cdot]_{N}$ on $\mathfrak{q}$ satisfying

$$
\begin{aligned}
{\left[v_{1}, n_{1}\right]_{N} } & =K\left(v_{1}\right) n_{1}=\left[v_{1}, n_{1}\right]-S\left(v_{1}\right) n_{1}, \\
{\left[v_{1}, v_{2}\right]_{N} } & =\left[v_{1}, v_{2}\right], \\
{\left[n_{1}, n_{2}\right]_{N} } & =\left[n_{1}, n_{2}\right],
\end{aligned}
$$

for all $v_{1}, v_{2} \in \mathfrak{v}$ and $n_{1}, n_{2} \in \mathfrak{n}$. (The verification of the Jacobi identity for $[\cdot, \cdot]_{N}$ is a special case of Lemma 10.5 of the Appendix, as noted there.) The Lie algebra $\mathfrak{q}_{N}=\left(\mathfrak{q},[\cdot, \cdot]_{N}\right)$ is nilpotent and is called the nilshadow of $\mathfrak{q}$; we also call it the nilshadow of $\mathfrak{g}$.

Let $\mathfrak{q}_{N ; j}, j \in \mathbb{N}$, denote the lower central series of $\mathfrak{q}_{N}$, that is, the ideals of $\mathfrak{q}_{N}$ defined by $\mathfrak{q}_{N ; 1}=\mathfrak{q}_{N}$ and $\mathfrak{q}_{N ; j+1}=\left[\mathfrak{q}, \mathfrak{q}_{N ; j}\right]_{N}$. Let $r \in \mathbb{N}$ denote the nilpotent step of $\mathfrak{q}_{N}$, so that $\mathfrak{q}_{N ; r} \neq\{0\}$ and $\mathfrak{q}_{N ; s}=\{0\}$ for all $s \geq r+1$. 
Although the bracket $[\cdot, \cdot]_{N}$ may depend on the choice of $\mathfrak{v}$, the subspaces $\mathfrak{q}_{N ; j}$ are independent of the choice of $\mathfrak{v}$ and hence are invariants of $\mathfrak{g}$. Moreover the $\mathfrak{q}_{N ; j}$ are characteristic ideals of $\mathfrak{g}$. Proofs of these facts are given in Subsection 10.1 of the Appendix. Elementary properties of $\mathfrak{q}_{N ; j}$ are given in

Proposition 2.3 Let $\mathfrak{q}_{N}$ be defined by (2.3) relative to a subspace $\mathfrak{v}$ which satisfies conditions (I), (II) of Lemma 2.1. Then

$$
\mathfrak{q} \supseteq \mathfrak{n} \supseteq[\mathfrak{q}, \mathfrak{q}] \supseteq \mathfrak{q}_{N ; 2} \supseteq \ldots \supseteq \mathfrak{q}_{N ; r} \supseteq \mathfrak{q}_{N ; r+1}=\{0\}
$$

and

$$
\left[\mathfrak{q}_{N ; j}, \mathfrak{q}_{N ; k}\right]_{N} \subseteq \mathfrak{q}_{N ; j+k}
$$

for all $j, k \in \mathbb{N}$. One has that $S_{\mathfrak{q}}(v) \in \mathcal{L}(\mathfrak{q})$ is a derivation of $\mathfrak{q}_{N}$ for all $v \in \mathfrak{v}$. If $\mathfrak{v}$ satisfies (III) of Lemma 2.1 then $\operatorname{ad}_{\mathfrak{q}}(m)$ is a derivation of $\mathfrak{q}_{N}$ for all $m \in \mathfrak{m}$.

If $U \subseteq \mathfrak{q}_{N}$ is a subspace such that $\mathfrak{q}_{N}=U+\mathfrak{q}_{N ; 2}$ then $U$ generates the Lie algebra $\mathfrak{q}_{N}$.

Proof. It follows from (2.3) that $\mathfrak{q}_{N ; 2} \subseteq[\mathfrak{q}, \mathfrak{q}]$, because $K\left(v_{1}\right)$ is a polynomial in ad $v_{1}$ with zero constant part. The remaining inclusions are from (2.1) or are obvious. The statements about derivations are straightforward to verify from Lemmas 2.1 and 2.2 .

Let $\mathfrak{a} \supseteq U$ denote the subalgebra of $\mathfrak{q}_{N}$ generated by $U$, where $U+\mathfrak{q}_{N ; 2}=\mathfrak{q}_{N}$. If $x_{1}, \ldots, x_{k} \in \mathfrak{q}_{N}$ we write $x_{i}=y_{i}+z_{i}, y_{i} \in U, z_{i} \in \mathfrak{q}_{N ; 2}$, and observe that

$$
\left[x_{1},\left[x_{2}, \cdots\left[x_{k-1}, x_{k}\right]_{N} \cdots\right]_{N}\right]_{N}-\left[y_{1},\left[y_{2}, \cdots\left[y_{k-1}, y_{k}\right]_{N} \cdots\right]_{N}\right]_{N} \in \mathfrak{q}_{N ; k+1}
$$

Since $\mathfrak{q}_{N ; r+1}=\{0\}$ it follows by backwards induction on $k$ that $\mathfrak{q}_{N ; k} \subseteq \mathfrak{a}$ for all $k \in\{1, \ldots, r\}$. In particular $\mathfrak{a}=\mathfrak{q}_{N}$ and $U$ generates $\mathfrak{q}_{N}$.

Henceforth we fix a Levi subalgebra $\mathfrak{m}$ of $\mathfrak{g}$ and fix $\mathfrak{v}$ satisfying all properties of Lemma 2.1, and consider the nilshadow $\mathfrak{q}_{N}$ defined by (2.3). We extend the bracket $[\cdot, \cdot]_{N}$ to $\mathfrak{g}$ by setting

$$
\left[x_{1}+q_{1}, x_{2}+q_{2}\right]_{N}=\left[x_{1}, x_{2}\right]+\left[q_{1}, q_{2}\right]_{N}
$$

for $x_{1}, x_{2} \in \mathfrak{m}, q_{1}, q_{2} \in \mathfrak{q}_{N}$. The Lie algebra $\mathfrak{g}_{N}=\left(\mathfrak{g},[\cdot, \cdot]_{N}\right)$ will be called the shadow of $\mathfrak{g}$. Note that $\mathfrak{g}_{N}=\mathfrak{m} \oplus \mathfrak{q}_{N}$ with $\mathfrak{m}, \mathfrak{q}_{N}$ ideals of $\mathfrak{g}_{N}$ and $\left[\mathfrak{m}, \mathfrak{q}_{N}\right]_{N}=\{0\}$. Thus the shadow is a nilcompact algebra.

Let $G_{N}$ be the connected, simply connected Lie group with Lie algebra $\mathfrak{g}_{N}$; we call $G_{N}$ the shadow of $G$. We write $*_{N}$ for the group operation of $G_{N}$ and $g^{-1_{N}}$ for the $G_{N}$-inverse of $g$. Let $Q_{N}, M$ denote the analytic 
subgroups of $G_{N}$ with Lie algebras $\mathfrak{q}_{N}, \mathfrak{m}$ respectively. They are closed, simply connected normal subgroups of $G_{N}$, with $G_{N}=M *_{N} Q_{N}$ and $M \cap$ $Q_{N}=\{e\}$. Thus $G_{N}$ is the internal direct product of $M$ and $Q_{N}$. Note that $Q_{N}$ is nilpotent and $M$ is semisimple and compact (because $\mathfrak{m}$ is a type $R$ semisimple algebra the Killing form $a \in \mathfrak{m} \rightarrow \operatorname{Tr}\left((\operatorname{ad} a)^{2}\right)$ is negative-definite, and the compactness follows by Theorem 4.11.7 of [32]).

\subsection{Comparison of Lie group and algebra structures}

In this subsection we compare the Lie structures of $G$ and $G_{N}$ and show how to identify $G=G_{N}$ as manifolds.

For $y=m+v+n \in \mathfrak{g}$ with $m \in \mathfrak{m}, v \in \mathfrak{v}, n \in \mathfrak{n}$ we define

$$
\tau_{0}(y)=\operatorname{ad}_{\mathfrak{q}}(m)+S_{\mathfrak{q}}(v) \in \mathcal{L}\left(\mathfrak{q}_{N}\right) \quad ;
$$

by Proposition 2.3 this is a derivation of $\mathfrak{q}_{N}$. From (2.1) and Lemmas 2.1 and 2.2 one verifies that $\tau_{0}: \mathfrak{g}_{N} \rightarrow \mathcal{L}\left(\mathfrak{q}_{N}\right)$ is a representation of $\mathfrak{g}_{N}$, and of $\mathfrak{g}$, in $\mathfrak{q}_{N}$. Extend $\tau_{0}(y)$ trivially to a derivation $\tau(y)$ of $\mathfrak{g}_{N}$ by setting $\tau(y)(m+q)=\tau_{0}(y) q, m \in \mathfrak{m}, q \in \mathfrak{q}$. Thus we obtain a representation

$$
\tau: \mathfrak{g}_{N} \rightarrow \mathcal{L}\left(\mathfrak{g}_{N}\right)
$$

of $\mathfrak{g}_{N}$, and of $\mathfrak{g}$, in $\mathfrak{g}_{N}$ by derivations of $\mathfrak{g}_{N}$. A straightforward calculation shows that $\tau$ relates the Lie algebras $\mathfrak{g}$ and $\mathfrak{g}_{N}$ in the sense of Lemma 10.5 of the Appendix, that is,

$$
[x, y]=[x, y]_{N}+\tau(x) y-\tau(y) x
$$

for all $x, y \in \mathfrak{g}$. Moreover $\tau$ verifies the condition $\tau(\tau(x) y)=0$ of that lemma, since $\tau(x) y \in \mathfrak{n}$ and $\tau(n)=0$ for all $n \in \mathfrak{n}$. Also observe that $\tau(x) y=0$ for all $x \in \mathfrak{g}$ and $y \in \mathfrak{m} \oplus \mathfrak{v}$.

We will apply Lemma 10.6 of the Appendix to obtain a Lie group analogue of (2.4). Let $\operatorname{Aut}\left(\mathfrak{g}_{N}\right), \operatorname{Aut}\left(G_{N}\right)$ denote the groups of automorphisms of $\mathfrak{g}_{N}$ and $G_{N}$ respectively. Since $G_{N}$ is simply connected $\tau$ determines a homomorphism $\bar{T}: G_{N} \rightarrow \operatorname{Aut}\left(\mathfrak{g}_{N}\right)$ such that $\bar{T}\left(\exp _{G_{N}} x\right) y=e^{\tau(x)} y$ for all $x, y \in \mathfrak{g}_{N}$. Again by simple connectedness, $\operatorname{Aut}\left(\mathfrak{g}_{N}\right)$ is isomorphic to $\operatorname{Aut}\left(G_{N}\right)$ and $\bar{T}$ determines a homomorphism $T: G_{N} \rightarrow \operatorname{Aut}\left(G_{N}\right)$ such that $T(g)\left(\exp _{G_{N}} y\right)=\exp _{G_{N}}(\bar{T}(g)(y))$ for all $g \in G_{N}$ and $y \in \mathfrak{g}_{N}$. For any $x, y, z \in \mathfrak{g}$ we calculate

$$
\begin{aligned}
T\left(T\left(\exp _{G_{N}} x\right) \exp _{G_{N}} y\right)\left(\exp _{G_{N}} z\right) & =\exp _{G_{N}}\left(e^{\tau\left(e^{\tau(x)} y\right)} z\right) \\
& =\exp _{G_{N}}\left(e^{\tau(y)} z\right) \\
& =T\left(\exp _{G_{N}} y\right)\left(\exp _{G_{N}} z\right)
\end{aligned}
$$

where in the second line we have used that $\tau(\tau(x) w)=0$ for all $x, w \in \mathfrak{g}$. 
Therefore $T$ verifies the hypothesis $T(T(g) h)=T(h), g, h \in G_{N}$, of Lemma 10.6 of the Appendix. Applying that lemma with $A=G_{N}$ we obtain a Lie group $G^{\prime}=\left(G_{N}, *\right)$ with underlying manifold $G_{N}$ and group multiplication $*$ given by

$$
g * h=T\left(h^{-1_{N}}\right) g *_{N} h
$$

for all $g, h \in G_{N}$; the identity element of $G^{\prime}$ is the identity $e$ of $G_{N}$, and the Lie algebra $\mathfrak{g}^{\prime}$ of $G^{\prime}$ is related to $\mathfrak{g}_{N}$ by (2.4) (after the identification $\mathfrak{g}^{\prime}=T_{e} G_{N}=\mathfrak{g}_{N}$ as vector spaces).

Therefore $G^{\prime}$ is Lie isomorphic to $G$, because $G, G^{\prime}$ are simply connected Lie groups with isomorphic Lie algebras. In the sequel we identify $G=G^{\prime}$. Thus $G=G_{N}$ as manifolds, with multiplications related by (2.5) and Lie brackets by (2.4). Often we abbreviate $g * h=g h, g, h \in G$.

It follows from Lemma 10.6(IV) that $\bar{T}$ and $T$ are also homomorphisms of $G$. For example

$$
\bar{T}(g h)=\bar{T}(g) \bar{T}(h)=\bar{T}\left(g *_{N} h\right)
$$

for all $g, h \in G$.

For the next lemma we note that $\tau(\mathfrak{g})$ is a subalgebra of the Lie algebra $\mathcal{L}(\mathfrak{g})$ of linear transformations. The kernel of $\tau$ is the largest nilcompact ideal $\mathfrak{n}^{\prime}$ of $\mathfrak{g}$ :

Lemma 2.4 One has $\tau(\mathfrak{g})=\tau(\mathfrak{m}) \oplus \tau(\mathfrak{v})$ with $\tau(\mathfrak{m})$, $\tau(\mathfrak{v})$ mutually commuting ideals of $\tau(\mathfrak{g})$. Moreover

$$
\mathfrak{n}^{\prime}=\{x \in \mathfrak{g}: \tau(x)=0\} .
$$

Proof. Since $\mathfrak{g}=\mathfrak{m} \oplus \mathfrak{v} \oplus \mathfrak{n}$ and $\tau(\mathfrak{n})=\{0\}$ we have $\tau(\mathfrak{g})=\tau(\mathfrak{m})+\tau(\mathfrak{v})$. Since $[\mathfrak{m}, \mathfrak{v}]=\{0\}$ it follows that $\tau(\mathfrak{m}), \tau(\mathfrak{v})$ are mutually commuting ideals in the Lie algebra $\tau(\mathfrak{g})$. Moreover $\tau(\mathfrak{m})$ is semisimple since $\mathfrak{m}$ is semisimple, while $\tau(\mathfrak{v})=\{S(v): v \in \mathfrak{v}\}$ is abelian by Lemma 2.2. Therefore $\tau(\mathfrak{m}) \cap$ $\tau(\mathfrak{v})=\{0\}$ and $\tau(\mathfrak{g})=\tau(\mathfrak{m}) \oplus \tau(\mathfrak{v})$. From this direct sum decomposition and $\tau(\mathfrak{n})=\{0\}$ we get

$$
\begin{aligned}
\operatorname{ker}(\tau) & =(\operatorname{ker}(\tau) \cap \mathfrak{m}) \oplus(\operatorname{ker}(\tau) \cap \mathfrak{v}) \oplus \mathfrak{n} \\
& =\{x \in \mathfrak{m}:[x, \mathfrak{q}]=\{0\}\} \oplus\{v \in \mathfrak{v}: S(v)=0\} \oplus \mathfrak{n} \\
& =\mathfrak{s} \oplus(\mathfrak{v} \cap \mathfrak{n}) \oplus \mathfrak{n}=\mathfrak{s} \oplus \mathfrak{n}=\mathfrak{n}^{\prime},
\end{aligned}
$$

where $\mathfrak{s}=\{x \in \mathfrak{m}:[x, \mathfrak{q}]=\{0\}\}$ by Lemma 10.4 of the Appendix.

By this lemma, $\mathfrak{n}^{\prime}$ is an ideal of both $\mathfrak{g}$ and $\mathfrak{g}_{N}$. From $\tau\left(\mathfrak{n}^{\prime}\right)=\{0\}$ and (2.4) we also see that $[x, y]=[x, y]_{N}$ for all $x, y \in \mathfrak{n}^{\prime}$. 
Let $N^{\prime}=\exp _{G_{N}}\left(\mathfrak{n}^{\prime}\right)$ denote the normal analytic subgroup of $G_{N}$ with Lie algebra $\mathfrak{n}^{\prime}$. It easily follows from Lemma 2.4 that

$$
\bar{T}(n) x=x \quad, \quad T(n) g=g
$$

for all $n \in N^{\prime}, x \in \mathfrak{g}$ and $g \in G$. Therefore (see Lemma 10.6 of the Appendix)

$$
n_{1} * n_{2}^{-1}=T\left(n_{2}\right)\left(n_{1} *_{N} n_{2}^{-1_{N}}\right)=n_{1} *_{N} n_{2}^{-1_{N}}
$$

for all $n_{1}, n_{2} \in N^{\prime}$, so that the group operations of $G$ and $G_{N}$ coincide on $N^{\prime}$. Thus $N^{\prime}$ is also a subgroup of $G$. Since its Lie algebra is the ideal $\mathfrak{n}^{\prime}$ of $\mathfrak{g}$, it is a normal subgroup of $G$.

\subsection{Decomposition of the Lie algebra}

We produce a useful decomposition of $\mathfrak{g}$ into $\tau$-invariant subspaces, following Proposition 2.3 of [1]. The representation $\tau$ is completely reducible by the criterion at the beginning of Section 2, since $\tau(y)$ is a semisimple transformation for all $y$ in the radical $\mathfrak{q}_{N}$ of $\mathfrak{g}_{N}$.

Lemma 2.5 There exist $\tau$-invariant subspaces $\mathfrak{h}_{j}, j \in \mathbb{N}$, of $\mathfrak{q}$ such that $\mathfrak{h}_{1}=\mathfrak{v} \oplus\left(\mathfrak{h}_{1} \cap \mathfrak{n}\right)$ and $\mathfrak{q}_{N ; j}=\mathfrak{h}_{j} \oplus \mathfrak{q}_{N ; j+1}$ for all $j \in \mathbb{N}$.

Proof. Since the subspaces $\mathfrak{q}_{N ; j}$ and $\mathfrak{n}$ are $\tau$-invariant, complete reducibility implies the existence of $\tau$-invariant subspaces $\mathfrak{k}_{1}, \mathfrak{h}_{j}, j \geq 2$, with $\mathfrak{n}=\mathfrak{k}_{1} \oplus \mathfrak{q}_{N ; 2}$ and $\mathfrak{q}_{N ; j}=\mathfrak{h}_{j} \oplus \mathfrak{q}_{N ; j+1}, j \geq 2$. Setting $\mathfrak{h}_{1}=\mathfrak{v} \oplus \mathfrak{k}_{1}$, we have $\mathfrak{k}_{1}=\mathfrak{h}_{1} \cap \mathfrak{n}$ and $\mathfrak{q}=\mathfrak{q}_{N ; 1}=\mathfrak{h}_{1} \oplus \mathfrak{q}_{N ; 2}$.

If subspaces $\mathfrak{h}_{j}$ are chosen as in the lemma, then $\mathfrak{h}_{1}$ generates the Lie algebra $\mathfrak{q}_{N}$ by Proposition 2.3, and setting $\mathfrak{k}_{1}=\mathfrak{h}_{1} \cap \mathfrak{n}$ we obtain decompositions

$$
\mathfrak{q}=\mathfrak{h}_{1} \oplus \mathfrak{h}_{2} \oplus \cdots \oplus \mathfrak{h}_{r} \quad, \quad \mathfrak{n}=\mathfrak{k}_{1} \oplus \mathfrak{q}_{N ; 2}=\mathfrak{k}_{1} \oplus \mathfrak{h}_{2} \oplus \cdots \oplus \mathfrak{h}_{r}
$$

and

$$
\mathfrak{g}=\mathfrak{m} \oplus \mathfrak{v} \oplus \mathfrak{k}_{1} \oplus \mathfrak{h}_{2} \oplus \cdots \oplus \mathfrak{h}_{r}
$$

into $\tau$-invariant subspaces.

Note that $\tau$ is determined by the pair $(\mathfrak{m}, \mathfrak{v})$ satisfying the properties of Lemma 2.1. Significant simplifications in analysis on $G$ occur in the following case: 
Definition 2.6 We say that $\mathfrak{g}$ has stratified nilshadow with respect to $(\mathfrak{m}, \mathfrak{v})$ if there exist subspaces $\mathfrak{h}_{j}$ satisfying all properties of Lemma 2.5 and in addition

$$
\left[\mathfrak{h}_{j}, \mathfrak{h}_{k}\right]_{N} \subseteq \mathfrak{h}_{j+k}
$$

for all $j, k \in \mathbb{N}$. In this case we say that $G$ has stratified nilshadow.

If Definition 2.6 is satisfied, then the nilshadow $\mathfrak{q}_{N}$ is a stratified nilpotent Lie algebra with stratification $\left\{\mathfrak{h}_{j}\right\}_{j \in \mathbb{N}}$, in the usual sense (see [25]). Note our definition requires that the stratification $\left\{\mathfrak{h}_{j}\right\}$ be appropriately compatible with $(\mathfrak{m}, \mathfrak{v})$.

In the sequel we fix $\mathfrak{h}_{j}$ satisfying all properties of Lemma 2.5.

For the next result the type $R$ property of $\mathfrak{g}$ is essential.

Lemma 2.7 There exists a positive-definite inner product $(\cdot, \cdot)$ on $\mathfrak{g}$ such that all subspaces in the decomposition (2.7) are mutually orthogonal, and

$$
(\tau(x) a, b)=-(a, \tau(x) b) \quad, \quad(\bar{T}(g) a, \bar{T}(g) b)=(a, b),
$$

for all $a, b, x \in \mathfrak{g}, g \in G$.

Proof. It suffices to prove that any $\tau$-invariant subspace $U$ of $\mathfrak{g}$ has an inner product $(\cdot, \cdot)_{U}$ such that $(\tau(x) a, b)_{U}=-(a, \tau(x) b)_{U}$ for all $x \in \mathfrak{g}$, $a, b \in U$; then apply this result to each subspace in $(2.7)$ and let $(\cdot, \cdot)$ be the orthogonal direct sum of the subspace inner products.

Thus let $U \subseteq \mathfrak{g}$ be $\tau$-invariant. The complexifications $\tau(v)^{\mathbb{C}}=S(v)^{\mathbb{C}}$, $v \in \mathfrak{v}$, are mutually commuting, semisimple transformations of $U_{\mathbb{C}}$ with only purely imaginary eigenvalues. Hence there is a basis $u_{1}, \ldots, u_{s}$ of $U_{\mathbb{C}}$ and linear maps $\lambda_{1}, \ldots, \lambda_{s}: \mathfrak{v} \rightarrow \mathbb{R}$ such that

$$
\tau(v)^{\mathbb{C}} u_{j}=i \lambda_{j}(v) u_{j}
$$

for all $v \in \mathfrak{v}, j \in\{1, \ldots, s\}$. If $(\cdot, \cdot)_{1}$ is the inner product on $U_{\mathbb{C}}$ such that the basis $u_{1}, \ldots, u_{s}$ is orthonormal, then $(\tau(v) a, b)_{1}=-(a, \tau(v) b)_{1}, a, b \in U$, $v \in \mathfrak{v}$. For $a, b \in U$ define

$$
(a, b)_{U}=\int_{M} d m(\bar{T}(m) a, \bar{T}(m) b)_{1}
$$

where $d m$ is normalized Haar measure on the compact group $M$. Then $(\cdot, \cdot)_{U}$ is an inner product such that $(\tau(x) a, b)_{U}=-(a, \tau(x) b)_{U}$ for all $x \in \mathfrak{m}$. One has $(\tau(v) a, b)_{U}=-(a, \tau(v) b)_{U}$ for $v \in \mathfrak{v}$, because $[\mathfrak{m}, \mathfrak{v}]=\{0\}$ implies that $\tau(v)$ commutes with $\bar{T}(m), m \in M$. Since $\tau(\mathfrak{g})=\tau(\mathfrak{m})+\tau(\mathfrak{v})$ by Lemma 2.4, the lemma is proved.

Henceforth we fix the inner product $(\cdot, \cdot)$ as in Lemma 2.7 . 
Corollary 2.8 Let $d g$ denote Haar measure for the group $G_{N}$. The transformations $T(g), g \in G_{N}$, preserve $d g$ in the sense that $d g(T(g) A)=d g(A)$ for Borel measurable $A \subseteq G_{N}$, and $d g$ is Haar measure for $G$.

Proof. One has $d g=d m \times d q$ where $d m, d q$ are Haar measures for $M$, $Q_{N}$ respectively. By Lemma 2.7 the restriction $\left.\bar{T}(g)\right|_{\mathfrak{q}_{N}}$ is an orthogonal transformation of $\mathfrak{q}_{N}$ with respect to $(\cdot, \cdot)$, hence it preserves Lebesgue measure on $\mathfrak{q}_{N}$. Since $Q_{N}$ is simply connected nilpotent, $\exp _{Q_{N}}: \mathfrak{q}_{N} \rightarrow Q_{N}$ is a diffeomorphism mapping Lebesgue measure to $d q$. Therefore the restriction $\left.T(g)\right|_{Q_{N}}$ preserves $d q$, and since $T(g) m=m, m \in M$, it follows that $T(g)$ preserves $d g$.

If $\varphi \in C_{c}(G)$ then applying (2.5) gives

$$
\begin{aligned}
\int d g \varphi(g h) & =\int d g \varphi\left(T\left(h^{-1_{N}}\right) g *_{N} h\right) \\
& =\int d g \varphi\left(T\left(h^{-1_{N}}\right) g\right)=\int d g \varphi(g)
\end{aligned}
$$

for all $h \in G_{N}$. Therefore $d g$ is Haar measure for $G$.

In the sequel we fix the measure $d g$ and consider the spaces $L_{p}=$ $L_{p}\left(G_{N} ; d g\right)=L_{p}(G ; d g), 1 \leq p \leq \infty$.

Finally, from the tools of this subsection we derive another characterization of $\mathfrak{n}^{\prime}$ which is needed for the proof of Theorem 1.6.

Lemma 2.9 Let $x \in \mathfrak{g}$. Then $x \in \mathfrak{n}^{\prime}$ if and only if the transformation $\operatorname{ad}_{\mathfrak{q}} x \in \mathcal{L}(\mathfrak{q})$ is nilpotent. If $x \notin \mathfrak{n}^{\prime}$, there exists $n \in \mathfrak{n}$ such that $(\operatorname{ad} x)^{j} n \notin$ $\mathfrak{q}_{N ; 2}$ for all $j \in \mathbb{N}$.

Remark Since $\mathfrak{n}, \mathfrak{q}_{N ; 2}$ are ideals of $\mathfrak{g}$, it is clear that $n \notin \mathfrak{q}_{N ; 2}$ and $(\operatorname{ad} x)^{j} n \in$ $\mathfrak{n}$ for all $j \in \mathbb{N}$.

Proof. If $x \in \mathfrak{n}^{\prime}$ then $x=m+y, m \in \mathfrak{s}, y \in \mathfrak{n}$, and since $[\mathfrak{s}, \mathfrak{q}]=\{0\}$ we have $\operatorname{ad}_{\mathfrak{q}} x=\operatorname{ad}_{\mathfrak{q}} y=K_{\mathfrak{q}}(y)$. Hence $\operatorname{ad}_{\mathfrak{q}} x$ is nilpotent.

Suppose that $x \notin \mathfrak{n}^{\prime}$. We claim that $\tau(x)\left(\mathfrak{k}_{1}\right) \neq\{0\}$. Otherwise, if $\tau(x)\left(\mathfrak{k}_{1}\right)=\{0\}$ then $\tau(x)\left(\mathfrak{h}_{1}\right)=\{0\}$, because $\mathfrak{h}_{1}=\mathfrak{v} \oplus \mathfrak{k}_{1}$ and $\tau(y)(\mathfrak{v})=\{0\}$ for all $y \in \mathfrak{g}$. Since $\mathfrak{h}_{1}$ generates $\mathfrak{q}_{N}$ and $\tau(x)$ is a derivation of $\mathfrak{q}_{N}$ it follows that $\tau(x)\left(\mathfrak{q}_{N}\right)=\{0\}$. But $\tau(y)(\mathfrak{m})=\{0\}$ for all $y \in \mathfrak{g}$. Thus $\tau(x)=0$, which contradicts Lemma 2.4 and proves the claim.

Therefore we choose $n \in \mathfrak{k}_{1} \subseteq \mathfrak{n}$ with $\tau(x) n \neq 0$. By Lemma 2.7, $\tau(x)$ is a skew-symmetric, hence a semisimple transformation. Therefore $\tau(x)^{j} n \neq 0$ for all $j \in \mathbb{N}$. Since $\mathfrak{k}_{1}$ is $\tau$-invariant and $\mathfrak{k}_{1} \cap \mathfrak{q}_{N ; 2}=\{0\}$, it follows that

$$
\tau(x)^{j} n \notin \mathfrak{q}_{N ; 2}
$$

for all $j \in \mathbb{N}$. 
From $(2.4)$ and $\tau(\mathfrak{n})=\{0\}$ we have $[x, n]=[x, n]_{N}+\tau(x) n$. But $[x, n]_{N} \in$ $\left[\mathfrak{g}_{N}, \mathfrak{n}\right]_{N} \subseteq \mathfrak{q}_{N ; 2}$ so that

$$
(\operatorname{ad} x) n-\tau(x) n \in \mathfrak{q}_{N ; 2}
$$

Repetition of this argument (using the $\tau$-invariance and ad-invariance of $\mathfrak{n}$ and $\mathfrak{q}_{N ; 2}$ ) yields

$$
(\operatorname{ad} x)^{j} n-\tau(x)^{j} n \in \mathfrak{q}_{N ; 2}
$$

for all $j \in \mathbb{N}$. Therefore $(\operatorname{ad} x)^{j} n \notin \mathfrak{q}_{N ; 2}$ for all $j \in \mathbb{N}$. In particular $\operatorname{ad}_{\mathfrak{q}} x$ is not nilpotent, and the proof of the lemma is complete.

\subsection{Derivatives and distances}

In this subsection we compare derivatives and distances on the groups $G$ and $G_{N}$.

First we define a space of coefficient functions. For $a, b \in \mathfrak{g}$ define $\Lambda_{a, b}: G \rightarrow \mathbb{R}$ by

$$
\Lambda_{a, b}(g)=(\bar{T}(g) a, b)=(b, \bar{T}(g) a)
$$

for $g \in G$, and let $\mathcal{E}_{1}$ denote the complex vector space spanned by all $\Lambda_{a, b}$, $a, b \in \mathfrak{g}$.

Lemma $2.10 \mathcal{E}_{1}$ is a finite-dimensional vector space of smooth bounded functions on $G$ which contains the constant functions.

$\mathcal{E}_{1}$ is invariant under the left or right regular representations of $G_{N}$ or $G$ acting in $L_{\infty}$, that is, $L_{G_{N}}, L_{G}, R_{G_{N}}, R_{G}$ map $\mathcal{E}_{1}$ into itself.

If $\sigma \in \mathcal{E}_{1}$ then $d L_{G}(x)(\sigma)=d L_{G_{N}}(x)(\sigma) \in \mathcal{E}_{1}$, and $\check{\sigma} \in \mathcal{E}_{1}$ where $\check{\sigma}(g)=$ $\sigma\left(g^{-1}\right)=\sigma\left(g^{-1_{N}}\right), g \in G$.

Proof. Since $\bar{T}(g)$ is orthogonal with respect to the inner product $(\cdot, \cdot)$, one has $\Lambda_{a, b} \in L_{\infty}$. The statements about invariance under the regular representations are standard consequences of the fact that $\bar{T}$ is a representation of $G$, and $G_{N}$, by orthogonal transformations (cf. [24,6]). For example, noting that

$$
\left(\bar{T}\left(h^{-1} g\right) a, b\right)=(\bar{T}(g) a, \bar{T}(h) b)=\left(\bar{T}\left(h^{-1_{N}} g\right) a, b\right)
$$

establishes that

$$
L_{G}(h) \Lambda_{a, b}=L_{G_{N}}(h) \Lambda_{a, b}=\Lambda_{a, \bar{T}(h) b} \in \mathcal{E}_{1} .
$$

If $x \in \mathfrak{g}$, choose a curve $\gamma:[-1,1] \rightarrow G$ with $\dot{\gamma}(0)=x$. Setting $h=\gamma(t)$ in the above relation and differentiating at $t=0$ yields $d L_{G}(x)\left(\Lambda_{a, b}\right)=$ $d L_{G_{N}}(x)\left(\Lambda_{a, b}\right)=\Lambda_{a, \tau(x) b}$. 
Similarly one sees that $\Lambda_{a, b}\left(g^{-1}\right)=\Lambda_{a, b}\left(g^{-1_{N}}\right)=\Lambda_{b, a}(g), g \in G$.

If $\operatorname{dim}(\mathfrak{m} \oplus \mathfrak{v})>0$ pick a nonzero $a \in \mathfrak{m} \oplus \mathfrak{v}$, otherwise $\mathfrak{g}=\mathfrak{n}$ and we pick any nonzero $a \in \mathfrak{g}$. Then $\tau(x) a=0$ for all $x \in \mathfrak{g}$, hence $\bar{T}(g) a=a$ for all $g \in G$, and therefore $\Lambda_{a, a}$ is a nonzero constant function on $G$. The lemma is proved.

Remark Elements of $\mathcal{E}_{1}$ are automatically almost-periodic functions on $G$, because they are matrix elements of the bounded, finite-dimensional representation $\bar{T}$ (see for example [10, Chapter 7$]$ ). The almost-periodicity is important for homogenization theory on $G$ (cf. [1, 2]) but is not used in this paper.

Lemma 2.11 Functions in $\mathcal{E}_{1}$ are constant along cosets of $N^{\prime}$ in the sense that

$$
\sigma(g * n)=\sigma(n * g)=\sigma\left(g *_{N} n\right)=\sigma\left(n *_{N} g\right)=\sigma(g)
$$

for all $\sigma \in \mathcal{E}_{1}, g \in G$ and $n \in N^{\prime}$.

Proof. Since $\bar{T}$ is a representation of both $G$ and $G_{N}$ this is immediate from (2.6).

To compare derivatives on $G$ and $G_{N}$ it is useful to introduce a basis. Let $p=\operatorname{dim}(\mathfrak{m})-1 \in\{-1,0,1,2, \ldots\}, d=\operatorname{dim}(\mathfrak{q}), d_{0}=\operatorname{dim}(\mathfrak{q} / \mathfrak{n})=\operatorname{dim}(\mathfrak{v})$, and $d_{1}=\operatorname{dim}\left(\mathfrak{q} / \mathfrak{q}_{N ; 2}\right)=\operatorname{dim}\left(\mathfrak{h}_{1}\right)$. Fix an orthonormal basis

$$
b_{-p}, \ldots, b_{0}, b_{1}, \ldots, b_{d}
$$

for $\mathfrak{g}$ which respects the orthogonal decomposition (2.7). In particular $b_{-p}, \ldots, b_{0}$ is a basis of $\mathfrak{m}, b_{1}, \ldots, b_{d_{0}}$ is a basis of $\mathfrak{v}, b_{1}, \ldots, b_{d_{1}}$ is a basis of $\mathfrak{h}_{1}$, and $b_{d_{0}+1}, \ldots, b_{d}$ is a basis of $\mathfrak{n}$.

We assign weights $w(j) \in\{0,1, \ldots, r\}$ to the $b_{j}$ such that $w(j)=0$ for $j \in\{-p, \ldots, 0\}$ and $b_{j} \in \mathfrak{h}_{w(j)}$ for $j \in\{1, \ldots, d\}$. Then

$$
\mathfrak{h}_{s}=\operatorname{span}\left\{b_{j}: w(j)=s\right\} \quad, \quad \mathfrak{q}_{N ; s}=\operatorname{span}\left\{b_{j}: w(j) \geq s\right\} \quad,
$$

for all $s \in\{1, \ldots, r\}$. For $j \in\{-p, \ldots, d\}$ we set $B_{j}=d L_{G}\left(b_{j}\right)$ and $B_{j}^{(N)}=$ $d L_{G_{N}}\left(b_{j}\right)$.

For derivatives we use multi-index notations as follows. If $h, k$ are integers with $h \leq k$ set

$$
J(h, k)=\bigcup_{n=0}^{\infty}\{h, h+1, \ldots, k\}^{n}
$$

and when $k \geq 1$ let $J(k)=J(1, k)$. If $\alpha=\left(i_{1}, \ldots, i_{n}\right) \in J(h, k)$ then the length of $\alpha$ is defined by $|\alpha|=n$ (then $|\alpha|=0$ if $\alpha$ is the empty multi-index). If $\alpha=\left(i_{1}, \ldots, i_{n}\right) \in J\left(d^{\prime}\right)$ set $A^{\alpha}=A_{i_{1}} \ldots A_{i_{n}}$ where $A_{i}=d L_{G}\left(a_{i}\right)$, and set $a_{[\alpha]}=\left[a_{i_{1}},\left[a_{i_{2}}, \cdots\left[a_{i_{n-1}}, a_{i_{n}}\right] \cdots\right]\right]$. 
Similarly if $\alpha=\left(i_{1}, \ldots, i_{n}\right) \in J(-p, d)$ we set

$$
B^{\alpha}=B_{i_{1}} \ldots B_{i_{n}} \quad, \quad B^{(N) \alpha}=B_{i_{1}}^{(N)} \ldots B_{i_{n}}^{(N)},
$$

and define the weighted length

$$
\|\alpha\|=w\left(i_{1}\right)+\cdots+w\left(i_{n}\right) \in \mathbb{N}_{0} .
$$

We write $J(\mathfrak{n})=J\left(d_{0}+1, d\right)$. Then for $\alpha \in J(\mathfrak{n})$ the derivatives $B^{\alpha}, B^{(N) \alpha}$ are in the direction of $\mathfrak{n}$.

Lemma 2.12 If $a \in \mathfrak{g}, \varphi \in L_{2 ; \infty}$ then

$$
\left(d L_{G}(a) \varphi\right)(g)=\left(d L_{G_{N}}\left(\bar{T}\left(g^{-1_{N}}\right) a\right) \varphi\right)(g)
$$

for all $g \in G$ and

$$
d L_{G}(a) \varphi=\sum_{j=-p}^{d} \sigma_{j} B_{j}^{(N)} \varphi
$$

where $\sigma_{j}=\Lambda_{b_{j}, a} \in \mathcal{E}_{1}$. The $\sigma_{j}$ are constants for $j \in\left\{-p, \ldots, d_{0}\right\}$. Moreover

$$
d L_{G_{N}}(a) \varphi=\sum_{j=-p}^{d} \check{\sigma}_{j} B_{j} \varphi
$$

with $\check{\sigma}_{j}=\Lambda_{a, b_{j}} \in \mathcal{E}_{1}$.

Proof. The first equation is a consequence of Lemma 10.6 applied to the groups $A=G_{N}, A^{\prime}=G$. Observing that

$$
\bar{T}\left(g^{-1_{N}}\right) a=\sum_{j=-p}^{d}\left(\bar{T}\left(g^{-1_{N}}\right) a, b_{j}\right) b_{j}=\sum_{j=-p}^{d} \Lambda_{b_{j}, a}(g) b_{j}
$$

gives the second equation. Since $\bar{T}(g) y=y$ for all $y \in \mathfrak{m} \oplus \mathfrak{v}$ it follows that $\Lambda_{b_{j}, a}=\left(b_{j}, a\right)$ is constant when $j \leq d_{0}$.

Writing $a=\bar{T}\left(g^{-1_{N}}\right) \bar{T}(g) a$, we deduce from the first equation of the lemma that $\left(d L_{G_{N}}(a) \varphi\right)(g)=\left(d L_{G}(\bar{T}(g) a) \varphi\right)(g)$. Since

$$
\bar{T}(g) a=\sum_{j} \Lambda_{a, b_{j}}(g) b_{j},
$$

we get the desired expression for $d L_{G_{N}}(a)$.

Let $\mathcal{E}$ denote the subalgebra of $L_{\infty}$ generated by $\mathcal{E}_{1}$; it is spanned by all products $\sigma_{1} \cdots \sigma_{k}, k \in \mathbb{N}, \sigma_{1}, \ldots, \sigma_{k} \in \mathcal{E}_{1}$.

Let $\mathcal{R}(G), \mathcal{R}\left(G_{N}\right)$ denote the algebras of complex right-invariant differential operators on $G$ and $G_{N}$ respectively. Then $\mathcal{R}(G)$ (respectively $\mathcal{R}\left(G_{N}\right)$ ) is spanned by $\left\{B^{\alpha}, \alpha \in J(-p, d)\right\}$ (respectively $\left\{B^{(N) \alpha}, \alpha \in J(-p, d)\right\}$ ). By combining the last three lemmas we obtain 
Corollary 2.13 The subalgebra $\mathcal{E} \subseteq L_{\infty}$ is invariant under the left and right regular representations of $G$ and $G_{N}$, and $d L_{G}(x) \sigma=d L_{G_{N}}(x) \sigma \in \mathcal{E}$ whenever $\sigma \in \mathcal{E}, x \in \mathfrak{g}$. Each $\sigma \in \mathcal{E}$ is constant along cosets of $N^{\prime}$ in the sense of Lemma 2.11.

Moreover $\mathcal{R}(G)$ (respectively $\mathcal{R}\left(G_{N}\right)$ ) is contained in the span of all differential operators of the form $\sigma P, \sigma \in \mathcal{E}, P \in \mathcal{R}\left(G_{N}\right)$ (respectively $P \in \mathcal{R}(G))$.

Therefore $L_{2 ; \infty}=L_{2 ; \infty}\left(G_{N}\right)$, that is, the $C^{\infty}$ subspaces of $L_{2}$ for the representations $L_{G}, L_{G_{N}}$ coincide.

Next we compare various distances. Recall that $g \mapsto|g|_{a}$ denotes the modulus on $G$ associated with $a_{1}, \ldots, a_{d^{\prime}}$. Thus

$$
|g|_{a}=\inf _{\gamma} \int_{0}^{1} d t\left(\sum_{k=1}^{d^{\prime}} \xi_{k}(t)^{2}\right)^{1 / 2}
$$

with the infimum over all absolutely continuous paths $\gamma:[0,1] \rightarrow G$ with

$$
\gamma(0)=e, \gamma(1)=g \quad \text { and } \quad \dot{\gamma}(t)=\left.\sum_{k=1}^{d^{\prime}} \xi_{k}(t) A_{k}\right|_{\gamma(t)}
$$

(cf. [30], [35]). Similarly, let $g \mapsto|g|$ denote the modulus on $G$ associated with $b_{-p}, \ldots, b_{d_{1}}, g \mapsto|g|_{N}$ the modulus on $G_{N}$ associated with $b_{-p}, \ldots, b_{d_{1}}$, $m \mapsto|m|_{M}$ the modulus on $M$ associated with $b_{-p}, \ldots, b_{0}$, and $q \mapsto|q|_{Q_{N}}$ the modulus on $Q_{N}$ associated with $b_{1}, \ldots, b_{d_{1}}$.

Lemma 2.14 (I) For each compact neighborhood $U$ of e there exists $c=$ $c(U)>0$ with

$$
c^{-1}|g| \leq|g|_{a} \leq c|g|
$$

for all $g \in G$ with $g \notin U$.

(II) $|g|^{2}=|g|_{N}^{2}=|m|_{M}^{2}+|q|_{Q_{N}}^{2}$ for all $g=m *_{N} q \in G$ with $m \in M$, $q \in Q_{N}$.

(III) The volume function $V_{a}(t)=d g\left\{g \in G:|g|_{a}<t\right\}$ satisfies

$$
c^{-1} t^{D} \leq V_{a}(t) \leq c t^{D}
$$

for all $t \geq 1$, where

$$
D=\sum_{k=1}^{r} k \operatorname{dim}\left(\mathfrak{q}_{N ; k} / \mathfrak{q}_{N ; k+1}\right)=\sum_{j=1}^{d} w(j)
$$

(IV) $|T(h) g|=|g|$ and $\left|g * h^{-1}\right|=\left|g *_{N} h^{-1_{N}}\right|$ for all $g, h \in G$. 
Proof. For (I), see [35, Proposition III.4.2]. For (II), note from Lemma 2.12 that

$$
\left.B_{i}^{(N)}\right|_{g}=\left.\sum_{j=-p}^{d_{1}} T_{i, j}(g) B_{j}\right|_{g}
$$

for all $g \in G$ and $i \in\left\{-p, \ldots, d_{1}\right\}$, where $T_{i, j}=\Lambda_{b_{i}, b_{j}}$. The matrix $\left(T_{i, j}(g)\right)$ is orthogonal. Hence if $\gamma$ is a path with

$$
\dot{\gamma}(t)=\left.\sum_{i=-p}^{d_{1}} \xi_{i}(t) B_{i}^{(N)}\right|_{\gamma(t)},
$$

then $\dot{\gamma}(t)=\left.\sum_{i=-p}^{d_{1}} \eta_{i}(t) B_{i}\right|_{\gamma(t)}$ where $\sum_{i} \xi_{i}(t)^{2}=\sum_{i} \eta_{i}(t)^{2}$. Therefore $|g| \leq$ $|g|_{N}$, and the reverse inequality is proved similarly. The straightforward proof that $|g|_{N}^{2}=|m|_{M}^{2}+|q|_{Q_{N}}^{2}$ is left to the reader.

Since $Q_{N}$ is simply connected nilpotent, from [35, Section IV.5] we have

$$
c^{-1} t^{D} \leq d q\left\{q \in Q_{N}:|q|_{Q_{N}}<t\right\} \leq c t^{D}
$$

for $t \geq 1$. Then (III) follows easily from (I), (II) and the compactness of $M$.

If $\gamma$ is a path as above, then the path $\gamma_{h}=T(h) \circ \gamma$ satisfies

$$
\dot{\gamma}_{h}(t)=\left.\sum_{j=-p}^{d_{1}} \rho_{j}(t) B_{j}^{(N)}\right|_{\gamma_{h}(t)},
$$

where $\rho_{j}(t)=\sum_{i=-p}^{d_{1}} T_{i, j}(h) \xi_{i}(t)$. Then $\sum_{i} \rho_{i}(t)^{2}=\sum_{i} \xi_{i}(t)^{2}$ and we obtain $|T(h) g| \leq|g|$. But $|g|=\left|T\left(h^{-1_{N}}\right) T(h) g\right| \leq|T(h) g|$ so $|T(h) g|=|g|$.

Finally, from Lemma 10.6 we have $g * h^{-1}=T(h)\left(g *_{N} h^{-1_{N}}\right)$ and the last statement in (IV) follows.

\subsection{Stratified nilshadows and scaling}

In this subsection, we study scaling on $G$ under the assumption that $\mathfrak{g}$ has stratified nilshadow. That is, we assume that (2.8) holds.

Then the dilations $\gamma_{\varepsilon} \in \mathcal{L}\left(\mathfrak{q}_{N}\right)$ defined by $\gamma_{\varepsilon}\left(x_{j}\right)=\varepsilon^{j} x_{j}$ for $\varepsilon>0, x_{j} \in \mathfrak{h}_{j}$, $j \in\{1, \ldots, r\}$, are automorphisms of $\mathfrak{q}_{N}$. They extend to automorphisms of $\mathfrak{g}_{N}$ by setting $\gamma_{\varepsilon}(x+y)=x+\gamma_{\varepsilon}(y)$ for $x \in \mathfrak{m}, y \in \mathfrak{q}_{N}$.

Since $G_{N}$ is simply connected there exist automorphisms $\Gamma_{\varepsilon}$ of $G_{N}$ with $\Gamma_{\varepsilon}\left(\exp _{G_{N}} x\right)=\exp _{G_{N}}\left(\gamma_{\varepsilon} x\right)$ for all $x \in \mathfrak{g}$. Then $\Gamma_{\varepsilon} \circ \Gamma_{\delta}=\Gamma_{\varepsilon \delta}$ for $\varepsilon, \delta>0$, $\Gamma_{\varepsilon}\left(Q_{N}\right) \subseteq Q_{N}$, and $Q_{N}$ is a stratified nilpotent group with the dilations $\left.\Gamma_{\varepsilon}\right|_{Q_{N}}$ (see [25]). Note that $\Gamma_{\varepsilon}(m)=m$ for all $m \in M$.

Define $V_{\varepsilon}: L_{2} \rightarrow L_{2}$ by $V_{\varepsilon} \varphi=\varepsilon^{D / 2}\left(\varphi \circ \Gamma_{\varepsilon}\right)$. 
Lemma 2.15 (I) $V_{\varepsilon}: L_{2} \rightarrow L_{2}$ is a unitary map, and $V_{\varepsilon}\left(L_{2 ; \infty}\right) \subseteq L_{2 ; \infty}$ for all $\varepsilon>0$.

(II) For $j \in\{-p, \ldots, d\}, B_{j}^{(N)}$ is homogeneous of order $w(j)$ in the sense that

$$
V_{\varepsilon}^{-1} B_{j}^{(N)} V_{\varepsilon}=\varepsilon^{w(j)} B_{j}^{(N)}
$$

for all $\varepsilon>0$.

(III) $\left|\Gamma_{\varepsilon} q\right|=\varepsilon|q|$ for all $q \in Q_{N}$ and $\varepsilon>0$.

Proof. That $V_{\varepsilon}$ is unitary is equivalent to the statement that $d g\left(\Gamma_{\varepsilon}(A)\right)=$ $\varepsilon^{D} d g(A)$ for Borel measurable $A \subseteq G_{N}$. Since the restriction $\gamma_{\varepsilon} \mid \mathfrak{q}_{N}$ has determinant $\varepsilon^{D}$, this follows by repeating the argument of Corollary 2.8.

Next, since $\Gamma_{\varepsilon}$ is an automorphism of $G_{N}$, one easily sees that $\varphi \circ \Gamma_{\varepsilon} \in$ $L_{2 ; \infty}\left(G_{N}\right)$ whenever $\varphi \in L_{2 ; \infty}\left(G_{N}\right)$. But $L_{2 ; \infty}\left(G_{N}\right)=L_{2 ; \infty}$ by Corollary 2.13, and part (I) follows.

(II) and (III) are straightforward consequences of the definitions and the automorphism property of $\Gamma_{\varepsilon}$; details are left to the reader.

Define $G_{\varepsilon}(\varepsilon>0)$ to be the Lie group with underlying manifold $G=G_{N}$ and multiplication law

$$
g *_{\varepsilon} h=\Gamma_{\varepsilon}\left(\left(\Gamma_{\varepsilon}^{-1} g\right) *\left(\Gamma_{\varepsilon}^{-1} h\right)\right)
$$

for $g, h \in G$. Note that $G_{1}=G$ and that $\Gamma_{\varepsilon}: G \rightarrow G_{\varepsilon}$ is a Lie isomorphism. For the Lie algebra $\mathfrak{g}_{\varepsilon}$ of $G_{\varepsilon}$ one has $\mathfrak{g}_{\varepsilon}=T_{e} G=\mathfrak{g}$ as vector spaces and the Lie bracket is

$$
[x, y]_{\varepsilon}=\gamma_{\varepsilon}\left(\left[\gamma_{\varepsilon}^{-1} x, \gamma_{\varepsilon}^{-1} y\right]\right)
$$

for all $x, y \in \mathfrak{g}$. Since the subspaces $\mathfrak{h}_{j}$ are $\tau$-invariant, one has $\gamma_{\varepsilon} \circ \tau(x)=$ $\tau(x) \circ \gamma_{\varepsilon}$ for $x \in \mathfrak{g}$ and hence $\Gamma_{\varepsilon} \circ T(g)=T(g) \circ \Gamma_{\varepsilon}$ for all $g \in G$ and $\varepsilon>0$. The group multiplications of $G_{\varepsilon}$ and $G_{N}$ are related by

$$
\begin{aligned}
g *_{\varepsilon} h & =\Gamma_{\varepsilon}\left\{\left(T\left(\Gamma_{\varepsilon}^{-1}\left(h^{-1_{N}}\right)\right)\left(\Gamma_{\varepsilon}^{-1} g\right)\right) *_{N}\left(\Gamma_{\varepsilon}^{-1} h\right)\right\} \\
& =\left(\Gamma_{\varepsilon} \circ T\left(\Gamma_{\varepsilon}^{-1}\left(h^{-1_{N}}\right)\right) \circ \Gamma_{\varepsilon}^{-1}\right)(g) *_{N} h=T^{(\varepsilon)}\left(h^{-1_{N}}\right) g *_{N} h
\end{aligned}
$$

where $T^{(\varepsilon)}=T \circ \Gamma_{\varepsilon}^{-1}: G_{N} \rightarrow \operatorname{Aut}\left(G_{N}\right)$. Observe that the argument of Corollary 2.8 shows that $d g$ is a Haar measure for $G_{\varepsilon}$.

Finally, we define rescaled versions of the operator $H$. Set $A_{k, \varepsilon}=$ $d L_{G_{\varepsilon}}\left(\varepsilon^{-1} \gamma_{\varepsilon} a_{k}\right)$ for $k \in\left\{1, \ldots, d^{\prime}\right\}$ and let

$$
H_{\varepsilon}=-\sum_{k, l=1}^{d^{\prime}} c_{k l} A_{k, \varepsilon} A_{l, \varepsilon}
$$


Then $A_{k, \varepsilon}, H_{\varepsilon}$ are right-invariant operators on $G_{\varepsilon}$, and $A_{k, 1}=A_{k}, H_{1}=H$. Because $\Gamma_{\varepsilon}: G \rightarrow G_{\varepsilon}$ and $\gamma_{\varepsilon}: \mathfrak{g} \rightarrow \mathfrak{g}_{\varepsilon}$ are Lie isomorphisms we have

$$
d L_{G_{\varepsilon}}\left(\gamma_{\varepsilon} a_{k}\right) \varphi=\left(d L_{G}\left(a_{k}\right)\left(\varphi \circ \Gamma_{\varepsilon}\right)\right) \circ \Gamma_{\varepsilon}^{-1}=V_{\varepsilon}^{-1} d L_{G}\left(a_{k}\right) V_{\varepsilon} \varphi
$$

for $\varphi$ in the domain of $A_{k, \varepsilon}$. This yields the scaling relations

$$
A_{k, \varepsilon}=\varepsilon^{-1} V_{\varepsilon}^{-1} A_{k} V_{\varepsilon} \quad, \quad H_{\varepsilon}=\varepsilon^{-2} V_{\varepsilon}^{-1} H V_{\varepsilon}
$$

for all $\varepsilon>0$. By Lemma 2.12 we can express

$$
A_{k}=\sum_{j=-p}^{d} \sigma_{k, j} B_{j}^{(N)}
$$

(with equality in the sense of differential operators acting in $L_{2 ; \infty}$ ) where $\sigma_{k, j} \in \mathcal{E}_{1}$, and $\sigma_{k, j}$ constant for $j \in\left\{-p, \ldots, d_{0}\right\}$.

It follows using Lemma 2.15(II) that

$$
A_{k, \varepsilon}=\sum_{j=-p}^{d} \varepsilon^{-1+w(j)} \sigma_{k, j}^{(\varepsilon)} B_{j}^{(N)}
$$

where $\sigma_{k, j}^{(\varepsilon)}=\sigma_{k, j} \circ \Gamma_{\varepsilon}^{-1}$ are bounded functions.

Also, since $\Gamma_{\varepsilon}\left(N^{\prime}\right) \subseteq N^{\prime}$ and from Lemma 2.11 we have

$$
\sigma_{k, j}^{(\varepsilon)}\left(n *_{N} g\right)=\sigma_{k, j}^{(\varepsilon)}\left(g *_{N} n\right)=\sigma_{k, j}^{(\varepsilon)}(g)
$$

for all $n \in N^{\prime}$ and $g \in G$.

Remark If $\mathfrak{g}$ does not have stratified nilshadow, it is still possible to define dilation maps $\gamma_{\varepsilon}, \Gamma_{\varepsilon}$ and the groups $G_{\varepsilon}$. But in general, the $B_{j}^{(N)}$ are not homogeneous in the sense of Lemma 2.15, and equations (2.9), (2.11) are not valid.

One can express $B_{j}^{(N)}$ or $A_{k, \varepsilon}$ in terms of homogeneous vector fields on a group $G_{S}=M \times Q_{S}$, where $Q_{S}$ is a stratified limit group corresponding to the nilpotent group $Q_{N}$ (cf. $\left.[1,29]\right)$. But when $Q_{S} \neq Q_{N}$ the coefficients in this expression involve polynomials and hence may be unbounded. Thus the assumption of stratified nilshadow leads to a simplification in the form of $A_{k, \varepsilon}$ which will be convenient later. 


\section{Preliminary estimates}

In this section we record some initial regularity estimates for the operator $H$ in $L^{2}$, which serve as the basis for the proof of Theorem 1.1. We begin with results which follow by standard functional analysis from the definition of $H$, and then give more subtle estimates in terms of fractional, $G_{N^{-}}$invariant derivatives.

Assume that $G$ has stratified nilshadow and continue all notations of previous sections.

Lemma 3.1 The operator $H_{\varepsilon}$ generates a bounded holomorphic semigroup $S_{z}^{\varepsilon}=e^{-z H_{\varepsilon}}$ in $L_{2}$, for $z$ in a complex sector $\Lambda(\Omega)=\{z \in \mathbb{C}-\{0\}:|\arg z|<$ $\Omega\}$, where $\Omega \in\langle 0, \pi / 2]$ is independent of $\varepsilon>0$. Moreover $\left\|S_{z}^{\varepsilon}\right\|_{2 \rightarrow 2} \leq 1$ for all $z \in \Lambda(\Omega)$.

For each $j \in \mathbb{N}$ and $k \in\left\{1, \ldots, d^{\prime}\right\}$ one has estimates

$$
\left\|H_{\varepsilon}^{j} S_{t}^{\varepsilon}\right\|_{2 \rightarrow 2} \leq c_{j} t^{-j} \quad, \quad\left\|A_{k, \varepsilon} S_{t}^{\varepsilon}\right\|_{2 \rightarrow 2} \leq c t^{-1 / 2}
$$

for all $t>0, \varepsilon>0$. One has resolvent estimates

$$
\begin{aligned}
\left\|\left(\lambda I+H_{\varepsilon}\right)^{-1}\right\|_{2 \rightarrow 2} & \leq c \lambda^{-1} \\
\left\|A_{k, \varepsilon}\left(\lambda I+H_{\varepsilon}\right)^{-1}\right\|_{2 \rightarrow 2}+\left\|\left(\lambda I+H_{\varepsilon}\right)^{-1} A_{k, \varepsilon}\right\|_{2 \rightarrow 2} & \leq c \lambda^{-1 / 2} \\
\left\|A_{k, \varepsilon}\left(\lambda I+H_{\varepsilon}\right)^{-1} A_{l, \varepsilon}\right\|_{2 \rightarrow 2} & \leq c
\end{aligned}
$$

for all $\lambda>0, \varepsilon>0, k, l \in\left\{1, \ldots, d^{\prime}\right\}$.

Proof. First consider the case $\varepsilon=1$. That $H=H_{1}$ generates a contractive holomorphic semigroup in $L_{2}$ was given in Section 1. The estimates $\left\|H^{j} S_{t}\right\|_{2 \rightarrow 2} \leq c_{j} t^{-j},\left\|A_{k} S_{t}\right\|_{2 \rightarrow 2} \leq c t^{-1 / 2}$ and the resolvent estimates are straightforward consequences of the fact that $H$ generates a bounded holomorphic semigroup and/or the Gårding inequality (1.2).

For general $\varepsilon>0$, since $V_{\varepsilon}$ is a unitary transformation of $L_{2}$ it follows from (2.10) that $H_{\varepsilon}$ generates a holomorphic semigroup $S_{z}^{\varepsilon}, z \in \Lambda(\Omega)$. Moreover

$$
S_{z}^{\varepsilon}=V_{\varepsilon}^{-1} S_{\varepsilon^{-2} z} V_{\varepsilon} \quad, \quad\left(\lambda I+H_{\varepsilon}\right)^{-1}=\varepsilon^{2} V_{\varepsilon}^{-1}\left(\varepsilon^{2} \lambda I+H\right)^{-1} V_{\varepsilon},
$$

for all $z \in \Lambda(\Omega), \lambda>0$.

Once more applying (2.10) one easily obtains the estimates for $H_{\varepsilon}$ from the estimates for $H$, with constants $c$ independent of $\varepsilon$. 
Corollary 3.2 Given $P \in \mathcal{R}(G)$, there exists $\rho \geq 0$ such that

$$
\left\|P S_{t}\right\|_{2 \rightarrow 2} \leq c\left(1+t^{-\rho}\right) \quad \text { for all } t>0 \text {. }
$$

Proof. Since $P$ is a linear combination of $A^{\alpha}, \alpha \in J\left(d^{\prime}\right)$, from the bounds (1.3) we obtain the existence of $\rho \geq 0$ such that $\left\|P S_{t}\right\|_{2 \rightarrow 2} \leq c t^{-\rho}$ for all $0<t \leq 1$. For $t>1$ we have

$$
\left\|P S_{t}\right\|_{2 \rightarrow 2} \leq\left\|P S_{1}\right\|_{2 \rightarrow 2}\left\|S_{t-1}\right\|_{2 \rightarrow 2} \leq\left\|P S_{1}\right\|_{2 \rightarrow 2},
$$

and the corollary follows.

Let $r^{\prime}$ denote the rank of the generating list $a_{1}, \ldots, a_{d^{\prime}}$, that is, $r^{\prime}$ is the smallest positive integer $s$ such that $\mathfrak{g}$ is the linear span of all $a_{[\alpha]}$ with $\alpha \in J\left(d^{\prime}\right)$ and $1 \leq|\alpha| \leq s$. The following is the main result of this section.

Proposition 3.3 Given $\mu \in\left\langle 0,1 / r^{\prime}\right\rangle$, there exists $c=c(\mu)>0$ such that

$$
|q|^{-\mu}\left\|\left(I-L_{G_{N}}(q)\right) \chi\right\|_{2} \leq c\|\chi\|_{2}+c \sum_{k=1}^{d^{\prime}}\left\|A_{k, \varepsilon} \chi\right\|_{2}
$$

for all $\chi \in L_{2 ; \infty}, \varepsilon \in\langle 0,1]$ and $q \in Q_{N}$ with $q \neq e$.

Remark The value $1 / r^{\prime}$ in the proposition is sufficient for our purposes, but is not sharp. For example, let $\mathfrak{g}=\mathfrak{q}_{N}$ be a stratified Lie algebra with nilpotent rank $r \geq 2$, and choose the generating list $a_{k}=b_{k}, k \in\left\{1, \ldots, d_{1}\right\}$, of $\mathfrak{g}$. Then $r^{\prime}=r \geq 2$, but the estimate of the proposition holds with $\mu=1$, by (3.3) below.

Proof. The proof is based on a global derivative estimate for sublaplacians obtained in [1] (one can give another proof based only on local regularity estimates from [17], but then the details are more complicated).

We will need the following elementary inequality. Let $U$ be a unitary representation of $G$ in a Hilbert space $\mathcal{H}$, let $d U\left(a_{k}\right)$ denote the generator of the unitary group $t \in \mathbb{R} \mapsto U\left(\exp \left(-t a_{k}\right)\right), k \in\left\{1, \ldots, d^{\prime}\right\}$, and let $\mathcal{H}_{\infty} \subseteq \mathcal{H}$ denote the $C^{\infty}$ subspace consisting of $u \in \mathcal{H}$ for which the vector-valued mapping $g \mapsto U(g) u$ is smooth. Then

$$
\|(I-U(g)) u\|_{\mathcal{H}} \leq|g|_{a}\left(\sum_{k=1}^{d^{\prime}}\left\|d U\left(a_{k}\right) u\right\|_{\mathcal{H}}^{2}\right)^{1 / 2}
$$

for all $g \in G$ and $u \in \mathcal{H}_{\infty}$. The proof of (3.2) is straightforward from the definition of $|g|_{a}$ and the observation that if $\gamma:[0,1] \rightarrow G$ is a path from $e$ to $g^{-1}$ with $\dot{\gamma}(t)=\left.\sum_{k} \xi_{k}(t) A_{k}\right|_{\gamma(t)}$, then

$$
(I-U(g)) u=-\int_{0}^{1} d s \frac{d}{d s} U\left(\gamma(s)^{-1}\right) u=-\int_{0}^{1} d s \sum_{k=1}^{d^{\prime}} \xi_{k}(s) U\left(\gamma(s)^{-1}\right) d U\left(a_{k}\right) u \text {. }
$$


Let $\Delta=-\sum_{k=1}^{d^{\prime}} A_{k}^{2}$ be the sublaplacian on $G$ determined by $a_{1}, \ldots, a_{d^{\prime}}$, and $\Delta_{\varepsilon}=-\sum_{k=1}^{d^{\prime}} A_{k, \varepsilon}^{2}, \varepsilon>0$, the rescaled versions of $\Delta$. It is well known $[35,30]$ that the kernel $p_{t}$ of $e^{-t \Delta}$ satisfies Gaussian estimates of the form (1.4). Let $x \in \mathfrak{g}$ be arbitary and put $X=d L_{G}(x)$. From the Harnack inequalities of Theorem 7.7 of [1] we get an estimate

$$
\left|\left(X p_{t}\right)(g)\right| \leq c t^{-1 / 2} V_{a}(t)^{-1 / 2} e^{-b|g|_{a}^{2} / t}
$$

for all $t \geq 1$. Also, since $x$ is a linear combination of $a_{[\alpha]}$ with $1 \leq|\alpha| \leq r^{\prime}$, it follows from (1.3) that

$$
\left|\left(X p_{t}\right)(g)\right| \leq c^{\prime} t^{-r^{\prime} / 2} V_{a}(t)^{-1 / 2} e^{-b^{\prime}|g|_{a}^{2} / t}
$$

for $0<t<1$. By integration $\left\|X e^{-t \Delta}\right\|_{2 \rightarrow 2} \leq c\left(t^{-1 / 2}+t^{-r^{\prime} / 2}\right), t>0$, which by Lemma 2.12 yields

$$
\left\|B_{j}^{(N)} e^{-t \Delta}\right\|_{2 \rightarrow 2} \leq c\left(t^{-1 / 2}+t^{-r^{\prime} / 2}\right)
$$

for all $t>0$ and $j \in\left\{1, \ldots, d_{1}\right\}$. Using Lemma 2.15 we rescale to get

$$
\begin{aligned}
\left\|B_{j}^{(N)} e^{-t \Delta_{\varepsilon}}\right\|_{2 \rightarrow 2} & =\left\|\left(\varepsilon^{-1} V_{\varepsilon}^{-1} B_{j}^{(N)} V_{\varepsilon}\right)\left(V_{\varepsilon}^{-1} e^{-t \varepsilon^{-2} \Delta} V_{\varepsilon}\right)\right\|_{2 \rightarrow 2} \\
& =\varepsilon^{-1}\left\|B_{j}^{(N)} e^{-t \varepsilon^{-2} \Delta}\right\|_{2 \rightarrow 2} \\
& \leq c \varepsilon^{-1}\left(\left(t \varepsilon^{-2}\right)^{-1 / 2}+\left(t \varepsilon^{-2}\right)^{-r^{\prime} / 2}\right) \leq c\left(t^{-1 / 2}+t^{-r^{\prime} / 2}\right)
\end{aligned}
$$

for all $t>0, \varepsilon \in\langle 0,1]$ and $j \in\left\{1, \ldots, d_{1}\right\}$. Let us apply (3.2) to the representation $U(q)=L_{G_{N}}(q), q \in Q_{N}$, of $Q_{N}$ in $L_{2}$, with respect to the generating basis $b_{1}, \ldots, b_{d_{1}}$ of $\mathfrak{q}_{N}$. Since $q \mapsto|q|_{Q_{N}}=|q|$ is the associated modulus (Lemma 2.14) we obtain

$$
|q|^{-1}\left\|\left(I-L_{G_{N}}(q)\right) \chi\right\|_{2} \leq\left(\sum_{j=1}^{d_{1}}\left\|B_{j}^{(N)} \chi\right\|_{2}^{2}\right)^{1 / 2}
$$

for all $q \in Q_{N}, q \neq e$, and $\chi \in L_{2 ; \infty}$. Therefore

$$
|q|^{-1}\left\|\left(I-L_{G_{N}}(q)\right) e^{-t \Delta_{\varepsilon}}\right\|_{2 \rightarrow 2} \leq c\left(t^{-1 / 2}+t^{-r^{\prime} / 2}\right)
$$

for all $t>0, \varepsilon \in\langle 0,1]$ and $q \in Q_{N}, q \neq e$. Now fix $\mu \in\left\langle 0,1 / r^{\prime}\right\rangle$. Since

$$
\begin{aligned}
|q|^{-\mu}\left\|\left(I-L_{G_{N}}(q)\right) \chi\right\|_{2} & =\left(|q|^{-1}\left\|\left(I-L_{G_{N}}(q)\right) \chi\right\|_{2}\right)^{\mu}\left(\left\|\left(I-L_{G_{N}}(q)\right) \chi\right\|_{2}\right)^{1-\mu} \\
& \leq\left(|q|^{-1}\left\|\left(I-L_{G_{N}}(q)\right) \chi\right\|_{2}\right)^{\mu}\left(2\|\chi\|_{2}\right)^{1-\mu}
\end{aligned}
$$


and $e^{-t \Delta_{\varepsilon}}$ is a contraction semigroup on $L_{2}$, we deduce that

$$
|q|^{-\mu}\left\|\left(I-L_{G_{N}}(q)\right) e^{-t \Delta_{\varepsilon}}\right\|_{2 \rightarrow 2} \leq c\left(t^{-\mu / 2}+t^{-r^{\prime} \mu / 2}\right)
$$

for all $t>0$ and $\varepsilon \in\langle 0,1]$. The Laplace transform formula

$$
\left(I+\Delta_{\varepsilon}\right)^{-1 / 2}=c \int_{0}^{\infty} d t t^{-1 / 2} e^{-t} e^{-t \Delta_{\varepsilon}}
$$

then yields, because $r^{\prime} \mu<1$,

$$
|q|^{-\mu}\left\|\left(I-L_{G_{N}}(q)\right)\left(I+\Delta_{\varepsilon}\right)^{-1 / 2}\right\|_{2 \rightarrow 2} \leq c
$$

uniformly for all $\varepsilon \in\langle 0,1]$. Thus

$$
|q|^{-\mu}\left\|\left(I-L_{G_{N}}(q)\right) \chi\right\|_{2} \leq c\left\|\left(I+\Delta_{\varepsilon}\right)^{1 / 2} \chi\right\|_{2}
$$

for all $\varepsilon \in\langle 0,1], \chi \in L_{2 ; \infty}, q \in Q_{N}, q \neq e$. Since

$$
\left\|\left(I+\Delta_{\varepsilon}\right)^{1 / 2} \chi\right\|_{2}^{2}=\left(\left(I+\Delta_{\varepsilon}\right) \chi, \chi\right)=\|\chi\|_{2}^{2}+\sum_{k=1}^{d^{\prime}}\left\|A_{k, \varepsilon} \chi\right\|_{2}^{2},
$$

Proposition 3.3 follows.

We can combine Proposition 3.3 and Lemma 3.1 to get the following resolvent estimates. Introduce the operators

$$
L_{i}(t)=L_{G_{N}}\left(\exp _{G_{N}}\left(t b_{i}\right)\right) \quad, \quad L_{i}^{\prime}(t)=I-L_{i}(t)
$$

acting in $L_{2}$, for all $t \in \mathbb{R}$ and $i \in\{1, \ldots, d\}$. Note that $L_{i}(t)=e^{t B_{i}^{(N)}}$ is the one-parameter unitary group in $L_{2}$ generated by $-B_{i}^{(N)}$.

Corollary 3.4 There exist $c>0$ and $\mu \in\langle 0,1\rangle$ such that

$$
\begin{aligned}
& \left\|L_{i}^{\prime}(t)\left(I+H_{\varepsilon}\right)^{-1}\right\|_{2 \rightarrow 2}+\left\|L_{i}^{\prime}(t)\left(I+H_{\varepsilon}\right)^{-1} A_{k, \varepsilon}\right\|_{2 \rightarrow 2} \leq c|t|^{\mu} \\
& \left\|\left(I+H_{\varepsilon}\right)^{-1} L_{i}^{\prime}(t)\right\|_{2 \rightarrow 2}+\left\|A_{k, \varepsilon}\left(I+H_{\varepsilon}\right)^{-1} L_{i}^{\prime}(t)\right\|_{2 \rightarrow 2} \leq c|t|^{\mu}
\end{aligned}
$$

for all $i \in\{1, \ldots, d\}, k \in\left\{1, \ldots, d^{\prime}\right\}, t \in[-1,1]$ and $\varepsilon \in\langle 0,1]$.

Proof. The second estimate of the corollary follows by duality from the first estimate applied to the adjoint $H_{\varepsilon}^{*}$. To prove the first estimate, let $\varphi \in L_{2 ; \infty}$ and in Proposition 3.3 set $\chi=\left(I+H_{\varepsilon}\right)^{-1} \varphi$, or $\chi=\left(I+H_{\varepsilon}\right)^{-1} A_{k, \varepsilon} \varphi$, and $q=\exp _{G_{N}}\left(t b_{i}\right)$. Then the first estimate follows from Lemma 3.1, after noting that

$$
\left|\exp _{G_{N}}\left(t b_{i}\right)\right|=\left|\exp _{G_{N}}\left(|t| b_{i}\right)\right|=|t|^{1 / w(i)}\left|\exp _{G_{N}}\left(b_{i}\right)\right|
$$

for all $t \in \mathbb{R}$ by scaling (see Lemma 2.15(III)). 


\section{Proof of Theorem 1.1}

\subsection{The case of stratified nilshadow}

In this subsection we prove Theorem 1.1, assuming that $G$ has stratified nilshadow (Definition 2.6). The main effort of the proof is to establish the estimate of Proposition 4.1 below. This is a regularity estimate for $H_{\varepsilon}$, because it essentially states that a certain derivative of a function $\varphi$ is estimated in $L_{2}$ by derivatives, of "lower order", of $H_{\varepsilon} \varphi$ and $\varphi$. This interpretation of the proposition is made precise in (4.5) below. The proof of Theorem 1.1 is then completed using an inductive argument on the "order".

For the proposition we need spaces of fractional derivatives in the direction of $B_{i}^{(N)}, i \in\{1, \ldots, d\}$. Our spaces are special cases of the Lipschitz spaces for semigroups studied in [7], Chapter 3. Fix $i \in\{1, \ldots, d\}$ and for $\nu \in\langle 0,2\rangle$ define a seminorm $[\cdot]_{2 ; \nu, i}$ by

$$
[\varphi]_{2 ; \nu, i}=\left(\int_{0}^{1} d t t^{-1}\left(t^{-\nu}\left\|L_{i}^{\prime}(t)^{2} \varphi\right\|_{2}\right)^{2}\right)^{1 / 2} .
$$

The Lipschitz space is $L_{2 ; \nu, i}=\left\{\varphi \in L_{2}:[\varphi]_{2 ; \nu, i}<\infty\right\}$, with norm

$$
\|\varphi\|_{2 ; \nu, i}=\|\varphi\|_{2}+[\varphi]_{2 ; \nu, i}
$$

Then $L_{2 ; \nu, i}$ is a Banach space and when $\nu<1$ the norm $\|\cdot\|_{2 ; \nu, i}$ is equivalent to the norm

$$
\varphi \mapsto\|\varphi\|_{2}+\left(\int_{0}^{1} d t t^{-1}\left(t^{-\nu}\left\|L_{i}^{\prime}(t) \varphi\right\|_{2}\right)^{2}\right)^{1 / 2} .
$$

When $\nu=1$ the norm $\|\cdot\|_{2 ; 1, i}$ is equivalent to $\varphi \mapsto\|\varphi\|_{2}+\left\|B_{i}^{(N)} \varphi\right\|_{2}$. These equivalences follow by simple calculations from the spectral theorem for the skew-adjoint operator $B_{i}^{(N)}$ (compare, for example, Lemma 7.1 of [17]). It is also convenient to define $L_{2 ; 0, i}=L_{2}$ with norm $\|\cdot\|_{2,0, i}=\|\cdot\|_{2}$.

Finally, we need an ordering on the multi-indices in $J(d)$. If $\alpha=$ $\left(i_{1}, \ldots, i_{n}\right) \in J(d)$ define $[\alpha]_{j}$ as the number of $k \in\{1, \ldots, n\}$ such that $w\left(i_{k}\right)=j$ and set $[\alpha]=\left([\alpha]_{1}, \ldots,[\alpha]_{r}\right) \in \mathbb{N}_{0}^{r}$. Thus

$$
|\alpha|=\sum_{j=1}^{r}[\alpha]_{j} \quad \text { and } \quad\|\alpha\|=\sum_{j=1}^{r} j[\alpha]_{j} .
$$

Let $\prec$ be the dictionary order on the set $\{[\alpha]: \alpha \in J(d)\}$, so that $[\alpha] \prec[\beta]$ if and only if there exists an $l$ such that $[\alpha]_{l}<[\beta]_{l}$ and $[\alpha]_{j}=[\beta]_{j}$ for all $j<l$. Notice that $[\alpha]$ indicates the number of derivatives in $B^{(N) \alpha}$ in the direction of $\mathfrak{h}_{j}$ for each $j \in\{1, \ldots, r\}$. 
Proposition 4.1 Let $\mu \in\langle 0,1\rangle$ be as in Corollary 3.4. Suppose $\alpha \in J(\mathfrak{n})$, $i \in\left\{d_{0}+1, \ldots, d\right\}, \nu \in\langle 0,1]$, and $\nu^{\prime} \in[0, \nu\rangle$ such that $\nu-\nu^{\prime} \leq \mu$. If $\nu=1$ let $\delta \in\langle 0,1\rangle$ be arbitrary, or if $\nu<1$ set $\delta=0$. Then there exists $c=c\left(\alpha, i, \nu, \nu^{\prime}, \delta\right)>0$ such that

$$
\begin{aligned}
\| B^{(N) \alpha} & \varphi\left\|_{2 ; \nu, i}+\sum_{k}\right\| B^{(N) \alpha} A_{k, \varepsilon} \varphi \|_{2 ; \nu, i} \leq \\
\leq & c\left\|B^{(N) \alpha} H_{\varepsilon} \varphi\right\|_{2 ; \nu^{\prime}, i}+c\left\|B^{(N) \alpha} \varphi\right\|_{2 ; \nu^{\prime}, i}+c\left\|B^{(N) \alpha} \varphi\right\|_{2} \\
& +c \sum_{l}\left\|B^{(N) \alpha} A_{l, \varepsilon} \varphi\right\|_{2}+c \sum_{\beta}\left(\left\|B^{(N) \beta} \varphi\right\|_{2 ; \nu, i}+\sum_{l}\left\|B^{(N) \beta} A_{l, \varepsilon} \varphi\right\|_{2 ; \nu, i}\right) \\
& +c \sum_{j}\left(\left\|B_{j}^{(N)} B^{(N) \alpha} \varphi\right\|_{2 ; \delta, i}+\sum_{l}\left\|B_{j}^{(N)} B^{(N) \alpha} A_{l, \varepsilon} \varphi\right\|_{2 ; \delta, i}\right)
\end{aligned}
$$

for all $\varepsilon \in\langle 0,1]$ and $\varphi \in L_{2 ; \infty}$, with the sums over $k, l \in\left\{1, \ldots, d^{\prime}\right\}, \beta \in$ $J(\mathfrak{n})$ with $|\beta|=|\alpha|,[\beta] \prec[\alpha]$, and $j \in\left\{d_{0}+1, \ldots, d\right\}$ with $w(j)>w(i)$.

The proof is based on the following formal identity. For an operator $P$, a function $\varphi$ and

$$
H_{\varepsilon}=-\sum_{k, l} c_{k l} A_{k, \varepsilon} A_{l, \varepsilon}
$$

we have

$$
\begin{aligned}
P \varphi= & \left(I+H_{\varepsilon}\right)^{-1} P\left(I+H_{\varepsilon}\right) \varphi+\left(I+H_{\varepsilon}\right)^{-1}\left[H_{\varepsilon}, P\right] \varphi \\
= & \left(I+H_{\varepsilon}\right)^{-1} P\left(I+H_{\varepsilon}\right) \varphi-\sum_{k, l} c_{k l}\left(I+H_{\varepsilon}\right)^{-1}\left[A_{k, \varepsilon}, P\right] A_{l, \varepsilon} \varphi \\
& \quad-\sum_{k, l} c_{k l}\left(I+H_{\varepsilon}\right)^{-1} A_{k, \varepsilon}\left[A_{l, \varepsilon}, P\right] \varphi .
\end{aligned}
$$

To calculate $\left[A_{k, \varepsilon}, P\right]$ we require two lemmas on commutator identities, which exploit the nilpotency of $\mathfrak{q}_{N}$. Let $\operatorname{ad}_{N}$ denote the adjoint representation of $\mathfrak{g}_{N}$. The basic observation is that

$$
\left(\operatorname{ad}_{N} b_{i}\right)\left(\mathfrak{g}_{N}\right) \subseteq \mathfrak{q}_{N ; w(i)+1} \subseteq \mathfrak{n}
$$

for all $i=1, \ldots, d$. Indeed, the first inclusion follows because $b_{i} \in \mathfrak{q}_{N ; w(i)}$, $\left[\mathfrak{q}_{N ; w(i)}, \mathfrak{q}_{N}\right]_{N} \subseteq \mathfrak{q}_{N ; w(i)+1}$ and $\left[\mathfrak{q}_{N ; w(i)}, \mathfrak{m}\right]_{N} \subseteq\left[\mathfrak{q}_{N}, \mathfrak{m}\right]_{N}=\{0\}$. The second inclusion follows because $w(i) \geq 1$ so that $\mathfrak{q}_{N ; w(i)+1} \subseteq \mathfrak{q}_{N ; 2} \subseteq \mathfrak{n}$. 
Lemma 4.2 If $X^{(N)}=d L_{G_{N}}(x), x \in \mathfrak{g}_{N}$, and $\alpha \in J(d)$, then there are real constants $c_{\beta}$ such that

$$
\left[X^{(N)}, B^{(N) \alpha}\right]=\sum_{\beta} c_{\beta} B^{(N) \beta},
$$

with the sum over $\beta \in J(d)$ satisfying $|\beta|=|\alpha|,\|\beta\| \geq\|\alpha\|+1$ and $[\beta] \prec[\alpha]$. Moreover, if $\alpha \in J(\mathfrak{n})$ then $\beta \in J(\mathfrak{n})$ in the sum.

Proof. If $\alpha=\left(i_{1}, \ldots, i_{n}\right)$ then

$$
\left[X^{(N)}, B^{(N) \alpha}\right]=\sum_{q=1}^{n} B_{i_{1}}^{(N)} \ldots B_{i_{q-1}}^{(N)}\left[X^{(N)}, B_{i_{q}}^{(N)}\right] B_{i_{q+1}}^{(N)} \ldots B_{i_{n}}^{(N)} .
$$

From the observation preceding the lemma, there are $c_{q, j} \in \mathbb{R}$ with

$$
\left[x, b_{i_{q}}\right]_{N}=\sum_{j} c_{q, j} b_{j}
$$

with the sum over $j \in\left\{d_{0}+1, \ldots, d\right\}$ such that $w(j)>w\left(i_{q}\right)$. Hence

$$
\left[X^{(N)}, B_{i_{q}}^{(N)}\right]=\sum_{j} c_{q, j} B_{j}^{(N)}
$$

and the lemma follows.

Lemma 4.3 If $X^{(N)}=d L_{G_{N}}(x), x \in \mathfrak{g}_{N}$, and $i \in\{1, \ldots, d\}$, then there exist real constants $c_{q, j}$ such that

$$
\left[X^{(N)}, L_{i}(t)\right]=L_{i}(t) \sum_{q=1}^{r-1} \sum_{j} c_{q, j} t^{q} B_{j}^{(N)}
$$

for all $t \in \mathbb{R}$, with the sum over $j \in\left\{d_{0}+1, \ldots, d\right\}$ satisfying $w(j)>w(i)$.

Proof. One has

$$
\left[X^{(N)}, L_{i}(t)\right]=L_{i}(t)\left(L_{i}(-t) X^{(N)} L_{i}(t)-X^{(N)}\right)
$$

and

$$
L_{i}(-t) X^{(N)} L_{i}(t)=d L_{G_{N}}\left(\operatorname{Ad}_{N}\left(\exp _{G_{N}}\left(-t b_{i}\right)\right) x\right)=d L_{G_{N}}\left(e^{-t \operatorname{ad}_{N} b_{i}} x\right)
$$

where $\operatorname{Ad}_{N}$ denotes the adjoint representation of $G_{N}$ in $\mathfrak{g}_{N}$. Since $\mathfrak{q}_{N}$ has nilpotent rank $r$ and from the observations preceding Lemma 4.2, we have

$$
e^{-t \operatorname{ad}_{N} b_{i}} x=x+\sum_{q=1}^{r-1}(q !)^{-1}(-1)^{q} t^{q}\left(\operatorname{ad}_{N} b_{i}\right)^{q} x=x+\sum_{q=1}^{r-1} \sum_{j} c_{q, j} t^{q} b_{j}
$$

with the sum over $j \in\left\{d_{0}+1, \ldots, d\right\}$ satisfying $w(j)>w(i)$. The lemma follows. 
Proof of Proposition 4.1. Let us fix $\mu, \alpha, i, \nu, \nu^{\prime}, \delta$ as in the hypothesis. By $\sum_{j}$ we will understand a sum over all $j \in\left\{d_{0}+1, \ldots, d\right\}$ satisfying $w(j)>w(i)$, and by $\sum_{\beta}$ a sum over all $\beta \in J(\mathfrak{n})$ with $|\beta|=|\alpha|$ and $[\beta] \prec[\alpha]$.

For $t \in \mathbb{R}$ we write $L(t)=L_{i}(t), L^{\prime}(t)=L_{i}^{\prime}(t)$, and consider the operator $D_{t}=L^{\prime}(t)^{2} B^{(N) \alpha}$. We put $P=D_{t}$ in the identity (4.1) and apply Lemma 3.1 and Corollary 3.4 to obtain

$$
\begin{aligned}
\left\|D_{t} \varphi\right\|_{2} \leq & \left\|\left(I+H_{\varepsilon}\right)^{-1} L^{\prime}(t)\right\|_{2 \rightarrow 2}\left\|L^{\prime}(t) B^{(N) \alpha}\left(I+H_{\varepsilon}\right) \varphi\right\|_{2} \\
& +c \sum_{k, l}\left\|\left(I+H_{\varepsilon}\right)^{-1}\right\|_{2 \rightarrow 2}\left\|\left[A_{k, \varepsilon}, D_{t}\right] A_{l, \varepsilon} \varphi\right\|_{2} \\
& +c \sum_{k, l}\left\|\left(I+H_{\varepsilon}\right)^{-1} A_{k, \varepsilon}\right\|_{2 \rightarrow 2}\left\|\left[A_{l, \varepsilon}, D_{t}\right] \varphi\right\|_{2} \\
\leq & c t^{\mu}\left\|L^{\prime}(t) B^{(N) \alpha}\left(I+H_{\varepsilon}\right) \varphi\right\|_{2} \\
& +c \sum_{k, l}\left\|\left[A_{k, \varepsilon}, D_{t}\right] A_{l, \varepsilon} \varphi\right\|_{2}+c \sum_{k}\left\|\left[A_{k, \varepsilon}, D_{t}\right] \varphi\right\|_{2}
\end{aligned}
$$

for all $t \in[0,1], \varphi \in L_{2 ; \infty}$ and $\varepsilon \in\langle 0,1]$. Next we derive an estimate for the commutator $\left[A_{k, \varepsilon}, D_{t}\right]$. From $(2.11)$ we get

$$
\left[A_{k, \varepsilon}, D_{t}\right]=\sum_{u=-p}^{d} \varepsilon^{-1+w(u)}\left[\sigma_{k, u}^{(\varepsilon)} B_{u}^{(N)}, D_{t}\right]=\sum_{u=1}^{d} \varepsilon^{-1+w(u)} \sigma_{k, u}^{(\varepsilon)}\left[B_{u}^{(N)}, D_{t}\right]
$$

because (2.12) implies that $D_{t}$ commutes with multiplication by the functions $\sigma_{k, u}^{(\varepsilon)}$, and because $[\mathfrak{m}, \mathfrak{n}]_{N}=\{0\}$ implies that $\left[B_{u}^{(N)}, D_{t}\right]=0$ when $u \in\{-p, \ldots, 0\}$. We have

$$
\left[B_{u}^{(N)}, D_{t}\right]=L^{\prime}(t)^{2}\left[B_{u}^{(N)}, B^{(N) \alpha}\right]+\left[B_{u}^{(N)}, L^{\prime}(t)^{2}\right] B^{(N) \alpha}
$$

where the term $\left[B_{u}^{(N)}, B^{(N) \alpha}\right]$ may be expanded using Lemma 4.2. Also

$$
\begin{aligned}
{\left[B_{u}^{(N)}, L^{\prime}(t)^{2}\right] } & =2 L^{\prime}(t)\left[B_{u}^{(N)}, L^{\prime}(t)\right]+\left[\left[B_{u}^{(N)}, L^{\prime}(t)\right], L^{\prime}(t)\right] \\
& =2 L^{\prime}(t)\left[B_{u}^{(N)}, L(t)\right]+\left[\left[B_{u}^{(N)}, L(t)\right], L(t)\right],
\end{aligned}
$$

and applications of Lemma 4.3 yield that

$$
\left\|\left[B_{u}^{(N)}, L^{\prime}(t)^{2}\right] \chi\right\|_{2} \leq c t \sum_{j}\left\|L^{\prime}(t) B_{j}^{(N)} \chi\right\|_{2}+c t^{2} \sum_{j}\left\|B_{j}^{(N)} \chi\right\|_{2}
$$

for all $t \in[0,1]$ and $\chi \in L_{2 ; \infty}$. 
Combining these observations gives the estimate

$$
\begin{aligned}
\left\|\left[A_{k, \varepsilon}, D_{t}\right] \chi\right\|_{2} \leq & c^{\prime} \sum_{u=1}^{d}\left\|\left[B_{u}^{(N)}, D_{t}\right] \chi\right\|_{2} \\
\leq & c \sum_{\beta}\left\|L^{\prime}(t)^{2} B^{(N) \beta} \chi\right\|_{2} \\
& +c t \sum_{j}\left\|L^{\prime}(t) B_{j}^{(N)} B^{(N) \alpha} \chi\right\|_{2}+c t^{2} \sum_{j}\left\|B_{j}^{(N)} B^{(N) \alpha} \chi\right\|_{2}
\end{aligned}
$$

for all $t \in[0,1], \chi \in L_{2 ; \infty}, k \in\left\{1, \ldots, d^{\prime}\right\}$, and $\varepsilon \in\langle 0,1]$.

Substitute (4.4) with $\chi=\varphi$ and $\chi=A_{l, \varepsilon} \varphi$ into (4.3), and observe that $t^{-\nu} t^{\mu} \leq t^{-\nu^{\prime}}$ to get

$$
\begin{aligned}
& t^{-\nu}\left\|D_{t} \varphi\right\|_{2} \leq c t^{-\nu^{\prime}}\left\|L^{\prime}(t) B^{(N) \alpha}\left(I+H_{\varepsilon}\right) \varphi\right\|_{2} \\
& +c t^{-\nu} \sum_{\beta}\left(\left\|L^{\prime}(t)^{2} B^{(N) \beta} \varphi\right\|_{2}+\sum_{l}\left\|L^{\prime}(t)^{2} B^{(N) \beta} A_{l, \varepsilon} \varphi\right\|_{2}\right) \\
& +c t^{1-\nu} \sum_{j}\left(\left\|L^{\prime}(t) B_{j}^{(N)} B^{(N) \alpha} \varphi\right\|_{2}+\sum_{l}\left\|L^{\prime}(t) B_{j}^{(N)} B^{(N) \alpha} A_{l, \varepsilon} \varphi\right\|_{2}\right) \\
& +c t^{2-\nu} \sum_{j}\left(\left\|B_{j}^{(N)} B^{(N) \alpha} \varphi\right\|_{2}+\sum_{l}\left\|B_{j}^{(N)} B^{(N) \alpha} A_{l, \varepsilon} \varphi\right\|_{2}\right)
\end{aligned}
$$

for all $t \in\langle 0,1]$ and $\varepsilon \in\langle 0,1]$. Take norms in $L_{2}\left(\langle 0,1\rangle ; t^{-1} d t\right)$ on both sides of this inequality. Note that if $\nu<1$ then $t \mapsto t^{1-\nu}$ is in $L_{2}\left(\langle 0,1\rangle ; t^{-1} d t\right)$, while if $\nu=1$ then $t^{1-\nu} \leq t^{-\delta}$. In either case one concludes that

$$
\begin{aligned}
{\left[B^{(N) \alpha} \varphi\right]_{2 ; \nu, i} \leq } & c\left\|B^{(N) \alpha}\left(I+H_{\varepsilon}\right) \varphi\right\|_{2 ; \nu^{\prime}, i} \\
& +c \sum_{\beta}\left(\left\|B^{(N) \beta} \varphi\right\|_{2 ; \nu, i}+\sum_{l}\left\|B^{(N) \beta} A_{l, \varepsilon} \varphi\right\|_{2 ; \nu, i}\right) \\
& +c \sum_{j}\left(\left\|B_{j}^{(N)} B^{(N) \alpha} \varphi\right\|_{2 ; \delta, i}+\sum_{l}\left\|B_{j}^{(N)} B^{(N) \alpha} A_{l, \varepsilon} \varphi\right\|_{2 ; \delta, i}\right)
\end{aligned}
$$

for all $\varepsilon \in\langle 0,1]$ and $\varphi \in L_{2 ; \infty}$. Therefore

$$
\left\|B^{(N) \alpha} \varphi\right\|_{2 ; \nu, i} \leq(*)
$$

where $(*)$ denotes the right side in the estimate of Proposition 4.1. 
To complete the proof of the proposition it suffices to show that

$$
\left[B^{(N) \alpha} A_{h, \varepsilon} \varphi\right]_{2 ; \nu, i} \leq(*)
$$

for all $h \in\left\{1, \ldots, d^{\prime}\right\}$ and $\varepsilon \in\langle 0,1]$. This is achieved by a small variation of the above arguments. Note that

$$
\left\|D_{t} A_{h, \varepsilon} \varphi\right\|_{2} \leq\left\|A_{h, \varepsilon} D_{t} \varphi\right\|_{2}+\left\|\left[A_{h, \varepsilon}, D_{t}\right] \varphi\right\|_{2}
$$

where the second term on the right side is estimated by (4.4). To bound the first term we premultiply (4.1) by $A_{h, \varepsilon}$ and get

$$
\begin{aligned}
\left\|A_{h, \varepsilon} D_{t} \varphi\right\|_{2} \leq & \left\|A_{h, \varepsilon}\left(I+H_{\varepsilon}\right)^{-1} L^{\prime}(t)\right\|_{2 \rightarrow 2}\left\|L^{\prime}(t) B^{(N) \alpha}\left(I+H_{\varepsilon}\right) \varphi\right\|_{2} \\
& +c \sum_{k, l}\left\|A_{h, \varepsilon}\left(I+H_{\varepsilon}\right)^{-1}\right\|_{2 \rightarrow 2}\left\|\left[A_{k, \varepsilon}, D_{t}\right] A_{l, \varepsilon} \varphi\right\|_{2} \\
& +c \sum_{k, l}\left\|A_{h, \varepsilon}\left(I+H_{\varepsilon}\right)^{-1} A_{k, \varepsilon}\right\|_{2 \rightarrow 2}\left\|\left[A_{l, \varepsilon}, D_{t}\right] \varphi\right\|_{2} .
\end{aligned}
$$

In this inequality all terms on the right side can be estimated thanks to Lemma 3.1, Corollary 3.4 and (4.4). Reasoning as before, we get an estimate for $t^{-\nu}\left\|D_{t} A_{h, \varepsilon} \varphi\right\|_{2}, t \in\langle 0,1]$, which yields $\left[B^{(N) \alpha} A_{h, \varepsilon} \varphi\right]_{2 ; \nu, i} \leq(*)$ after taking norms in $L_{2}\left(\langle 0,1\rangle ; t^{-1} d t\right)$. This finishes the proof of Proposition 4.1.

We now complete the proof of Theorem 1.1 in the case that $G$ has stratified nilshadow. Let $\mu \in\langle 0,1\rangle$ be as in Corollary 3.4. Choose $q \in \mathbb{N}$ with $q \mu>1$ and fix $\mu_{0}, \mu_{1}, \ldots, \mu_{q}$ satisfying

$$
0=\mu_{0}<\mu_{1}<\ldots<\mu_{q}=1
$$

and $\mu_{j}-\mu_{j-1}<\mu$ for $j \in\{1, \ldots, q\}$. Let $\mathcal{J}$ denote the set of all triples $\mathcal{A}=(\alpha, \nu, i)$ where $\alpha \in J(\mathfrak{n}), \nu \in\left\{0, \mu_{1}, \ldots, \mu_{q-1}\right\}$ and $i \in\left\{d_{0}+1, \ldots, d\right\}$. We write $|\mathcal{A}|=|\alpha|$ and set

$$
|\varphi|_{\mathcal{A}}=\left\|B^{(N) \alpha} \varphi\right\|_{2 ; \nu, i}
$$

for all $\varphi \in L_{2 ; \infty}$. Define an $r$-tuple $[\mathcal{A}]=\left(\rho_{1}, \ldots, \rho_{r}\right)$, the "order" of $\mathcal{A}$, by

$$
[\mathcal{A}]=[\alpha]+\nu \mathbf{e}_{w(i)} \in \mathbb{R}^{r}
$$

where $\mathbf{e}_{j}$ denotes the $j$-th standard basis vector in $\mathbb{R}^{r}$. Then $\mathcal{O}=\{[\mathcal{A}]: \mathcal{A} \in \mathcal{J}\}$ is a countable subset of $\mathbb{R}^{r}$. Moreover $\mathcal{O}$ is a well-ordered set under the dictionary order $\prec$, where $\left(\rho_{1}, \ldots, \rho_{r}\right) \prec\left(\rho_{1}^{\prime}, \ldots, \rho_{r}^{\prime}\right)$ means that there is an $l$ with $\rho_{l}<\rho_{l}^{\prime}$ and $\rho_{j}=\rho_{j}^{\prime}$ for all $j<l$. 
With this notation, Proposition 4.1 yields the following inequality. For each $\mathcal{A} \in \mathcal{J}$ with $[\mathcal{A}] \neq 0$, there exists $c=c(\mathcal{A})>0$ such that

$$
|\varphi|_{\mathcal{A}}+\sum_{k}\left|A_{k, \varepsilon} \varphi\right|_{\mathcal{A}} \leq c \sum_{\substack{[\mathcal{B}]<[\mathcal{A}] \\|\mathcal{B}| \leq|\mathcal{A}|+1}}\left(\left|H_{\varepsilon} \varphi\right|_{\mathcal{B}}+|\varphi|_{\mathcal{B}}+\sum_{k}\left|A_{k, \varepsilon} \varphi\right|_{\mathcal{B}}\right)
$$

for all $\varphi \in L_{2 ; \infty}$ and $\varepsilon \in\langle 0,1]$. Note that the sum on the right side is over a finite number of $\mathcal{B} \in \mathcal{J}$, and sums over $k$ are understood to be over $k \in\left\{1, \ldots, d^{\prime}\right\}$. Remark that in case $\mathcal{A}=(\alpha, 0, i)$, we derive (4.5) from the $\nu=1$ case of Proposition 4.1 and the fact that for each $j \in\left\{d_{0}+1, \ldots, d\right\}$ the norm $\|\cdot\|_{2 ; 1, j}$ is equivalent to $\varphi \mapsto\|\varphi\|_{2}+\left\|B_{j}^{(N)} \varphi\right\|_{2}$.

Next we claim that for each $\mathcal{A} \in \mathcal{J}$, there exists $c=c(\mathcal{A})>0$ and $j=j(\mathcal{A}) \in \mathbb{N}$ such that

$$
|\varphi|_{\mathcal{A}}+\sum_{k}\left|A_{k, \varepsilon} \varphi\right|_{\mathcal{A}} \leq c\left(\|\varphi\|_{2}+\sum_{l=1}^{j}\left\|H_{\varepsilon}^{l} \varphi\right\|_{2}\right)
$$

for all $\varphi \in L_{2 ; \infty}$ and $\varepsilon \in\langle 0,1]$. Indeed, when $[\mathcal{A}]=0$ then $|\cdot|_{\mathcal{A}}=\|\cdot\|_{2}$, and the claim (with $j=1$ ) is a consequence of the resolvent estimates of Lemma 3.1. Suppose $[\mathcal{A}] \neq 0$. By induction on the well-ordered set $\mathcal{O}$, we may assume the claim is true for all $\mathcal{B} \in \mathcal{J}$ with $[\mathcal{B}] \prec[\mathcal{A}]$. But applying (4.5) and then the induction hypothesis, we see that the claim is true for $\mathcal{A}$. This proves (4.6) for all $\mathcal{A}$.

In (4.6), choose $\mathcal{A}$ of the form $\mathcal{A}=(\alpha, 0, i)$ and set $\varphi=S_{1}^{\varepsilon} \psi, \psi \in L_{2}$. (Note that $S_{t}$ maps $L_{2}$ into $L_{2 ; \infty}$ for $t>0$ as a consequence of local estimates (1.3), and that $V_{\varepsilon}\left(L_{2 ; \infty}\right) \subseteq L_{2 ; \infty}$ by Lemma 2.15 (I). Hence $\varphi \in L_{2 ; \infty}$ by scaling.) From Lemma 3.1 we have for each $l \in \mathbb{N}$ an estimate $\left\|H^{l} S_{1}^{\varepsilon} \psi\right\|_{2} \leq$ $c_{l}\|\psi\|_{2}$. We conclude that for each $\alpha \in J(\mathfrak{n})$, there is $c(\alpha)>0$ such that

$$
\left\|B^{(N) \alpha} S_{1}^{\varepsilon}\right\|_{2 \rightarrow 2}+\sum_{k}\left\|B^{(N) \alpha} A_{k, \varepsilon} S_{1}^{\varepsilon}\right\|_{2 \rightarrow 2} \leq c(\alpha)
$$

for all $\varepsilon \in\langle 0,1]$. Now rescale using Lemma 2.15 and the identities (2.10), (3.1) to get

$$
\left\|B^{(N) \alpha} S_{t}\right\|_{2 \rightarrow 2}+t^{1 / 2} \sum_{k}\left\|B^{(N) \alpha} A_{k} S_{t}\right\|_{2 \rightarrow 2} \leq c(\alpha) t^{-\|\alpha\| / 2}
$$

for all $t \geq 1$. Let $y_{1}, \ldots, y_{m}$ be as in the statement of Theorem 1.1. Then $y_{i} \in \mathfrak{n} \cap \mathfrak{q}_{N ; j_{i}}$ and it follows by Lemma 2.12 that

$$
Y_{i}=\sum_{l} \sigma_{i, l} B_{l}^{(N)}
$$

where $\sigma_{i, l} \in \mathcal{E}$ and the sum is over $l \in\left\{d_{0}+1, \ldots, d\right\}$ with $w(l) \geq j_{i}$. 
By Corollary 2.13, the $B_{l}^{(N)}$ for $l>d_{0}$ commute with multiplication by elements of $\mathcal{E}$. Thus we may express

$$
Y_{1} \ldots Y_{m}=\sum_{\alpha} \sigma_{\alpha} B^{(N) \alpha}
$$

where $\sigma_{\alpha} \in \mathcal{E}$ and the sum is over $\alpha \in J(\mathfrak{n})$ with $|\alpha|=m$ and $\|\alpha\| \geq$ $w=j_{1}+\cdots+j_{m}$. Therefore the bounds of Theorem 1.1 follow immediately from (4.7).

\section{Remarks}

(a) We have assumed that $\varphi \in L_{2 ; \infty}$ in Proposition 4.1, but the proof shows that the estimate of the proposition is valid whenever $\varphi \in L_{2}$ and the right side of the estimate is finite. Then (4.5) holds whenever $\varphi \in L_{2}$ and the right side of (4.5) is finite, and we deduce that (4.6) holds for all $\varphi \in L_{2}$ in the domain of $H_{\varepsilon}^{j}$. Thus we see that the local regularity result $S_{t}\left(L_{2}\right) \subseteq L_{2 ; \infty}(t>0)$ is not essential for the proof of (4.7).

(b) Let us justify Remark (d) in Section 1. Suppose that $G$ is stratified nilpotent, with $\left\{\mathfrak{h}_{j}\right\}$ a stratification of the nilpotent Lie algebra $\mathfrak{g}=\mathfrak{q}_{N}=\mathfrak{n}$, and that $a_{1}, \ldots, a_{d^{\prime}}$ is a basis of the "first slice" $\mathfrak{h}_{1}$ (thus $d^{\prime}=d_{1}$, and we may assume that $a_{k}=b_{k}$ for $\left.k \in\left\{1, \ldots, d^{\prime}\right\}\right)$. Then $G=G_{\varepsilon}=G_{N}=Q_{N}$ as Lie groups, $A_{k, \varepsilon}=A_{k}, H_{\varepsilon}=H$, for all $\varepsilon>0$, and $B^{(N) \alpha}=B^{\alpha}$ for all $\alpha \in J(d)=J(\mathfrak{n})$. Proposition 3.3 holds with $\mu=1$ : indeed, this is just the elementary estimate (3.3). The estimate (4.6), Remark (a) above, and interpolation between powers of $H$, yield that

$$
\left\|B^{\alpha} \varphi\right\|_{2} \leq c\left(\|\varphi\|_{2}+\left\|H^{j} \varphi\right\|_{2}\right)
$$

for all $\varphi \in L_{2}$ in the domain of $H^{j}$. This estimate is a special case of results of [27] for homogeneous hypoelliptic operators, but is here derived independently. The estimates (4.9), together with the fact that $H$ is homogeneous of order 2, are sufficient to apply Sobolev-embedding, scaling and perturbation arguments, as in [3], Section 2, or [14],Section 2. The conclusion, as in these references, is that $S_{t}$ has a kernel $K_{t}$ which, together with all its $B^{\alpha}$-derivatives, satisfies global Gaussian bounds.

\subsection{The general case}

We now prove Theorem 1.1 for general $G$ without an assumption of stratified nilshadow. Our method is to realize $G$ as a quotient of a group $\widetilde{G}$ with stratified nilshadow, and transfer the estimates of the theorem from $\widetilde{G}$ to $G$ with a standard transference result (see [9]). The details are as follows. 
If $\mathfrak{g}$ is the Lie algebra of $G$, Proposition 10.7 gives the existence of a type $R$ Lie algebra $\tilde{\mathfrak{g}}$, with stratified nilshadow in the sense of Definition 2.6, and a surjective homomorphism $\pi: \tilde{\mathfrak{g}} \rightarrow \mathfrak{g}$. Let $\widetilde{G}$ be the connected, simply connected group of polynomial growth with Lie algebra $\tilde{\mathfrak{g}}$. Since $\widetilde{G}$ is simply connected, there is a surjective homomorphism $\Lambda: \widetilde{G} \rightarrow G$ such that $d \Lambda=\pi$.

As usual we write $A_{k}=d L_{G}\left(a_{k}\right), k \in\left\{1, \ldots, d^{\prime}\right\}$ and consider

$$
H=-\sum_{k, l=1}^{d^{\prime}} c_{k l} A_{k} A_{l}
$$

acting in $L_{2}(G ; d g)$, with $K_{t}$ the convolution kernel of $S_{t}=e^{-t H}$. Choose elements $\tilde{a}_{k} \in \tilde{\mathfrak{g}}$ with $\pi\left(\tilde{a}_{k}\right)=a_{k}, k \in\left\{1, \ldots, d^{\prime}\right\}$. Let $\tilde{a}_{d^{\prime}+1}, \ldots, \tilde{a}_{d^{\prime \prime}}$ be a vector space basis for the kernel $\mathfrak{e}=\pi^{-1}\{0\}$ of $\pi$, where $d^{\prime \prime}=d^{\prime}+\operatorname{dim}(\mathfrak{e})$. If $\mathfrak{h}$ denotes the Lie subalgebra of $\tilde{\mathfrak{g}}$ generated by $\tilde{a}_{1}, \ldots, \tilde{a}_{d^{\prime \prime}}$, then $\pi(\mathfrak{h})=\mathfrak{g}$ and hence

$$
\tilde{\mathfrak{g}}=\pi^{-1}(\mathfrak{g})=\pi^{-1}(\pi(\mathfrak{h}))=\mathfrak{h}+\mathfrak{e}=\mathfrak{h} \quad .
$$

Therefore $\tilde{a}_{1}, \ldots, \tilde{a}_{d^{\prime \prime}}$ generate $\tilde{\mathfrak{g}}$. Write $\widetilde{A}_{k}=d L_{\widetilde{G}}\left(\tilde{a}_{k}\right)$ and consider the subelliptic operator

$$
\widetilde{H}=-\sum_{k, l=1}^{d^{\prime}} c_{k l} \widetilde{A}_{k} \widetilde{A}_{l}-\sum_{k=d^{\prime}+1}^{d^{\prime \prime}} \widetilde{A}_{k}^{2}
$$

acting in $L_{2}(\widetilde{G} ; d \tilde{g})$, where $d \tilde{g}$ is Haar measure for $\widetilde{G}$. Let $\widetilde{K}_{t}$ denote the convolution kernel of the semigroup $\widetilde{S}_{t}=e^{-t \widetilde{H}}, t>0$. Since $\widetilde{G}$ has stratified nilshadow, the estimates of Theorem 1.1 are valid for $\widetilde{S}_{t}$.

For $\varphi \in C^{\infty}(G)$ one has

$$
\widetilde{A}_{k}(\varphi \circ \Lambda)=\left(A_{k} \varphi\right) \circ \Lambda
$$

for $k \in\left\{1, \ldots, d^{\prime}\right\}$, and $\widetilde{A}_{k}(\varphi \circ \Lambda)=0$ for $k \in\left\{d^{\prime}+1, \ldots, d^{\prime \prime}\right\}$. Hence

$$
\widetilde{H}(\varphi \circ \Lambda)=(H \varphi) \circ \Lambda
$$

for $\varphi \in C^{\infty}(G)$. For $1 \leq q<\infty$, let $U$ be the isometric representation of $\widetilde{G}$ in $L_{q}(G)$ given by $U(\tilde{g})=L_{G}(\Lambda \tilde{g}), \tilde{g} \in \widetilde{G}$. For $\psi \in L_{1}(\widetilde{G})$ define the operator $U(\psi)$ acting in $L_{q}(G)$ by

$$
U(\psi)=\int_{\widetilde{G}} d \tilde{g} \psi(\widetilde{g}) U(\widetilde{g})
$$

It follows from (4.10) and standard group-theoretic considerations, as in [22], that

$$
S_{t}=U\left(\widetilde{K}_{t}\right)
$$


as operators in $L_{q}(G)$, and more generally

$$
X_{1} \cdots X_{m} S_{t}=U\left(\widetilde{X}_{1} \cdots \widetilde{X}_{m} \widetilde{K}_{t}\right)
$$

where $X_{j}=d L_{G}\left(\pi\left(\tilde{x}_{j}\right)\right)=d U\left(\tilde{x}_{j}\right)$ and $\widetilde{X}_{j}=d L_{\widetilde{G}}\left(\tilde{x}_{j}\right)$ for arbitrary elements $\tilde{x}_{1}, \ldots, \tilde{x}_{m}$ of $\tilde{\mathfrak{g}}$. Note that $\widetilde{X}_{1} \cdots \widetilde{X}_{m} \widetilde{K}_{t}$ is the convolution kernel of the operator $\widetilde{X}_{1} \cdots \widetilde{X}_{m} \widetilde{S}_{t}$, and is an element of $L_{1}(\widetilde{G})$ (as follows from the Gaussian estimates (1.3) applied to $\left.\widetilde{K}_{t}\right)$. Therefore we can apply a transference theorem, Theorem 2.4 of [9], to get

$$
\left\|X_{1} \cdots X_{m} S_{t}\right\|_{q \rightarrow q} \leq\left\|\widetilde{X}_{1} \cdots \widetilde{X}_{m} \widetilde{S}_{t}\right\|_{q \rightarrow q}
$$

(with the operator norm in $L_{q}(G)$ on the left side, and in $L_{q}(\widetilde{G})$ on the right side). From Proposition 10.7 we have $\pi\left(\tilde{\mathfrak{q}}_{N ; j}\right)=\mathfrak{q}_{N ; j}$ for all $j \in \mathbb{N}$, and $\pi^{-1}(\mathfrak{n})=\tilde{\mathfrak{n}}$. Therefore if $x_{1}, \ldots, x_{m} \in \mathfrak{n}$ with $x_{i} \in \mathfrak{q}_{N ; j_{i}}$, then there exist $\tilde{x}_{i} \in \tilde{\mathfrak{n}} \cap \tilde{\mathfrak{q}}_{N ; j_{i}}$ such that $x_{i}=\pi\left(\tilde{x}_{i}\right)$ for all $i \in\{1, \ldots, m\}$. Then applying Theorem 1.1 to $\widetilde{H}$, we deduce from (4.11) that

$$
\left\|X_{1} \cdots X_{m} S_{t}\right\|_{2 \rightarrow 2} \leq c t^{-w / 2}
$$

for $t \geq 1$, with $w=j_{1}+\cdots+j_{m}$. Similarly, we can get the second estimate of Theorem 1.1 by putting $X_{m}=A_{k}, \widetilde{X}_{m}=\widetilde{A}_{k}$ in (4.11). This ends the proof of Theorem 1.1.

\section{Proof of Theorem 1.2}

Let $G_{\mathfrak{s}}$ be the analytic subgroup of $G$ with Lie algebra $\mathfrak{s}$. The key to proving Theorem 1.2 is the remark that $G_{\mathfrak{s}}$ is compact and normal, because $\mathfrak{s}$ is a type $R$ semisimple ideal of $\mathfrak{g}$.

To avoid triviality, we assume that $\operatorname{dim}(\mathfrak{s}) \geq 1$. Let $Y=d L_{G}(y)$ where $y \in \mathfrak{s}$, and let $P, Q \in \mathcal{R}(G)$. Our first goal is to obtain the $L_{2}$-estimate

$$
\left\|P Y Q S_{t}\right\|_{2 \rightarrow 2} \leq c e^{-\sigma t}
$$

for some $c, \sigma>0$ and all $t \geq 1$.

Let $\mathcal{P}: L_{2} \rightarrow L_{2}$ denote the orthogonal projection onto the subspace of $L_{2}$ consisting of functions which are constant along the cosets $g G_{\mathfrak{s}}=G_{\mathfrak{s}} g$ for all $g \in G$. If $\varphi \in L_{2}$ is continuous and compactly supported, it is easy to see that

$$
(\mathcal{P} \varphi)(g)=\int_{G_{\mathfrak{s}}} d s \varphi(g s)=\int_{G_{\mathfrak{s}}} d s \varphi(s g)
$$

for all $g \in G$, where $d s$ is Haar measure for $G_{\mathfrak{s}}$ normalized so that $d s\left(G_{\mathfrak{s}}\right)=1$. The main step to prove (5.1) is the following lemma, which extends arguments of [23, Section 4]. 
Lemma 5.1 The projection $\mathcal{P}: L_{2} \rightarrow L_{2}$ commutes with $X=d L_{G}(x)$ for any $x \in \mathfrak{g}$, and hence commutes with $H$ and $S_{t}$. There exist $c, \omega>0$ such that

$$
\left\|S_{t}(I-\mathcal{P})\right\|_{2 \rightarrow 2} \leq c e^{-\omega t}
$$

for all $t>0$.

Proof. The first formula for $\mathcal{P} \varphi$ easily implies that $L_{G}(g) \mathcal{P}=\mathcal{P} L_{G}(g)$ for all $g \in G$. Therefore $\mathcal{P}$ commutes with $d L_{G}(x)$, with $H$ and with $S_{t}=e^{-t H}$.

Let $e_{1}, \ldots, e_{q}$ be a vector space basis for $\mathfrak{s}$ and set $E_{j}=d L_{G}\left(e_{j}\right), F_{j}=$ $d L_{G_{\mathfrak{s}}}\left(e_{j}\right)$. Since $G_{\mathfrak{s}}$ is compact and connected, the operator

$$
H_{\mathfrak{s}}=-\sum_{j=1}^{q} F_{j}^{2}
$$

acting in $L_{2}\left(G_{\mathfrak{s}} ; d s\right)$ has a compact resolvent. Moreover, there exists $\lambda>0$ such that $H_{\mathfrak{s}} \geq \lambda I$ on the orthogonal complement in $L_{2}\left(G_{\mathfrak{s}}\right)$ of the constant functions (see [30], Section IV.3).

Let $\varphi \in(I-\mathcal{P})\left(C_{c}^{\infty}(G)\right) \subseteq C_{c}^{\infty}(G)$ and define $\varphi_{g} \in C^{\infty}\left(G_{\mathfrak{s}}\right)$ by $\varphi_{g}(s)=$ $\varphi(s g)$ for all $g \in G, s \in G_{\mathfrak{s}}$. Observe that $F_{j}\left(\varphi_{g}\right)=\left(E_{j} \varphi\right)_{g}$, and that $\varphi_{g}$ is orthogonal to constants because $0=(\mathcal{P} \varphi)(g)=\int_{G_{\mathfrak{s}}} d s \varphi_{g}(s)$. For $\psi \in C_{c}(G)$ we have the integration formula

$$
\int_{G} d g \psi(g)=\int_{G / G_{\mathfrak{s}}} d \dot{g} \int_{G_{\mathfrak{s}}} d s \psi(s g)
$$

where $\dot{g}=g G_{\mathfrak{s}}=G_{\mathfrak{s}} g$ and $d \dot{g}$ is Haar measure for the group $G / G_{\mathfrak{s}}$ (see for example [24]). Combining these remarks we obtain

$$
\begin{aligned}
\sum_{j=1}^{q}\left\|E_{j} \varphi\right\|_{2}^{2} & =\sum_{j=1}^{q} \int_{G / G_{\mathfrak{s}}} d \dot{g} \int_{G_{\mathfrak{s}}} d s\left|\left(E_{j} \varphi\right)(s g)\right|^{2} \\
& =\sum_{j=1}^{q} \int_{G / G_{\mathfrak{s}}} d \dot{g} \int_{G_{\mathfrak{s}}} d s\left|\left(F_{j} \varphi_{g}\right)(s)\right|^{2} \\
& \geq \lambda \int_{G / G_{\mathfrak{s}}} d \dot{g} \int_{G_{\mathfrak{s}}} d s\left|\left(\varphi_{g}\right)(s)\right|^{2}=\lambda\|\varphi\|_{2}^{2}
\end{aligned}
$$

for all $\varphi \in(I-\mathcal{P}) C_{c}^{\infty}$. Next, the argument of Lemma 4.2 of [23] shows that for each $\alpha \in J\left(d^{\prime}\right)$ with $|\alpha| \geq 1$, there is $l=l(\alpha) \in \mathbb{N}$ such that

$$
\left\|A^{\alpha} \varphi\right\|_{2} \leq \varepsilon\|\varphi\|_{2}+c_{\varepsilon}\left\|H^{l} \varphi\right\|_{2}
$$

for all $\varphi \in C_{c}^{\infty}$ and $\varepsilon>0$, where $c_{\varepsilon}>0$ depends on $\varepsilon$. 
Since each $E_{j}$ is expressible as a linear combination of $A^{\alpha}$ with $|\alpha| \geq 1$, it easily follows that there is an $l \in \mathbb{N}$ such that

$$
\sum_{j=1}^{q}\left\|E_{j} \varphi\right\|_{2}^{2} \leq \varepsilon\|\varphi\|_{2}^{2}+c_{\varepsilon}\left\|H^{l} \varphi\right\|_{2}^{2}
$$

for all $\varphi \in C_{c}^{\infty}$ and $\varepsilon>0$, with $c_{\varepsilon}>0$ depending on $\varepsilon$. Fixing $\varepsilon<\lambda$ we obtain the existence of $\lambda^{\prime}>0$ such that

$$
\left\|H^{l} \varphi\right\|_{2} \geq \lambda^{\prime}\|\varphi\|_{2}
$$

for all $\varphi \in(I-\mathcal{P}) C_{c}^{\infty}$. Note that $S_{t}^{\prime}=S_{t}(I-\mathcal{P})$ is the holomorphic semigroup generated by the operator $H(I-\mathcal{P})$ in $L_{2}$. Then the estimate $\left\|S_{t}^{\prime}\right\|_{2 \rightarrow 2} \leq c e^{-\omega t}$ is a consequence of (5.2) and a spectral lemma for holomorphic semigroups, Lemma 4.2 of [14].

Remark The lemma and its proof remain valid with $G_{\mathfrak{s}}$ replaced by any compact, connected normal subgroup $K$ of $G$, with $\mathcal{P}$ the projection corresponding to $K$.

Continuing with the proof of (5.1), since $y \in \mathfrak{s}$ we have $Y \mathcal{P}=0$. Hence

$$
P Y Q S_{t} \mathcal{P}=P(Y \mathcal{P}) Q S_{t}=0
$$

because $\mathcal{P}$ commutes with $Q$ and with $S_{t}$. From Corollary 3.2 we have for some $\rho>0$ an estimate $\left\|P Y Q S_{t}\right\|_{2 \rightarrow 2} \leq c\left(1+t^{-\rho}\right)$ for all $t>0$. Then if $\delta \in\langle 0,1\rangle$ we see that

$$
\begin{aligned}
\left\|P Y Q S_{t}\right\|_{2 \rightarrow 2} & \leq\left\|P Y Q S_{\delta t}\right\|_{2 \rightarrow 2}\left\|S_{(1-\delta) t}(I-\mathcal{P})\right\|_{2 \rightarrow 2} \\
& \leq c^{\prime}\left(1+(\delta t)^{-\rho}\right) e^{-\omega(1-\delta) t}
\end{aligned}
$$

for all $t>0$, where $\omega$ is as in Lemma 5.1. After fixing $\delta$ this implies (5.1) with $\sigma=(1-\delta) \omega$ (in particular, we see that $\sigma$ can be chosen arbitrarily close to but less than $\omega$ ).

Finally, we obtain the kernel bounds of Theorem 1.2 by interpolating (5.1) with the local bounds (1.3). Write $P^{\prime}=P Y Q \in \mathcal{R}(G)$. Integration of (1.3) yields for some $\omega>0$ that

$$
\left\|K_{t}\right\|_{2} \leq c t^{-D_{a} / 4} e^{\omega t}
$$

for all $t>0$. Let $\delta \in\langle 0,1 / 4\rangle$ to be chosen. By convolution and applying (5.1) we get

$$
\begin{aligned}
\left\|P^{\prime} K_{t}\right\|_{\infty} & \leq\left\|P^{\prime} K_{(1-\delta) t}\right\|_{2}\left\|K_{\delta t}\right\|_{2} \\
& \leq\left\|P^{\prime} S_{(1-2 \delta) t}\right\|_{2 \rightarrow 2}\left\|K_{\delta t}\right\|_{2}^{2} \leq c e^{-\sigma_{1} t}
\end{aligned}
$$

for some $\sigma_{1}>0$ and all $t \geq 2$, provided $\delta$ is chosen sufficiently small. 
From (1.3) we get the existence of $\rho, \omega, b>0$ such that

$$
\left|\left(P^{\prime} K_{t}\right)(g)\right| \leq c t^{-\rho} e^{\omega t} e^{-b|g|_{a}^{2} / t}
$$

for all $t>0$ and $g \in G$. Let $\delta \in\langle 0,1\rangle$ to be chosen, and observe that

$$
\begin{aligned}
\left|\left(P^{\prime} K_{t}\right)(g)\right| & \leq\left(\left\|P^{\prime} K_{t}\right\|_{\infty}\right)^{1-\delta}\left|\left(P^{\prime} K_{t}\right)(g)\right|^{\delta} \\
& \leq c e^{-(1-\delta) \sigma_{1} t}(\delta t)^{-\rho} e^{\delta \omega t} e^{-\delta b|g|_{a}^{2} / t}
\end{aligned}
$$

for all $t \geq 2$ and $g \in G$. Fixing $\delta$ small enough so that

$$
\sigma_{2}:=(1-\delta) \sigma_{1}-\delta \omega>0,
$$

we finish the proof of Theorem 1.2.

\section{Proof of Theorem 1.4}

It is not difficult to obtain the correct decrease in $t$ for the $L_{\infty}$ norm of the kernels in Theorem 1.4. Indeed, for any right-invariant differential operator $P$, one can use the convolution identity $P K_{t}=P K_{t / 2} * K_{t / 2}$ to estimate

$$
\left\|P K_{t}\right\|_{\infty} \leq\left\|P K_{t / 2}\right\|_{2}\left\|K_{t / 2}\right\|_{2} \leq\left\|P S_{t / 4}\right\|_{2 \rightarrow 2}\left\|K_{t / 4}\right\|_{2}\left\|K_{t / 2}\right\|_{2}
$$

Thus, when $K_{t}$ satisfies Gaussian bounds (1.4), and $y_{1}, \ldots, y_{m}$ are as in the statement of the theorem, one can deduce that $\left\|Y_{1} \ldots Y_{m} K_{t}\right\|_{\infty} \leq c t^{-w / 2} t^{-D / 2}$ for $t \geq 1$. But to derive Gaussian bounds, we will need more elaborate arguments.

First, the following lemma gives the case $m=0$ of Theorem 1.4. Since the proof follows the method of Saloff-Coste explained in $[31,35,30]$, we do not repeat the details.

Lemma 6.1 Suppose that the global Gaussian bounds (1.4) are satisfied. Then there exist $c, b>0$ such that

$$
\left|\left(A_{k} K_{t}\right)(g)\right| \leq c t^{-1 / 2} V_{a}(t)^{-1 / 2} e^{-b|g|_{a}^{2} / t}
$$

for all $k \in\left\{1, \ldots, d^{\prime}\right\}, t>0$ and $g \in G$.

Note that in the statement of Theorem 1.4, the modulus $|\cdot|_{a}$ can equivalently be replaced by the modulus $|\cdot|$, because $t \geq 1$ and the moduli are equivalent at infinity (Lemma 2.14(I)). In this rest of this section we prefer to work with $|\cdot|$.

For $r>0$ let $B(r)=\left\{g \in G_{N}:|g|<r\right\}$. The next lemma, which is a slight elaboration of results of [23], shows that the modulus can be approximated by "cutoff" functions whose $G_{N}$-derivatives satisfy nice estimates. 
Lemma 6.2 There exist $\tau_{1}>\tau_{2}>0$ and a family $\left\{\eta_{R}\right\}_{R \geq 1}$ of $C^{\infty}$ functions on $G_{N}$ satisfying $0 \leq \eta_{R} \leq 1$, the support of $\eta_{R}$ is contained in $B\left(\tau_{1} R\right)$, and $\eta_{R}(g)=1$ if $g \in B\left(\tau_{2} R\right)$, for all $R \geq 1$. For each $\alpha \in J(-p, d)$ there exists $c_{\alpha} \geq 0$ such that

$$
\left\|B^{(N) \alpha} \eta_{R}\right\|_{\infty} \leq c_{\alpha} R^{-\|\alpha\|}
$$

for all $R \geq 1$, with $c_{\alpha}=0$ if $\alpha \notin J(d)$.

Proof. Consider the subgroup $Q_{N}$ of $G_{N}$, the derivatives $\widetilde{B}_{j}=d L_{Q_{N}}\left(b_{j}\right)$, $j \in\left\{1, \ldots, d_{1}\right\}$, and the corresponding modulus $q \mapsto|q|_{Q_{N}}$ defined in Subsection 2.4. Let $B^{\prime}(t)=\left\{q \in Q_{N}:|q|_{Q_{N}}<t\right\}$ for $t>0$. Since $Q_{N}$ is nilpotent, by results of [23] there exist $\tau_{1}^{\prime}>\tau_{2}^{\prime}>0$ and a family $\left\{\eta_{R}^{\prime}\right\}_{R \geq 1}$ of $C^{\infty}$ functions on $Q_{N}$ with $0 \leq \eta_{R}^{\prime} \leq 1$, the support of $\eta_{R}^{\prime}$ is contained in $B^{\prime}\left(\tau_{1}^{\prime} R\right)$, and $\eta_{R}^{\prime}(g)=1$ for $g \in B^{\prime}\left(\tau_{2}^{\prime} R\right)$. For each $\alpha \in J\left(d_{1}\right)$ there is $c_{\alpha}^{\prime}>0$ with

$$
\left\|\widetilde{B}^{\alpha} \eta_{R}^{\prime}\right\|_{\infty} \leq c_{\alpha}^{\prime} R^{-|\alpha|} \text { for } R \geq 1 .
$$

Define $\eta_{R} \in C^{\infty}\left(G_{N}\right)$ by setting $\eta_{R}\left(m *_{N} q\right)=\eta_{R}^{\prime}(q)$ for $m \in M, q \in Q_{N}$. It easily follows from the compactness of $M$ and Lemma 2.14 that $\eta_{R}$ have the desired support properties, for some $\tau_{1}>\tau_{2}>0$. Since the $\eta_{R}$ are constant in the direction of $M$, one has $B_{i}^{(N)} \eta_{R}=0$ for $i \in\{-p, \ldots, 0\}$. But $B_{i}^{(N)}$ commutes with $B_{j}^{(N)}$ when $i \leq 0$ and $j \geq 1$, so that $B^{(N) \alpha} \eta_{R}=0$ whenever $\alpha \in J(-p, d)-J(d)$.

The proof of Proposition 2.3 shows that for each $j \in \mathbb{N}, \mathfrak{q}_{N ; j}$ is spanned by all commutators $\left[b_{i_{1}},\left[\cdots,\left[b_{i_{s-1}}, b_{i_{s}}\right]_{N} \cdots\right]_{N}\right]_{N}$ with $s \geq j$ and $i_{1}, \ldots, i_{s} \in$ $\left\{1, \ldots, d_{1}\right\}$. It easily follows that for each $\beta \in J(d)$ one can write

$$
B^{(N) \beta}=\sum_{\alpha} r_{\alpha} B^{(N) \alpha}
$$

with $r_{\alpha} \in \mathbb{R}$ and the sum over a finite number of $\alpha \in J\left(d_{1}\right)$ with $|\alpha| \geq$ $\|\beta\|$. But (6.1) implies that $\left\|B^{(N) \alpha} \eta_{R}\right\|_{\infty} \leq c_{\alpha}^{\prime} R^{-|\alpha|}$ for $R \geq 1, \alpha \in J\left(d_{1}\right)$. Therefore $\left\|B^{(N) \beta} \eta_{R}\right\|_{\infty} \leq c_{\beta} R^{-\|\beta\|}$.

Let us note a consequence of Lemma 6.2 for $G$-invariant derivatives. If $x \in \mathfrak{g}, X=d L_{G}(x)$ and $j \in \mathbb{N}$ such that $x \in \mathfrak{m} \oplus \mathfrak{q}_{N ; j}$, then by Lemma 2.12 we can express

$$
X=\sum_{i=-p}^{0} r_{i} B_{i}^{(N)}+\sum_{l} \sigma_{l} B_{l}^{(N)}
$$

where $r_{i} \in \mathbb{R}, \sigma_{l} \in \mathcal{E}$ and the sum is over $l \in\{1, \ldots, d\}$ with $w(l) \geq j$. Then Lemma 6.2 yields an estimate

$$
\left\|X \eta_{R}\right\|_{\infty} \leq c R^{-j}
$$

for all $R \geq 1$. 
Define $\psi_{R}=R\left(1-\eta_{R}\right)$ and let $U_{\rho}$ denote the operator of multiplication by $e^{\rho \psi_{R}}$, for any $R \geq 1$ and $\rho \geq R^{-1}$. Unless otherwise stated, subsequent estimates involving $U_{\rho}$ are understood to hold for all $R \geq 1$ and $\rho>0$ such that $\rho \geq R^{-1}$, though for brevity we have omitted $R$ from the notation. Next, we show that Gaussian estimates are equivalent to $U_{\rho}$-weighted $L_{2}$ estimates.

Lemma 6.3 Let $\delta \geq 0$ and let $P \in \mathcal{R}(G)$ be a right-invariant differential operator on $G$. The following conditions are equivalent:

(I) There exist $c, b>0$ such that

$$
\left|K_{t}(g)\right|+t^{\delta}\left|\left(P K_{t}\right)(g)\right| \leq c t^{-D / 2} e^{-b|g|^{2} / t}
$$

for all $t \geq 1$ and $g \in G$;

(II) There exists $c, \omega>0$ such that

$$
\left\|U_{\rho} K_{t}\right\|_{2}+t^{\delta}\left\|U_{\rho} P K_{t}\right\|_{2} \leq c t^{-D / 4} e^{\omega \rho^{2} t}
$$

for all $t \geq 1, \rho>0, R \geq 1$ with $\rho \geq R^{-1}$.

Proof. " (I) $\Rightarrow$ (II)" With $\tau_{2}>0$ as in Lemma 6.2 we have

$$
\psi_{R}(g) \leq \tau_{2}^{-1}|g|
$$

for all $g \in G, R \geq 1$, by considering the two cases $|g|<\tau_{2} R,|g| \geq \tau_{2} R$. Let $b$ be as in Condition (I), fix $b^{\prime} \in\langle 0, b\rangle$ and choose an $\omega>0$ large enough so that

$$
-\left(b-b^{\prime}\right) s^{2} / t \leq \omega \rho^{2} t-\tau_{2}^{-1} \rho s
$$

for all $s \geq 0, t>0, \rho>0$. Setting $s=|g|$, we obtain from (I) that

$$
\left|\left(U_{\rho} K_{t}\right)(g)\right| \leq c t^{-D / 2} e^{-b|g|^{2} / t} e^{\tau_{2}^{-1} \rho|g|} \leq c t^{-D / 2} e^{-b^{\prime}|g|^{2} / t} e^{\omega \rho^{2} t}
$$

for all $t \geq 1$ and $\rho>0$. Integration of these bounds yields $\left\|U_{\rho} K_{t}\right\|_{2} \leq$ $c^{\prime} t^{-D / 4} e^{\overline{\omega \rho}^{2} t}$ for $t \geq 1, \rho>0$. Similarly we obtain the desired estimate for $\left\|U_{\rho} P K_{t}\right\|_{2}$.

"(II) $\Rightarrow(\mathrm{I})$ ". From the convolution identity $P K t=P K_{t / 2} * K_{t / 2}$ and (II) we have

$$
\begin{aligned}
\left\|P K_{t}\right\|_{\infty} & \leq\left\|P K_{t / 2}\right\|_{2}\left\|K_{t / 2}\right\|_{2} \\
& \leq\left\|U_{\rho} P K_{t / 2}\right\|_{2}\left\|U_{\rho} K_{t / 2}\right\|_{2} \leq c t^{-\delta} t^{-D / 2} e^{\omega \rho^{2} t}
\end{aligned}
$$

for all $t \geq 2$ and $\rho \in\langle 0,1\rangle$. Letting $\rho \rightarrow 0$ gives

$$
\left\|P K_{t}\right\|_{\infty} \leq c t^{-\delta} t^{-D / 2}
$$

for $t \geq 2$. 
Next, let $\tau_{1}$ be as in Lemma 6.2, let $g \in G$ with $|g| \geq 3 \tau_{1}$ and set $R=\left(3 \tau_{1}\right)^{-1}|g|$. Then $|g| / 2>\tau_{1} R$. For any $h \in G$ we have $|g| \leq|h|+\left|h^{-1} g\right|$; considering the two cases $|h| \geq|g| / 2,\left|h^{-1} g\right| \geq|g| / 2$, we deduce from the properties of $\eta_{R}$ that

$$
|g|=3 \tau_{1} R=3 \tau_{1} \max \left\{\psi_{R}(h), \psi_{R}\left(h^{-1} g\right)\right\} \leq 3 \tau_{1}\left(\psi_{R}(h)+\psi_{R}\left(h^{-1} g\right)\right)
$$

for all $h \in G$. Therefore

$$
\begin{aligned}
e^{\rho|g|}\left|\left(P K_{t}\right)(g)\right| & \leq \int d h e^{3 \tau_{1} \rho \psi_{R}(h)}\left|\left(P K_{t / 2}\right)(h)\right| e^{3 \tau_{1} \rho \psi_{R}\left(h^{-1} g\right)} \mid\left(K_{t / 2}\left(h^{-1} g\right) \mid\right. \\
& \leq\left\|U_{3 \tau_{1} \rho} P K_{t / 2}\right\|_{2}\left\|U_{3 \tau_{1} \rho} K_{t / 2}\right\|_{2} \\
& \leq c t^{-\delta} t^{-D / 2} e^{\omega \rho^{2} t}
\end{aligned}
$$

for all $t \geq 2,|g| \geq 3 \tau_{1}$, and $\rho>0$ satisfying $3 \tau_{1} \rho \geq R^{-1}=3 \tau_{1}|g|^{-1}$. Fixing $\sigma>0$ sufficiently small and setting $\rho=\sigma|g| / t$, we obtain an estimate

$$
\left|\left(P K_{t}\right)(g)\right| \leq c t^{-\delta} t^{-D / 2} e^{-b|g|^{2} / t}
$$

for all $t \geq 2$ and $g \in G$ such that $\sigma|g|^{2} / t \geq 1$. If $t \geq 2$ and $\sigma|g|^{2} / t \leq 1$ then an estimate of the same form follows from (6.3). Repeating the above reasoning with $P=I, \delta=0$, we complete the proof of (I).

To prove Theorem 1.4, suppose that $K_{t}$ satisfies global Gaussian bounds (1.4) and let $m \in \mathbb{N}, y_{1}, \ldots, y_{m} \in \mathfrak{n}^{\prime}, j_{1}, \ldots, j_{m}, w=j_{1}+\cdots+j_{m}$ be as in the statement of the theorem. We prove the desired Gaussian estimate for the kernel $Y_{1} \cdots Y_{m} A_{k} K_{t}$; the similar estimate for $Y_{1} \cdots Y_{m} K_{t}$ is left to the reader. Note that Lemma 6.3 (with $P=0$ ) yields an estimate

$$
\left\|U_{\rho} K_{t}\right\|_{2} \leq c t^{-D / 4} e^{\omega \rho^{2} t}
$$

for all $t \geq 1, \rho \geq R^{-1}$.

For $X=d L_{G}(x), x \in \mathfrak{g}$, and a smooth function $\varphi$ on $G$, we define $X_{\rho} \varphi=U_{\rho} X U_{\rho}^{-1} \varphi ;$ then

$$
X_{\rho} \varphi=X \varphi-\rho\left(X \psi_{R}\right) \varphi .
$$

Write $P=Y_{2} \cdots Y_{m} A_{k} \in \mathcal{R}(G)$, let $t>0, R \geq 1, \rho \geq R^{-1}$, and observe that

$$
\begin{aligned}
\| U_{\rho} Y_{1} Y_{2} \cdots & Y_{m} A_{k} K_{t} \|_{2}^{2}= \\
& =\left(Y_{1} P K_{t}, U_{2 \rho} Y_{1} P K_{t}\right) \\
& =\left(Y_{1} P K_{t},\left(Y_{1}\right)_{2 \rho} U_{2 \rho} P K_{t}\right) \\
& =-\left(Y_{1}^{2} P K_{t}, U_{2 \rho} P K_{t}\right)-2 \rho\left(Y_{1} P K_{t},\left(Y_{1} \psi_{R}\right) U_{2 \rho} P K_{t}\right) \\
& \leq\left\|Y_{1}^{2} P K_{t}\right\|_{2}\left\|U_{2 \rho} P K_{t}\right\|_{2}+2 \rho\left\|Y_{1} \psi_{R}\right\|_{\infty}\left\|Y_{1} P K_{t}\right\|_{2}\left\|U_{2 \rho} P K_{t}\right\|_{2} .
\end{aligned}
$$


By induction on $m$, and Lemma 6.1, we may assume that the correct Gaussian estimate holds for $P K_{t}$. Thus Lemma 6.3 gives for some $\omega^{\prime}>0$ an estimate

$$
\left\|U_{2 \rho} P K_{t}\right\|_{2} \leq c t^{-1 / 2} t^{-\left(w-j_{1}\right) / 2} t^{-D / 4} e^{\omega^{\prime} \rho^{2} t}
$$

for $t \geq 1, \rho \geq R^{-1}$. Next, application of Corollary 1.3 and the assumption that $K_{t}$ satisfies global Gaussian bounds yields

$$
\begin{aligned}
\left\|Y_{1} P K_{t}\right\|_{2} & \leq\left\|Y_{1} P S_{t / 2}\right\|_{2 \rightarrow 2}\left\|K_{t / 2}\right\|_{2} \leq c t^{-1 / 2} t^{-w / 2} t^{-D / 4} \\
\left\|Y_{1}^{2} P K_{t}\right\|_{2} & \leq\left\|Y_{1}^{2} P S_{t / 2}\right\|_{2 \rightarrow 2}\left\|K_{t / 2}\right\|_{2} \leq c t^{-1 / 2} t^{-\left(w+j_{1}\right) / 2} t^{-D / 4}
\end{aligned}
$$

for all $t \geq 1$. Since $y_{1} \in \mathfrak{s} \oplus \mathfrak{q}_{N ; j_{1}} \subseteq \mathfrak{m} \oplus \mathfrak{q}_{N ; j_{1}}$, we obtain from (6.2) a bound

$$
\left\|Y_{1} \psi_{R}\right\|_{\infty} \leq c R^{-j_{1}+1} \leq c \rho^{j_{1}-1}
$$

for $\rho \geq R^{-1}$. Inserting the above estimates in (6.4) gives for some $\omega^{\prime \prime}>0$ that

$$
\left\|U_{\rho} Y_{1} \cdots Y_{m} A_{k} K_{t}\right\|_{2} \leq c t^{-1 / 2} t^{-w / 2} t^{-D / 4} e^{\omega^{\prime \prime} \rho^{2} t}
$$

for all $t \geq 1$. Applying Lemma 6.3, we finish the proof of Theorem 1.4.

\section{Proof of Theorem 1.5}

Let us prove the theorem for the operators $Y_{1} \cdots Y_{m} H^{-w / 2, \infty}$; the arguments for the operators $H^{-w / 2, \infty} Y_{1} \cdots Y_{m}$ are quite similar.

The difficulty with applying standard singular integration theory to $Y_{1} \cdots Y_{m} H^{-w / 2, \infty}$ is that its two-variable integral kernel may not satisfy standard Hölder continuity estimates with respect to the first variable (for a related discussion, see [1, Section 1]). We will avoid this problem by treating the operators $B^{(N) \alpha} H^{-\|\alpha\| / 2, \infty}, \alpha \in J(\mathfrak{n})$, whose kernels turn out to satisfy Lipschitz estimates with respect to either variable.

Assume that $K_{t}$ satisfies Gaussian bounds (1.4). Of course the kernel $K_{t}^{*}(g)=\overline{K_{t}\left(g^{-1}\right)}$ of the adjoint $H^{*}=-\sum_{k, l} \overline{c_{l k}} A_{k} A_{l}$ then satisfies the same bounds, so that Theorem 1.4 can be applied to both $H$ and $H^{*}$.

Proposition 7.1 For each $\alpha \in J(\mathfrak{n})$ with $|\alpha| \geq 1$, the transform $R_{\alpha}=$ $B^{(N) \alpha} H^{-\|\alpha\| / 2, \infty}$ is bounded in $L_{q}, 1<q<\infty$, and from $L_{1}$ to weak- $L_{1}$.

Proof. Fix $\alpha \in J(\mathfrak{n})$ with $|\alpha| \geq 1$. Let $K_{t}^{\prime}$ denote the two-variable integral kernel of $S_{t}$, so that $K_{t}^{\prime}(g ; h)=K_{t}\left(g h^{-1}\right)$ for $g, h \in G$. Let $d_{a}$ be the standard right-invariant distance on $G$ given by $d_{a}(g ; h)=\left|g h^{-1}\right|_{a}$ for $g, h \in G$. Consider the kernel $B^{(N) \alpha} K_{t}^{\prime}(g ; h)=B^{(N) \alpha(g)} K_{t}^{\prime}(g ; h)$, where we use superscript $(g)$ or $(h)$ to denote differentiation with respect to the variables $g, h$ respectively. 
We first prove estimates

$$
\begin{gathered}
\left|B^{(N) \alpha} K_{t}^{\prime}(g ; h)\right| \leq c t^{-\|\alpha\| / 2} t^{-D / 2} e^{-b d_{a}(g ; h)^{2} / t} \\
\left|B^{(N) \alpha} K_{t}^{\prime}(g ; h)-B^{(N) \alpha} K_{t}^{\prime}(g ; l)\right| \leq c d_{a}(h ; l) t^{-(\|\alpha\|+1) / 2} t^{-D / 2} e^{-b d_{a}(g ; h)^{2} / t} \\
\left|B^{(N) \alpha} K_{t}^{\prime}(h ; g)-B^{(N) \alpha} K_{t}^{\prime}(l ; g)\right| \leq c d_{a}(h ; l) t^{-(\|\alpha\|+1) / 2} t^{-D / 2} e^{-b d_{a}(g ; h)^{2} / t}
\end{gathered}
$$

for all $t \geq 1$ and $g, h, l \in G$ such that $d_{a}(h ; l) \leq(1 / 2) d_{a}(g ; h)+t^{1 / 2}$. Indeed, using the results of Subsection 2.4 one obtains for each $\gamma \in J(\mathfrak{n})$ an expression

$$
B^{(N) \gamma}=\sum_{\delta} \sigma_{\gamma, \delta} B^{\delta}
$$

where $\sigma_{\gamma, \delta} \in \mathcal{E}$ and with summation over $\delta \in J(\mathfrak{n})$ satisfying $|\delta|=|\gamma|$ and $\|\delta\|=\|\gamma\|$. Setting $\gamma=\alpha$, we obtain (7.1) as a consequence of Theorem 1.4. Next, for $k \in\left\{1, \ldots, d^{\prime}\right\}$ observe that $A_{k}^{(h)} B^{(N) \alpha} K_{t}^{\prime}(g ; h)$ is the kernel of the operator

$$
(-1) B^{(N) \alpha} S_{t} A_{k}=B^{(N) \alpha} S_{t / 2}\left(A_{k} S_{t / 2}^{*}\right)^{*} .
$$

By Theorem 1.4 applied to $H^{*}$, the kernel of $A_{k} S_{t / 2}^{*}$ satisfies global Gaussian bounds (with an extra $t^{-1 / 2}$ factor). Combining with (7.1) we deduce an estimate

$$
\left|A_{k}^{(h)} B^{(N) \alpha} K_{t}^{\prime}(g ; h)\right| \leq c t^{-(\|\alpha\|+1) / 2} t^{-D / 2} e^{-b d_{a}(g ; h)^{2} / t}
$$

for all $t \geq 1, g, h \in G$, and (7.2) follows in a straightforward way.

Reasoning as in the proof of Proposition 4.1, we can write

$$
A_{k} B^{(N) \alpha}=B^{(N) \alpha} A_{k}+\left[A_{k}, B^{(N) \alpha}\right]=B^{(N) \alpha} A_{k}+\sum_{\beta} \sigma_{k, \beta}^{\prime} B^{(N) \beta},
$$

where $\sigma_{k, \beta}^{\prime} \in \mathcal{E}$ and the sum is over $\beta \in J(\mathfrak{n})$ with $|\beta|=|\alpha|$ and $\|\beta\| \geq$ $\|\alpha\|+1$. Then applying Theorem 1.4 gives that

$$
\left|A_{k}^{(g)} B^{(N) \alpha} K_{t}^{\prime}(g ; h)\right| \leq c t^{-(\|\alpha\|+1) / 2} t^{-D / 2} e^{-b d_{a}(g ; h)^{2} / t}
$$

for all $t \geq 1, g, h \in G$, and (7.3) follows.

The kernel $K^{(\alpha)}$ of $R_{\alpha}$ is given by

$$
K^{(\alpha)}(g ; h)=\frac{1}{\Gamma(\|\alpha\| / 2)} \int_{1}^{\infty} d t t^{(\|\alpha\| / 2)-1} B^{(N) \alpha} K_{t}^{\prime}(g ; h)
$$

It is routine to verify from (7.1)-(7.3) that $K^{(\alpha)}$ satisfies standard CalderónZygmund estimates with respect to the distance $d_{a}$, with a singularity only at infinity. 
More precisely

$$
\left|K^{(\alpha)}(g ; h)\right| \leq c\left(1+d_{a}(g ; h)\right)^{-D}
$$

and

$\left|K^{(\alpha)}(g ; h)-K^{(\alpha)}(g ; l)\right|+\left|K^{(\alpha)}(h ; g)-K^{(\alpha)}(l ; g)\right| \leq c d_{a}(h ; l)\left(1+d_{a}(g ; h)\right)^{-(D+1)}$

for all $g, h, l \in G$ such that $d_{a}(h ; l) \leq(1 / 2) d_{a}(g ; h)$. Then by standard singular integration theory (see for example [8]), to prove Proposition 7.1 it is enough to prove that $R_{\alpha}$ is bounded in $L_{2}$. This we achieve by an almost-orthogonal decomposition, whose form follows [2, Section 25].

We write $R_{\alpha}=\sum_{j=1}^{\infty} R_{j}$, where $R_{j}$ has kernel $K_{(j)}$ defined by

$$
K_{(j)}(g ; h)=\frac{1}{\Gamma(\|\alpha\| / 2)} \int_{2^{j-1}}^{2^{j}} d t t^{(\|\alpha\| / 2)-1} B^{(N) \alpha} K_{t}^{\prime}(g ; h)
$$

It is easy to see that

$$
\int d g K_{(j)}(g ; h)=\int d g K_{(j)}(h ; g)=0
$$

and from (7.1)-(7.3) one derives the estimates

$$
\begin{gathered}
\int d g\left|K_{(j)}(g ; h)\right|+\int d g\left|K_{(j)}(h ; g)\right| \leq c \\
\int d g\left|K_{(j)}(g ; h)-K(j)(g ; l)\right|+\int d g\left|K_{(j)}(h ; g)-K_{(j)}(l ; g)\right| \\
\leq c 2^{-j / 2} d_{a}(h ; l) \\
\int d g\left|K_{(j)}(g ; h)\right| d_{a}(g ; h)+\int d g\left|K_{(j)}(h ; g)\right| d_{a}(g ; h) \leq c 2^{j / 2}
\end{gathered}
$$

for all $j \in \mathbb{N}$ and $h, l \in G$. Let $K_{j, k}$ be the kernel of the operator $R_{j}^{*} R_{k}$. In case $j \leq k$ we argue in a standard way using (7.4)-(7.7) to get the estimate

$$
\begin{aligned}
\int d h\left|K_{j, k}(g ; h)\right| & \leq \int d l\left|K_{(j)}(l ; g)\right|\left\{\int d h\left|K_{(k)}(l ; h)-K_{(k)}(g ; h)\right|\right\} \\
& \leq c^{\prime} 2^{-(k-j) / 2}
\end{aligned}
$$

(for a similar argument, see $\left[12\right.$, Section III]). It follows that $\left\|R_{j}^{*} R_{k}\right\|_{\infty \rightarrow \infty} \leq$ $c^{\prime} 2^{-(k-j) / 2}$. On the other hand, (7.5) implies that

$$
\left\|R_{j}^{*} R_{k}\right\|_{1 \rightarrow 1} \leq\left\|R_{j}^{*}\right\|_{1 \rightarrow 1}\left\|R_{k}\right\|_{1 \rightarrow 1} \leq c^{2} .
$$


By interpolation we obtain $\left\|R_{j}^{*} R_{k}\right\|_{2 \rightarrow 2} \leq c^{\prime \prime} 2^{-(k-j) / 4}$ for $j \leq k$. Because $R_{k}^{*} R_{j}=\left(R_{j}^{*} R_{k}\right)^{*}$ it follows that

$$
\left\|R_{j}^{*} R_{k}\right\|_{2 \rightarrow 2} \leq c^{\prime \prime} 2^{-|j-k| / 4}
$$

for all $j, k \in \mathbb{N}$. A repetition of these arguments yields

$$
\left\|R_{j} R_{k}^{*}\right\|_{2 \rightarrow 2} \leq c^{\prime \prime} 2^{-|j-k| / 4} .
$$

Applying the Cotlar-Stein lemma and routine arguments, we deduce that $R_{\alpha}=\sum_{j} R_{j}$ is bounded in $L_{2}$. This concludes the proof of the proposition.

Let $Y_{1}, \ldots, Y_{m}$ and $w=j_{1}+\cdots+j_{m}$ be as in the statement of Theorem 1.5. As remarked after Corollary 1.3, there are $w_{i} \in \mathfrak{n} \cap \mathfrak{q}_{N ; j_{i}}$ such that $Y_{1} \cdots Y_{m}$ equals $W_{1} \cdots W_{m}$ modulo terms of form $P Z Q$, where $W_{i}=$ $d L_{G}\left(w_{i}\right), P, Q \in \mathcal{R}(G), Z=d L_{G}(z)$ for some $z \in \mathfrak{s}$. Using an expression (4.8) for $W_{1} \cdots W_{m}$ we have

$$
Y_{1} \cdots Y_{m}=\sum_{\alpha} \sigma_{\alpha} B^{(N) \alpha}+\sum P Z Q
$$

with $\sigma_{\alpha} \in \mathcal{E}$ and summation over $\alpha \in J(\mathfrak{n})$ such that $|\alpha|=m,\|\alpha\| \geq w$. It easily follows from Theorem 1.2 that the operators $P Z Q H^{-w / 2, \infty}$ are bounded in $L_{1}$ and in $L_{\infty}$, hence in $L_{q}$ for all $q \in[1, \infty]$. Similarly, if $\|\alpha\|>w$ it follows from (7.1) that $B^{(N) \alpha} H^{-w / 2, \infty}$ is bounded in $L_{q}$ for all $q \in[1, \infty]$. If $\|\alpha\|=w$ we apply Proposition 7.1. Thus $Y_{1} \cdots Y_{m} H^{-w / 2, \infty}$ is bounded in $L_{q}, 1<q<\infty$, and from $L_{1}$ to weak- $L_{1}$.

\section{Proof of Theorem 1.6}

In this section we assume that the kernel $K_{t}$ satisfies global Gaussian bounds (1.4). To prove Theorem 1.6 we exploit a non-nilpotency property, given in Lemma 2.9 above, of elements of $\mathfrak{g}-\mathfrak{n}^{\prime}$. The details are as follows.

Fix $x \in \mathfrak{g}-\mathfrak{n}^{\prime}$. By Lemma 2.9 there exists $n \in \mathfrak{n}$ such that $(\operatorname{ad} x)^{j} n \notin \mathfrak{q}_{N ; 2}$ for all $j \in \mathbb{N}$. Set $y_{j}=(\operatorname{ad} x)^{j+1} n$ for all $j \in \mathbb{N}_{0}$. Note that $y_{j} \notin \mathfrak{q}_{N ; 2}$ and $y_{j} \in \mathfrak{g}_{x} \cap \mathfrak{n}$ (where $\mathfrak{g}_{x}$ is the smallest ideal of $\mathfrak{g}$ containing $x$ ).

Set $X=d L_{G}(x), Y_{j}=d L_{G}\left(y_{j}\right)$ and write $(\operatorname{ad} X) Z=[X, Z]$ for $Z \in$ $\mathcal{R}(G)$. To establish Theorem 1.6, it suffices to obtain for each $j \in \mathbb{N}$ an estimate

$$
\left\|X^{j} Y_{0} S_{t}\right\|_{2 \rightarrow 2} \geq c(j) t^{-1 / 2}
$$

for all $t \geq 1$, where $c(j)>0$. 
The key is the commutator identity

$$
\begin{aligned}
X^{j} Y_{0} & =(\operatorname{ad} X)^{j} Y_{0}+\sum_{m=0}^{j-1} c_{j m}\left((\operatorname{ad} X)^{m} Y_{0}\right) X^{j-m} \\
& =Y_{j}+\sum_{m=0}^{j-1} c_{j m} Y_{m} X^{j-m},
\end{aligned}
$$

where $c_{j m}$ are universal constants; this identity is easily derived by induction on $j \in \mathbb{N}$. To establish (8.1) we show that, for large $t,\left\|Y_{j} S_{t}\right\|_{2 \rightarrow 2} \geq c t^{-1 / 2}$ while $\left\|Y_{m} X^{j-m} S_{t}\right\| \leq c^{\prime} t^{-3 / 4}$.

We need the following estimate: for each $j \in \mathbb{N}_{0}$ there is $c_{j}^{\prime}>0$ such that

$$
\left|\exp \left(s y_{j}\right)\right|_{a} \geq c_{j}^{\prime} s
$$

for all $s \geq 1$, with $\exp$ the exponential map of $G$. This is a consequence of the fact that $y_{j} \in \mathfrak{n}-\mathfrak{q}_{N ; 2}$, and can be justified as follows. Since $y_{j} \in \mathfrak{n}$ we have $\tau\left(y_{j}\right)=0$, and then it follows from part (V) of Lemma 10.6 that $\exp \left(s y_{j}\right)=\exp _{G_{N}}\left(s y_{j}\right)=\exp _{Q_{N}}\left(s y_{j}\right)$ for all $s \in \mathbb{R}$. Moreover by Lemma 2.14 we may replace the modulus $|\cdot|_{a}$ in (8.3) with the $Q_{N}$-modulus $|\cdot|_{Q_{N}}$. Thus we are reduced to proving $\left|\exp _{Q_{N}}\left(s y_{j}\right)\right|_{Q_{N}} \geq c_{j}^{\prime \prime} s, s \geq 1 ;$ since $y_{j} \in \mathfrak{q}_{N}-\mathfrak{q}_{N ; 2}$, this is a consequence of well-known estimates for the modulus on the simply connected nilpotent group $Q_{N}$ (see Section IV.5 of [35]). Thus (8.3) holds.

Therefore we can apply the following result with $y=y_{j}$.

Proposition 8.1 Suppose the kernel $K_{t}$ corresponding to $H$ satisfies Gaussian bounds (1.4). Let $y \in \mathfrak{g}$ and set $Y=d L_{G}(y)$.

(I) There exist $\kappa, c>0$ such that

$$
\left\|Y S_{t}\right\|_{2 \rightarrow 2} \geq c s^{-1}
$$

for all $t, s>0$ such that $|\exp (s y)|_{a}^{2} \geq \kappa t$.

(II) If there exists $c_{1}>0$ such that $|\exp (s y)|_{a} \geq c_{1} s$ for all $s \geq 1$, then there exists $c_{2}>0$ such that

$$
\left\|Y S_{t}\right\|_{2 \rightarrow 2} \geq c_{2} t^{-1 / 2}
$$

for all $t \geq 1$.

Proof. Let $K_{t}^{*}(g)=\overline{K_{t}\left(g^{-1}\right)}$ denote the kernel of the adjoint semigroup $S_{t}^{*}$, and define a positive-definite function $W_{t}$ as the convolution $W_{t}=K_{t} * K_{t}^{*}$ for all $t>0$. The arguments of [13, Section 2] or [19] show that there is $c_{1}>0$ such that

$$
W_{t}(e) \geq c_{1} V_{a}(t)^{-1 / 2}
$$


for all $t>0$. Moreover, it is easy to argue that $W_{t}$ satisfies global Gaussian bounds

$$
\left|W_{t}(g)\right| \leq c^{\prime} V_{a}(t)^{-1 / 2} e^{-b^{\prime}|g|_{a}^{2} / t}
$$

for all $t>0, g \in G$. Hence one can choose $\kappa>0$ such that

$$
\left|W_{t}(g)\right| \leq 2^{-1} c_{1} V_{a}(t)^{-1 / 2}
$$

for all $t>0, g \in G$ satisfying the condition $|g|_{a}^{2} \geq \kappa t$, where $c_{1}$ is as in (8.4). Combining with (8.4) we deduce that

$$
\left|W_{t}(e)-W_{t}(g)\right| \geq 2^{-1} c_{1} V_{a}(t)^{-1 / 2}
$$

whenever $|g|_{a}^{2} \geq \kappa t$. Now set $g=\exp (s y)$ and note that $\frac{d}{d u} W_{t}(\exp u y)=$ $-\left(Y W_{t}\right)(\exp u y)$ to obtain

$2^{-1} c_{1} V_{a}(t)^{-1 / 2} \leq\left|W_{t}(e)-W_{t}(\exp s y)\right| \leq \int_{0}^{s} d u\left|\left(Y W_{t}\right)(\exp u y)\right| \leq s\left\|Y W_{t}\right\|_{\infty}$ for all $t, s>0$ satisfying $|\exp (s y)|_{a}^{2} \geq \kappa t$. Observing that $Y W_{t}=\left(Y K_{t}\right) *$ $K_{t}^{*}=\left(Y S_{t / 2} K_{t / 2}\right) * K_{t}^{*}$, we have

$$
\left\|Y W_{t}\right\|_{\infty} \leq\left\|Y S_{t / 2}\right\|_{2 \rightarrow 2}\left\|K_{t / 2}\right\|_{2}\left\|K_{t}^{*}\right\|_{2} \leq c V_{a}(t)^{-1 / 2}\left\|Y S_{t / 2}\right\|_{2 \rightarrow 2}
$$

for all $t>0$ (the estimate $\left\|K_{t}^{*}\right\|_{2}=\left\|K_{t}\right\|_{2} \leq c V_{a}(t)^{-1 / 4}$ is a consequence of (1.4)). Part (I) of the proposition follows immediately from the previous two displayed inequalities.

It suffices to prove the estimate of part (II) for all $t \geq T$ for some $T>0$, since

$$
\left\|Y S_{t_{2}}\right\|_{2 \rightarrow 2} \leq\left\|Y S_{t_{1}}\right\|_{2 \rightarrow 2}\left\|S_{t_{2}-t_{1}}\right\|_{2 \rightarrow 2} \leq\left\|Y S_{t_{1}}\right\|_{2 \rightarrow 2}
$$

whenever $t_{2}>t_{1}>0$. Let $\kappa$ be as in the statement of part (I) and set

$$
t(s)=\kappa^{-1}|\exp (s y)|_{a}^{2} .
$$

Then part (I) and the assumption of part (II) give

$$
\left\|Y S_{t(s)}\right\|_{2 \rightarrow 2} \geq c s^{-1} \geq c^{\prime} t(s)^{-1 / 2}
$$

for all $s \geq 1$. Since $\{t(s): s \geq 1\}$ contains an interval $[T, \infty\rangle$ for some $T>0$, the proposition follows.

From (8.3) and Proposition 8.1, for each $j \in \mathbb{N}_{0}$ there is $c_{j}>0$ such that

$$
\left\|Y_{j} S_{t}\right\|_{2 \rightarrow 2} \geq c_{j} t^{-1 / 2}
$$

for all $t \geq 1$. The next proposition yields an estimate for the terms $Y_{m} X^{j-m} S_{t}$ coming from (8.2). Let $\mathcal{R}^{+}(G)$ denote the subspace of $\mathcal{R}(G)$ spanned by all $d L_{G}\left(x_{1}\right) \cdots d L_{G}\left(x_{m}\right), m \in \mathbb{N}, x_{1}, \ldots, x_{m} \in \mathfrak{g}$; it is an ideal of codimension one in $\mathcal{R}(G)$. Note that $\mathcal{R}^{+}(G)$ is spanned by all $A^{\alpha}$ with $\alpha \in J\left(d^{\prime}\right)$ and $|\alpha| \geq 1$. 
Proposition 8.2 If $N=d L_{G}(n), n \in \mathfrak{n}$, and $P \in \mathcal{R}^{+}(G)$, then there is $c>0$ such that

$$
\left\|N P S_{t}\right\|_{2 \rightarrow 2} \leq c t^{-3 / 4} \quad \text { for all } t \geq 1 .
$$

Remark The factor $t^{-3 / 4}$ can be improved to $t^{-1}$ (this is a special case of Remark (e) in Section 1), but this is not necessary for our current purposes.

The proof of Proposition 8.2 requires a preliminary lemma (whose statement remains valid if $\mathfrak{n}$ is replaced by an arbitrary ideal of $\mathfrak{g}$ ).

Lemma 8.3 If $N=d L_{G}(n), n \in \mathfrak{n}$, and $P \in \mathcal{R}(G)$, then $N P \in \mathcal{R}(G)$ can be written as a finite sum

$$
N P=\sum_{i} P_{i} N_{i}
$$

where $P_{i} \in \mathcal{R}(G)$ and $N_{i}=d L_{G}\left(n_{i}\right), n_{i} \in \mathfrak{n}$.

Proof. It suffices to prove it in the case $P=X_{1} \cdots X_{m}$ where $m \in \mathbb{N}$, $X_{j}=d L_{G}\left(x_{j}\right), x_{j} \in \mathfrak{g}$. Since $\mathfrak{n}$ is an ideal of $\mathfrak{g}$ we have $n^{\prime \prime}=\left[x_{1}, n\right] \in \mathfrak{n}$. Setting $N^{\prime \prime}=d L_{G}\left(n^{\prime \prime}\right)$ we note that

$$
N X_{1} \cdots X_{m}=-N^{\prime \prime} X_{2} \cdots X_{m}+X_{1} N X_{2} \cdots X_{m}
$$

and the lemma is easily derived by induction on $m$.

Continuing with the proof of Proposition 8.2, it suffices to prove the estimate with $P=Q A_{k}$ for some $Q \in \mathcal{R}(G), k \in\left\{1, \ldots, d^{\prime}\right\}$. Then for $\varphi \in L_{2 ; \infty}$ we have

$$
\|N P \varphi\|_{2}^{2}=-\left(P \varphi, N^{2} Q A_{k} \varphi\right),
$$

and Lemma 8.3 yields that

$$
N^{2} Q=N\left(\sum_{i} Q_{i} N_{i}\right)=\sum_{i, j} Q_{i j} M_{j} N_{i}
$$

for some $Q_{i}, Q_{i j} \in \mathcal{R}(G)$ and $N_{i}=d L_{G}\left(n_{i}\right), M_{j}=d L_{G}\left(m_{j}\right), n_{i}, m_{j} \in \mathfrak{n}$. Thus

$$
\|N P \varphi\|_{2}^{2} \leq \sum_{i, j}\left\|Q_{i j}^{*} P \varphi\right\|_{2}\left\|M_{j} N_{i} A_{k} \varphi\right\|_{2}
$$

where $Q_{i j}^{*}$ denotes the formal adjoint of $Q_{i j}$ in $\mathcal{R}(G)$. Set $\varphi=S_{t} \psi$ where $\psi \in L_{2}$, and note from Corollary 3.2 that $\left\|Q_{i j}^{*} P S_{t}\right\|_{2 \rightarrow 2} \leq c$ for all $t \geq 1$. Therefore applying Theorem 1.1 we get

$$
\left\|N P S_{t} \psi\right\|_{2}^{2} \leq c t^{-3 / 2}\|\psi\|_{2}^{2}
$$

for all $t \geq 1, \psi \in L_{2}$, and the proposition follows. 
Proposition 8.2 yields for each $m \in \mathbb{N}_{0}$ and $q \in \mathbb{N}$ an estimate

$$
\left\|Y_{m} X^{q} S_{t}\right\|_{2 \rightarrow 2} \leq c(m, q) t^{-3 / 4}
$$

for all $t \geq 1$. Combining (8.2), (8.5), and (8.6) we see that an estimate (8.1) holds for all sufficiently large $t$. Since the function $t \mapsto\left\|X^{j} Y_{0} S_{t}\right\|_{2 \rightarrow 2}$ is nonincreasing (cf. the proof of Proposition 8.1(II)), this suffices to give an estimate of the form (8.1) for all $t \geq 1$. The proof of Theorem 1.6 is complete.

\section{Extensions}

We briefly describe two extensions of our results: to subelliptic systems, and to non-simply connected groups.

\subsection{Subelliptic systems}

It is of interest that Theorem 1.1 extends to certain $s \times s$ subelliptic systems, where $s \in \mathbb{N}$.

Observe that $A_{k}$ extends naturally to an operator in the space $L_{2}\left(G ; \mathbb{C}^{s}\right)$ of $\mathbb{C}^{s}$-valued $L_{2}$ functions. Consider an operator $H=-\sum_{k, l=1}^{d^{\prime}} A_{k} c_{k l} A_{l}$, with each $c_{k l}$ an $s \times s$ matrix of complex constants: $c_{k l}$ acts on elements of $L_{2}\left(G ; \mathbb{C}^{s}\right)$ (regarded as row vectors) by matrix multiplication. As before, $H$ is precisely defined as the maximal accretive operator in $L_{2}\left(G ; \mathbb{C}^{s}\right)$ associated with the quadratic form $q_{H}(\varphi)=\sum_{k, l}\left(c_{k l} A_{l} \varphi, A_{k} \varphi\right)$, assuming that $q_{H}$ satisfies a Gårding inequality (1.2).

Then the estimates of Theorem 1.1 are valid for $H$, at least when $G$ has stratified nilshadow. One needs the following minor change in the proof of Proposition 4.1. The identity (4.1) (with $P=D_{t}=L^{\prime}(t)^{2} B^{(N) \alpha}$ ) is no longer valid, but can be replaced by

$$
\begin{aligned}
D_{t} \varphi= & \left(I+H_{\varepsilon}\right)^{-1} D_{t}\left(I+H_{\varepsilon}\right) \varphi \\
& -\sum_{k, l}\left(I+H_{\varepsilon}\right)^{-1}\left[A_{k, \varepsilon}, D_{t}\right] c_{k l} A_{l, \varepsilon} \varphi-\sum_{k, l}\left(I+H_{\varepsilon}\right)^{-1} A_{k, \varepsilon} c_{k l}\left[A_{l, \varepsilon}, D_{t}\right] \varphi
\end{aligned}
$$

after noting that $D_{t}$ commutes with $c_{k l}$. Note also that we do not need local regularity estimates for $H$ for the proof of Theorem 1.1: see Remark (a) in Subsection 4.1.

Finally, let us note that if $G$ is stratified nilpotent and $a_{1}, \ldots, a_{d^{\prime}}$ is a basis for $\mathfrak{h}_{1}$ then Remark (b) in Subsection 4.1 applies also to systems. In particular, one has an estimate of the form (4.9) for each $\alpha \in J(d)$, and one can argue that the semigroup $e^{-t H}$ has a smooth kernel satisying global Gaussian bounds. Details are left to the reader. 


\subsection{Non-simply connected groups}

Theorems 1.1 to 1.6 are actually valid for any connected Lie group $G$ of polynomial growth, without the assumption of simple connectedness. For completeness, we outline here the extension of our arguments to the nonsimply connected case.

For Theorems 1.1 to 1.3 and Theorem 1.5, no changes in the proofs are required. In particular, remark that in the transference argument of Subsection 4.2 we used simple connectedness of $\widetilde{G}$, but not of $G$, to obtain the homomorphism $\Lambda: \widetilde{G} \rightarrow G$.

Some changes are required, however, in the proofs of Theorems 1.4 and 1.6. Before outlining these, we briefly sketch the structure theory of $G$ : our exposition relies on the detailed results obtained in [1, Section 3], and we refer to that paper for further details.

We may assume that $G=G^{\prime} / \Gamma$ where $G^{\prime}$ is the simply connected universal covering group of $G$ and $\Gamma$ is a discrete central subgroup of $G^{\prime}$ : the Lie algebras of $G, G^{\prime}$ are identified (= $\mathfrak{g}$, say). Let $G_{N}^{\prime}$ denote the shadow of the simply connected group $G^{\prime}$, with Lie algebra $\mathfrak{g}_{N}$, and with $G^{\prime}=G_{N}^{\prime}$ identified as manifolds (we often use primes to indicate objects associated with the group $\left.G^{\prime}\right)$. Let $\tau: \mathfrak{g}_{N} \rightarrow \mathcal{L}\left(\mathfrak{g}_{N}\right), \bar{T}^{\prime}: G_{N}^{\prime} \rightarrow \operatorname{Aut}\left(\mathfrak{g}_{N}\right), T^{\prime}: G_{N}^{\prime} \rightarrow \operatorname{Aut}\left(G_{N}^{\prime}\right)$ denote the corresponding homomorphisms, as defined in Section 2.

From the properties of the group $\Gamma$ given in Section 3.2 of [1], it is straightforward to deduce that

$$
\bar{T}^{\prime}(\gamma) x=x \quad, \quad T^{\prime}(\gamma) g^{\prime}=g^{\prime} \quad, \quad T^{\prime}\left(g^{\prime}\right) \gamma=\gamma
$$

for all $\gamma \in \Gamma, g^{\prime} \in G^{\prime}$ and $x \in \mathfrak{g}$. It follows from (9.1) and the multiplication law (2.5) (with $T$ replaced by $T^{\prime}$ ), that

$$
\gamma *_{N} g^{\prime}=\gamma g^{\prime}=g^{\prime} \gamma=g^{\prime} *_{N} \gamma
$$

for all $\gamma \in \Gamma, g^{\prime} \in G^{\prime}$. Hence $\Gamma$ is a central subgroup of $G_{N}^{\prime}$, and the quotient $G_{N}=G_{N}^{\prime} / \Gamma$ is a Lie group with Lie algebra $\mathfrak{g}_{N}$. Observe that $G_{N}$ is identical as a manifold with $G=G^{\prime} / \Gamma$, and that the natural homomorphisms $G^{\prime} \rightarrow G$, $G_{N}^{\prime} \rightarrow G_{N}$ are identical as set maps: let us call this map $\Lambda$. It follows from (9.1) that $T^{\prime}$ induces a homomorphism $T: G_{N} \rightarrow \operatorname{Aut}\left(G_{N}\right)$ such that

$$
T\left(\Lambda g^{\prime}\right)\left(\Lambda h^{\prime}\right)=\Lambda\left(T^{\prime}\left(g^{\prime}\right) h^{\prime}\right)
$$

for all $g^{\prime}, h^{\prime} \in G^{\prime}$. Then $T(T(g) h)=T(h)$ for $g, h \in G$, and the multiplications in $G$ and $G_{N}$ are related by the formula (2.5).

Again from (9.1) it follows that $\bar{T}^{\prime}$ induces a homomorphism $\bar{T}: G_{N} \rightarrow$ Aut $\left(\mathfrak{g}_{N}\right)$ such that $\bar{T}\left(\Lambda g^{\prime}\right)=\bar{T}^{\prime}\left(g^{\prime}\right)$ for all $g^{\prime} \in G^{\prime}$. Then the vector fields on $G$ and $G_{N}$ are related by the formulae of Lemma 2.12 . 
As in the simply connected case, let $M, Q_{N}$ denote the analytic subgroups of $G_{N}$ with respective Lie algebras $\mathfrak{m}, \mathfrak{q}_{N}$. Then $M, Q_{N}$ are closed normal subgroups of $G_{N}$ such that $M$ commutes with $Q_{N}$, and $G_{N}=$ $M *_{N} Q_{N}$, but the intersection $\Gamma_{2}:=M \cap Q_{N}$ is a possibly nontrivial, finite central subgroup of $G_{N}$.

Proof of Theorem 1.4. The only change required is in the definition of $\eta_{R}$ in the proof of Lemma 6.2, and is due to the fact that $\Gamma_{2}=M \cap Q_{N}$ may be nontrivial. We now set

$$
\eta_{R}^{\prime \prime}\left(m *_{N} q\right)=\frac{1}{\left|\Gamma_{2}\right|} \sum_{y \in \Gamma_{2}} \eta_{R}^{\prime}\left(y *_{N} q\right)
$$

for all $m \in M, q \in Q_{N}$, where $\left|\Gamma_{2}\right|<\infty$ is the cardinality of $\Gamma_{2}$. It is easily verified that $\eta_{R}^{\prime \prime}$ is a well-defined function on $G_{N}$ and that it satisfies the properties of Lemma 6.2 for all sufficiently large $R$, say for $R \geq R_{0}$. (The restriction to large $R$ is necessary, since it might happen that $\eta_{R}^{\prime \prime}(e)<1$ for $R$ close to 1.) We complete the proof of Lemma 6.2 by defining $\eta_{R}=\eta_{R}^{\prime \prime}$ for $R \geq R_{0}$ and $\eta_{R}=\eta_{R_{0}}^{\prime \prime}$ for $1 \leq R \leq R_{0}$.

Proof of Theorem 1.6. Let the elements $x$ and $y_{j}=(\operatorname{ad} x)^{j+1} n, j \in \mathbb{N}_{0}$, be as in the proof of this theorem. The only change required is to justify the estimate (8.3) for non-simply connected $G$. Again it reduces to showing that $\left|\exp _{Q_{N}}\left(s y_{j}\right)\right|_{Q_{N}} \geq c s, s \geq 1$, where $|\cdot|_{Q_{N}}$ is the modulus on $Q_{N}$ corresponding to $b_{1}, \ldots, b_{d_{1}}$.

Define a subalgebra of $\mathfrak{q}_{N}$ by $\mathfrak{q}_{0}=\{u \in \mathfrak{q}: \tau(y) u=0$ for all $y \in \mathfrak{g}\}$. As in the proof of Lemma 2.9 we have $(\operatorname{ad} x) y_{j}-\tau(x) y_{j} \in \mathfrak{q}_{N ; 2}$, and since $(\operatorname{ad} x) y_{j} \notin \mathfrak{q}_{N ; 2}$ it follows that $\tau(x) y_{j} \notin \mathfrak{q}_{N ; 2}$. Since $\tau(x)\left(\mathfrak{q}_{0}+\mathfrak{q}_{N ; 2}\right) \subseteq \mathfrak{q}_{N ; 2}$, we deduce that $y_{j} \notin \mathfrak{q}_{0}+\mathfrak{q}_{N ; 2}$.

Let $Q_{\mathfrak{t}}$ be the unique maximal torus in the nilpotent group $Q_{N}$, and $\mathfrak{t}$ be its Lie algebra. Then $Q_{\mathfrak{t}}$ is contained in the centre of $Q_{N}$, and the quotient group $\bar{Q}_{N}=Q_{N} / Q_{\mathfrak{t}}$ is simply connected and nilpotent (see IV.1.5 in [35]). Moreover, it easily follows from results of Sections $3.2,3.3$ of [1] that $\mathfrak{t} \subseteq \mathfrak{q}_{0}$.

Combining the above remarks, we obtain (8.3) as a consequence of the following lemma for the nilpotent group $Q_{N}$.

Lemma 9.1 If $y \in \mathfrak{q}_{N}, y \notin \mathfrak{t}+\mathfrak{q}_{N ; 2}$ then there is $c>0$ with

$$
\left|\exp _{Q_{N}}(s y)\right|_{Q_{N}} \geq c s \quad \text { for all } s \geq 1 \text {. }
$$

Proof. Consider the quotient $\bar{Q}_{N}=Q_{N} / Q_{\mathfrak{t}}$. Note that if $\pi: \mathfrak{q}_{N} \rightarrow \overline{\mathfrak{q}}_{N}=$ $\mathfrak{q}_{N} / \mathfrak{t}$ denotes the corresponding Lie algebra homomorphism, and $\overline{\mathfrak{q}}_{N ; 2}$ is the commutator ideal of $\overline{\mathfrak{q}}_{N}$, then $\pi y \notin \overline{\mathfrak{q}}_{N ; 2}$. 
If $|\cdot|$ is a modulus on $\bar{Q}_{N}$ then modulus estimates for a simply connected nilpotent group ([35, Section IV.5]) yield $\left|\exp _{\bar{Q}_{N}}(s \pi y)\right| \geq c s$ for all $s \geq 1$. But it is elementary (e.g., Lemma 3.1 of [23]) that $\left|\exp _{Q_{N}}(s y)\right|_{Q_{N}} \geq$ $c\left|\exp _{\bar{Q}_{N}}(s \pi y)\right|$ for $s \geq 1$. This completes the proof of the lemma, and the proof of Theorem 1.6 for non-simply connected groups.

\section{Appendix}

In this Appendix, we prove a number of algebraic results which are used in the main text.

\subsection{Invariance of the $\mathfrak{q}_{N ; j}$}

We show in this subsection that the nilshadow $\mathfrak{q}_{N}$ is uniquely defined up to isomorphism, and that the subspaces $\mathfrak{q}_{N ; j}$ in the lower central series are invariants, and characteristic ideals, of $\mathfrak{g}$.

First we reformulate the definition of the nilshadow in terms of Cartan subalgebras of $\mathfrak{q}$. Let $\mathfrak{v}$ satisfy properties (I) and (II) of Lemma 2.1, and consider the associated nilshadow $\mathfrak{q}_{N}=\left(\mathfrak{q},[\cdot, \cdot]_{N}\right)$ defined by $(2.3)$.

Lemma 10.1 Given $\mathfrak{v}$ as above, there is a unique Cartan subalgebra $\mathfrak{h}=$ $\mathfrak{h}(\mathfrak{v})$ of $\mathfrak{q}$ with $\mathfrak{v} \subseteq \mathfrak{h}$. Then $\mathfrak{q}=\mathfrak{h}+\mathfrak{n}$ and the bracket $[\cdot, \cdot]_{N}$ is given by

$$
\left[w_{1}+n_{1}, w_{2}+n_{2}\right]_{N}=\left[w_{1}+n_{1}, w_{2}+n_{2}\right]-S\left(w_{1}\right) n_{2}+S\left(w_{2}\right) n_{1}
$$

for all $w_{1}, w_{2} \in \mathfrak{h}, n_{1}, n_{2} \in \mathfrak{n}$.

Proof. Define

$$
\mathfrak{h}=\{x \in \mathfrak{q}: S(v) x=0 \text { for all } v \in \mathfrak{v}\} \quad ;
$$

since the $S(v)$ are derivations this is a subalgebra of $\mathfrak{q}$. It follows from the properties of $\mathfrak{v}$ and Lemma 2.2 that $\mathfrak{v} \subseteq \mathfrak{h}$ and $\mathfrak{h}=\mathfrak{v} \oplus(\mathfrak{h} \cap \mathfrak{n})$. From the latter splitting and (2.3) it is easy to verify that $[x, y]_{N}=[x, y]$ for all $x, y \in \mathfrak{h}$. Therefore $\mathfrak{h}$ is nilpotent.

Suppose $y \in \mathfrak{q}$ satisfies $[y, \mathfrak{h}] \subseteq \mathfrak{h}$. Then $S(v) y \in \mathfrak{h}$ for all $v \in \mathfrak{v}$, because $\mathfrak{v} \subseteq \mathfrak{h}$ and $S(v)$ is a polynomial in ad $v$. Therefore $S(v)^{2} y=0$ and the semisimplicity of $S(v)$ implies that $S(v) y=0$. Thus $y \in \mathfrak{h}$, and we have verified that $\mathfrak{h}$ is a Cartan subalgebra of $\mathfrak{q}$.

If $\mathfrak{h}^{\prime}$ is any Cartan subalgebra of $\mathfrak{q}$ with $\mathfrak{v} \subseteq \mathfrak{h}^{\prime}$ then nilpotency of $\mathfrak{h}^{\prime}$ implies that $S(x) y=0$ for all $x, y \in \mathfrak{h}^{\prime}$ (Lemma 2.2). Taking $x \in \mathfrak{v}$ we obtain $\mathfrak{h}^{\prime} \subseteq \mathfrak{h}$, hence $\mathfrak{h}^{\prime}=\mathfrak{h}$ since all Cartan subalgebras have the same dimension. 
Finally, (10.1) follows straightforwardly from (2.3), the splitting $\mathfrak{h}=$ $\mathfrak{v} \oplus(\mathfrak{h} \cap \mathfrak{n})$, and the following observation: if $w=v+n, v \in \mathfrak{v}, n \in \mathfrak{h} \cap \mathfrak{n}$, then $S(w)=S(v)$. Indeed the mapping $y \mapsto S(y)$ is linear on $\mathfrak{h}$ by Lemma 2.2, whence $S(w)=S(v)+S(n)=S(v)$.

Let $\mathfrak{v}^{(1)}, \mathfrak{v}^{(2)}$ be subspaces of $\mathfrak{q}$ which satisfy properties (I) and (II) of Lemma 2.1. Consider the associated nilshadows $\mathfrak{q}_{N}^{(i)}=\left(\mathfrak{q},[\cdot, \cdot]_{N}^{(i)}\right)$, and the corresponding lower central series $\mathfrak{q}_{N ; j}^{(i)}, j \in \mathbb{N}, i=1,2$.

If $\Phi$ is an arbitrary automorphism of $\mathfrak{g}$ then it is straightforward to see that

$$
\Phi(S(x) y)=S(\Phi x)(\Phi y) \quad, \quad \Phi(K(x) y)=K(\Phi x)(\Phi y)
$$

for all $x, y \in \mathfrak{g}$.

Lemma 10.2 There exists $n \in \mathfrak{n}$ such that $\Phi=\left.\left(e^{\operatorname{ad} n}\right)\right|_{\mathfrak{q}} \in G L(\mathfrak{q})$ is a Lie isomorphism of $\mathfrak{q}_{N}^{(1)}$ onto $\mathfrak{q}_{N}^{(2)}$. One has equality of subspaces $\mathfrak{q}_{N ; j}^{(1)}=\mathfrak{q}_{N ; j}^{(2)}$ for all $j \in \mathbb{N}$.

Proof. Applying Lemma 10.1, let $\mathfrak{h}^{(i)}, i=1,2$, be the Cartan subalgebras of $\mathfrak{q}$ with $\mathfrak{v}^{(i)} \subseteq \mathfrak{h}^{(i)}$. A standard conjugacy result for Cartan subalgebras of solvable algebras $([4,36])$ gives the existence of $n \in \mathfrak{n}$ such that $\Phi\left(\mathfrak{h}^{(1)}\right)=\mathfrak{h}^{(2)}$ with $\Phi=\left(e^{\operatorname{ad} n}\right) \mid \mathfrak{q}$. From (10.1) and (10.2) we then obtain $\Phi\left([x, y]_{N}^{(1)}\right)=$ $[\Phi x, \Phi y]_{N}^{(2)}$ for all $x, y \in \mathfrak{q}$, proving the first statement.

Since $\mathfrak{q}_{N ; 1}^{(1)}=\mathfrak{q}=\mathfrak{q}_{N ; 1}^{(2)}$ it suffices to prove the second statement when $j \geq 2$. Then $\mathfrak{q}_{N ; j}^{(1)} \subseteq \mathfrak{n}$ by Proposition 2.3. Since $\mathfrak{q}_{N ; j}^{(1)}$ is an ideal of $\mathfrak{q}_{N}^{(1)}$ and $[x, y]_{N}^{(1)}=[x, y]$ for all $x, y \in \mathfrak{n}$, it follows that $\mathfrak{q}_{N ; j}^{(1)}$ is an ideal of $\mathfrak{n}$. Therefore $\Phi\left(\mathfrak{q}_{N ; j}^{(1)}\right)=e^{\text {adn } n}\left(\mathfrak{q}_{N ; j}^{(1)}\right)=\mathfrak{q}_{N ; j}^{(1)}$. But $\Phi\left(\mathfrak{q}_{N ; j}^{(1)}\right)=\mathfrak{q}_{N ; j}^{(2)}$ because $\Phi$ maps $\mathfrak{q}_{N}^{(1)}$ isomorphically to $\mathfrak{q}_{N}^{(2)}$, and the lemma follows.

Therefore the subspaces $\mathfrak{q}_{N ; j}$ are invariants of $\mathfrak{g}$. Finally we have

Corollary 10.3 The subspaces $\mathfrak{q}_{N ; j}$ are characteristic ideals of $\mathfrak{g}$.

Proof. Let $\mathfrak{q}_{N}$ be the nilshadow associated with the subspace $\mathfrak{v}$, and let $\Phi$ be an arbitrary automorphism of $\mathfrak{g}$. Then $\Phi(\mathfrak{q})=\mathfrak{q}, \Phi(\mathfrak{n})=\mathfrak{n}$, and the subspace $\mathfrak{v}^{\prime}=\Phi(\mathfrak{v})$ satisfies properties (I), (II) of Lemma 2.1. If $\mathfrak{q}_{N}^{\prime}$ denotes the nilshadow associated with $\mathfrak{v}^{\prime}$ then it is easy to see from (2.3) or (10.1), applying (10.2), that $\Phi$ maps $\mathfrak{q}_{N}$ isomorphically onto $\mathfrak{q}_{N}^{\prime}$. Therefore $\Phi\left(\mathfrak{q}_{N ; j}\right)=\mathfrak{q}_{N ; j}^{\prime}=\mathfrak{q}_{N ; j}$ with the second equality by Lemma 10.2. 


\subsection{The ideals $\mathfrak{s}$ and $\mathfrak{n}^{\prime}$}

Let $\mathfrak{g}$ be a Lie algebra of type $R$, with radical $\mathfrak{q}$ and nilradical $\mathfrak{n}$.

Lemma 10.4 There exists a semisimple ideal $\mathfrak{s}$ of $\mathfrak{g}$ which contains every semisimple ideal of $\mathfrak{g}$, and a nilcompact ideal $\mathfrak{n}^{\prime}$ of $\mathfrak{g}$ which contains every nilcompact ideal of $\mathfrak{g}$. Moreover, $\mathfrak{s}, \mathfrak{n}^{\prime}$ are characteristic ideals of $\mathfrak{g}$, and $\mathfrak{n}^{\prime}=\mathfrak{s} \oplus \mathfrak{n}, \mathfrak{s} \cap \mathfrak{q}=\{0\}$.

For any Levi subalgebra $\mathfrak{m}$ of $\mathfrak{g}$ one has $\mathfrak{s} \subseteq \mathfrak{m}$ and

$$
\mathfrak{s}=\{x \in \mathfrak{m}:[x, \mathfrak{q}]=\{0\}\} .
$$

Proof. Fix a Levi subalgebra $\mathfrak{m}$ and define $\mathfrak{s}$ by (10.3). One easily checks that $\mathfrak{s}$ is an ideal of $\mathfrak{m}$, and hence $\mathfrak{s}$ is semisimple because $\mathfrak{m}$ is semisimple. Then $\mathfrak{s}$ is an ideal of $\mathfrak{g}$, because $\mathfrak{g}=\mathfrak{m} \oplus \mathfrak{q}$ and $[\mathfrak{s}, \mathfrak{q}]=\{0\}$. Because $\mathfrak{s}$ is semisimple, the intersection $\mathfrak{s} \cap \mathfrak{q}$ is trivial.

Suppose that $\mathfrak{a}$ is a semisimple ideal of $\mathfrak{g}$; we show $\mathfrak{a} \subseteq \mathfrak{s}$. Let $\operatorname{Int}(\mathfrak{n})$ denote the group of automorphisms of $\mathfrak{g}$ generated by the $e^{\text {ada }}$ for $a \in \mathfrak{n}$. Since $\mathfrak{a}$ is a semisimple subalgebra of $\mathfrak{g}$, by Corollary 3.14.3 of [32] there exists $\Phi \in \operatorname{Int}(\mathfrak{n})$ such that $\Phi(\mathfrak{a}) \subseteq \mathfrak{m}$. Since $\mathfrak{a}$ is an ideal, $\Phi(\mathfrak{a})=\mathfrak{a}$ so that $\mathfrak{a} \subseteq \mathfrak{m}$. Because $\mathfrak{a}$ and $\mathfrak{q}$ are ideals we get

$$
[\mathfrak{a}, \mathfrak{q}] \subseteq \mathfrak{a} \cap \mathfrak{q} \subseteq \mathfrak{m} \cap \mathfrak{q}=\{0\}
$$

and therefore $\mathfrak{a} \subseteq \mathfrak{s}$, as desired.

Thus $\mathfrak{s}$ contains every semisimple ideal; it easily follows that $\mathfrak{s}$ is independent of the choice of Levi subalgebra $\mathfrak{m}$ used to define it, and that $\mathfrak{s}$ is a characteristic ideal of $\mathfrak{g}$.

Defining $\mathfrak{n}^{\prime}=\mathfrak{s} \oplus \mathfrak{n}$, it is clear that $\mathfrak{n}^{\prime}$ is a nilcompact ideal and a characteristic ideal of $\mathfrak{g}$. Let us show $\mathfrak{a} \subseteq \mathfrak{n}^{\prime}$ for any nilcompact ideal $\mathfrak{a}$. Write $\mathfrak{a}=\mathfrak{a}_{s} \oplus \mathfrak{a}_{n}$, where $\mathfrak{a}_{s}, \mathfrak{a}_{n}$ are respectively semisimple and nilpotent ideals of $\mathfrak{a}$. It is easy to see that $\mathfrak{a}_{n}$ is the radical (or nilradical) of $\mathfrak{a}$, and $\mathfrak{a}_{s}$ contains every semisimple ideal of $\mathfrak{a}$. Therefore $\mathfrak{a}_{s}, \mathfrak{a}_{n}$ are characteristic ideals of $\mathfrak{a}$, and hence $\mathfrak{a}_{s}, \mathfrak{a}_{n}$ are ideals of $\mathfrak{g}$. Since $\mathfrak{s}, \mathfrak{n}$ are respectively the largest semisimple and nilpotent ideals of $\mathfrak{g}$, we get $\mathfrak{a}_{s} \subseteq \mathfrak{s}$ and $\mathfrak{a}_{n} \subseteq \mathfrak{n}$. Then $\mathfrak{a} \subseteq \mathfrak{n}^{\prime}$ as desired.

\subsection{Twisted multiplications on Lie groups}

First we consider a twisting construction for Lie algebras.

Lemma 10.5 Let $\mathfrak{a}$ be a Lie algebra with Lie bracket $[\cdot, \cdot]$, and let $\tau: \mathfrak{a} \rightarrow$ $\mathcal{L}(\mathfrak{a})$ be a representation of $\mathfrak{a}$ in itself by derivations. If

$$
\tau(\tau(x) y)=0
$$


for all $x, y \in \mathfrak{a}$, then

$$
[x, y]^{\prime}=[x, y]+\tau(x) y-\tau(y) x
$$

defines a Lie bracket $[\cdot, \cdot]^{\prime}$ on the vector space $\mathfrak{a}$.

Proof. To check the Jacobi identity for $[\cdot, \cdot]^{\prime}$ is a straightforward computation using (10.4) and the representation and derivation properties.

The following two examples illustrate the generality of this lemma.

(1) Let $\mathfrak{a}_{1}, \mathfrak{a}_{2}$ be Lie algebras and $\tau_{0}: \mathfrak{a}_{1} \rightarrow \mathcal{L}\left(\mathfrak{a}_{2}\right)$ a representation of $\mathfrak{a}_{1}$ in $\mathfrak{a}_{2}$ by derivations. Consider the direct sum algebra $\mathfrak{a}=\mathfrak{a}_{1} \times \mathfrak{a}_{2}$ and $\tau: \mathfrak{a} \rightarrow \mathcal{L}(\mathfrak{a})$ defined by

$$
\tau\left(x_{1}, x_{2}\right)\left(y_{1}, y_{2}\right)=\left(0, \tau_{0}\left(x_{1}\right) y_{2}\right)
$$

for $x_{i}, y_{i} \in \mathfrak{a}_{i}, i=1,2$. Then $\tau$ satisfies the hypotheses of Lemma 10.5 and the algebra $\mathfrak{a}^{\prime}=\left(\mathfrak{a},[\cdot, \cdot]^{\prime}\right)$ is (isomorphic to) the semidirect product $\mathfrak{a}_{1} \times_{\tau_{0}} \mathfrak{a}_{2}$.

(2) Let $\mathfrak{a}=\mathfrak{q}$ be solvable with nilradical $\mathfrak{n}$ and let the subspace $\mathfrak{v} \subseteq \mathfrak{q}$ satisfy properties (I), (II) of Lemma 2.1. Define $\tau: \mathfrak{q} \rightarrow \mathcal{L}(\mathfrak{q})$ by $\tau(v+n) y=$ $-S(v) y$ for $v \in \mathfrak{v}, n \in \mathfrak{n}$, and $y \in \mathfrak{q}$. By Lemma 2.2, $\tau$ is a linear map and the $\tau(x), x \in \mathfrak{q}$, are mutually commuting derivations of $\mathfrak{q}$. Since $[\mathfrak{q}, \mathfrak{q}] \subseteq \mathfrak{n}$ and $\tau(n)=0$ for $n \in \mathfrak{n}$, it follows that $\tau$ is a representation. Moreover (10.4) holds because $S(v) y \in[\mathfrak{q}, \mathfrak{q}] \subseteq \mathfrak{n}$ and $\tau(n)=0$ for $n \in \mathfrak{n}$. The algebra $\mathfrak{a}^{\prime}=\left(\mathfrak{a},[\cdot, \cdot]^{\prime}\right)$ equals the nilshadow $\mathfrak{q}_{N}$ defined by relations $(2.3)$.

We study the Lie group analogue of Lemma 10.5. Let $A$ be a Lie group with product $*$, Lie algebra $\mathfrak{a}$ and exponential map exp, and suppose $T: A \rightarrow$ $\operatorname{Aut}(A)$ is a homomorphism into the group $\operatorname{Aut}(A)$ of smooth automorphisms of $A$. (We assume that $T$ is smooth in the sense that the map $(g, h) \mapsto T(g) h$ is smooth from $A \times A$ into $A$.) Thus $T(h) \in \operatorname{Aut}(A)$ for each $h \in A$, with $T(e)$ the identity automorphism, and $T\left(h_{1}\right) T\left(h_{2}\right)=T\left(h_{1} * h_{2}\right)$. If $\operatorname{Aut}(\mathfrak{a})$ is the group of automorphisms of $\mathfrak{a}$ then a homomorphism $\bar{T}: A \rightarrow \operatorname{Aut}(\mathfrak{a})$ is defined by

$$
\bar{T}(g) x=\left.\frac{d}{d t}\right|_{t=0} T(g)(\exp t x)
$$

for $g \in A, x \in \mathfrak{a}$. Then a Lie algebra representation $\tau: \mathfrak{a} \rightarrow \mathcal{L}(\mathfrak{a})$ of $\mathfrak{a}$ in itself by derivations is given by

$$
\tau(y) x=\left.\frac{d}{d t}\right|_{t=0} \bar{T}(\exp t y) x
$$

for $x, y \in \mathfrak{a}$. 
Let us make a note on identifications. The Lie algebra $\mathfrak{a}$ is the space of (real) left invariant vector fields on $A$, equipped with the commutator bracket; when convenient we identify $\mathfrak{a}=T_{e} A$, the tangent space of $A$ at the identity, by identifying $x \in T_{e} A$ with its unique extension to a left invariant vector field. With this convention, the differential operator $d L_{A}(x), x \in \mathfrak{a}$, is the right-invariant vector field whose value at $e$ is $-x$.

Lemma 10.6 let $T: A \rightarrow \operatorname{Aut}(A)$ be a smooth homomorphism and define $\bar{T}$ and $\tau$ as above. Suppose

$$
T(T(g) h)=T(h)
$$

for all $g, h \in A$. Then

(I) The operation $*^{\prime}$ defined by

$$
g *^{\prime} h=\left(T\left(h^{-1}\right) g\right) * h
$$

is a Lie group multiplication on the manifold $A$. For the Lie group $A^{\prime}=$ $\left(A, *^{\prime}\right)$ the identity element is $e^{\prime}=e$ and the inverse of $h$ is given by $h^{-1^{\prime}}=$ $T(h)\left(h^{-1}\right)$. Moreover $g *^{\prime} h^{-1^{\prime}}=T(h)\left(g * h^{-1}\right)$ for all $g, h \in A$.

(II) Let $\mathfrak{a}, \mathfrak{a}^{\prime}$ be the Lie algebras of $A$ and $A^{\prime}$ respectively. Identifying $\mathfrak{a}=T_{e} A=\mathfrak{a}^{\prime}$ as vector spaces, the Lie brackets $[\cdot, \cdot]$ of $\mathfrak{a}$ and $[\cdot, \cdot]^{\prime}$ of $\mathfrak{a}^{\prime}$ are related by

$$
[x, y]^{\prime}=[x, y]+\tau(x) y-\tau(y) x
$$

and $\tau(\tau(x) y)=0$ for all $x, y \in \mathfrak{a}$.

(III) The left regular representations of $A$ and $A^{\prime}$ are related by

$$
\left(\left(d L_{A^{\prime}}(x)\right) \varphi\right)(g)=\left(\left(d L_{A}\left(\bar{T}\left(g^{-1}\right) x\right)\right) \varphi\right)(g)
$$

for all $\varphi \in C_{c}^{\infty}(A), x \in \mathfrak{a}, g \in A$.

(IV) The maps $T, \bar{T}$ are homomorphisms of $A^{\prime}$, thus

$$
T\left(g *^{\prime} h\right)=T(g) T(h)=T(g * h) \quad, \quad \bar{T}\left(g *^{\prime} h\right)=\bar{T}(g) \bar{T}(h)=\bar{T}(g * h)
$$

for all $g, h \in A$.

(V) If $x \in \mathfrak{a}$ satisfies $\tau(x) x=0$ then $\exp (x)=\exp ^{\prime}(x)$, where exp, $\exp ^{\prime}$ are the exponential maps of $A, A^{\prime}$ respectively. 
Proof. The proof of (I) is by straightforward computations using (10.5). Here we just check the associative law: one has

$$
\begin{aligned}
g_{1} *^{\prime}\left(g_{2} *^{\prime} g_{3}\right) & =g_{1} *^{\prime}\left(T\left(g_{3}^{-1}\right) g_{2} * g_{3}\right) \\
& =T\left(g_{3}^{-1}\right) T\left(T\left(g_{3}^{-1}\right) g_{2}^{-1}\right) g_{1} *\left(T\left(g_{3}^{-1}\right) g_{2} * g_{3}\right) \\
& =T\left(g_{3}^{-1}\right) T\left(g_{2}^{-1}\right) g_{1} * T\left(g_{3}^{-1}\right) g_{2} * g_{3} \\
& =T\left(g_{3}^{-1}\right)\left(T\left(g_{2}^{-1}\right) g_{1} * g_{2}\right) * g_{3} \\
& =\left(g_{1} *^{\prime} g_{2}\right) *^{\prime} g_{3}
\end{aligned}
$$

for all $g_{1}, g_{2}, g_{3} \in A$.

For (III), if $g \in A$ let $R_{g}: A \rightarrow A$ be the right translation map $h \mapsto h * g$, and let $R_{g}^{\prime}$ be the right translation map $h \mapsto h *^{\prime} g$ relative to $A^{\prime}$. Let $d R_{g}, d R_{g}^{\prime}$ be the differentials of these maps. Regarding $d L_{A^{\prime}}(x)$ as a right-invariant vector field (see the remarks preceding the lemma), we have

$$
\left.d L_{A^{\prime}}(x)\right|_{g}=\left(d R_{g}^{\prime}\right)(-x)
$$

for all $g \in A, x \in \mathfrak{a}=T_{e} A$, and a similar result for $d L_{A}(x)$ with $R_{g}$ replacing $R_{g}^{\prime}$. But $R_{g}^{\prime}=R_{g} \circ\left(T\left(g^{-1}\right)\right)$, hence by differentiation

$$
d R_{g}^{\prime}=\left(d R_{g}\right) \circ\left(\bar{T}\left(g^{-1}\right)\right): T_{e} A \rightarrow T_{g} A,
$$

and we get

$$
\left.d L_{A^{\prime}}(x)\right|_{g}=\left(d R_{g}^{\prime}\right)(-x)=\left(d R_{g}\right)\left(-\bar{T}\left(g^{-1}\right) x\right)=\left.d L_{A}\left(\bar{T}\left(g^{-1}\right) x\right)\right|_{g},
$$

which is equivalent to (III).

For (II), let $x, y \in \mathfrak{a}=T_{e} A$, let $\varphi \in C_{c}^{\infty}(A)$, and set $X=d L_{A}(x)$, $X^{\prime}=d L_{A^{\prime}}(x), Y=d L_{A}(y), Y^{\prime}=d L_{A^{\prime}}(y)$. Then $\left.X^{\prime}\right|_{e}=\left.X\right|_{e}=-x$ so that

$$
\begin{aligned}
\left(X^{\prime} Y^{\prime} \varphi\right)(e) & =\left(X Y^{\prime} \varphi\right)(e) \\
& =\left.\frac{d}{d t}\right|_{t=0}\left(Y^{\prime} \varphi\right)(\exp (-t x)) \\
& =\left.\frac{d}{d t}\right|_{t=0}\left(d L_{A}(\bar{T}(\exp t x) y) \varphi\right)(\exp (-t x))
\end{aligned}
$$

by part (III), where exp is the exponential map of $A$. Setting

$$
F(s, t)=\left(d L_{A}(\bar{T}(\exp s x) y) \varphi\right)(\exp (-t x)),
$$

we have

$$
\begin{aligned}
\left(\partial_{s} F\right)(0,0) & =\left.\frac{d}{d s}\right|_{s=0}\left(d L_{A}(\bar{T}(\exp s x) y) \varphi\right)(e)=\left(d L_{A}(\tau(x) y) \varphi\right)(e), \\
\left(\partial_{t} F\right)(0,0) & =(X Y \varphi)(e) .
\end{aligned}
$$


It follows that

$$
\left(X^{\prime} Y^{\prime} \varphi\right)(e)=(X Y \varphi)(e)+\left(d L_{A}(\tau(x) y) \varphi\right)(e)
$$

Combining this with a similar expression for $\left(Y^{\prime} X^{\prime} \varphi\right)(e)$ we get

$$
\begin{aligned}
\left(d L_{A}([x, y]\right. & +\tau(x) y-\tau(y) x) \varphi)(e)= \\
& =((X Y-Y X) \varphi)(e)+\left(d L_{A}(\tau(x) y) \varphi\right)(e)-\left(d L_{A}(\tau(y) x) \varphi\right)(e) \\
& =\left(\left(X^{\prime} Y^{\prime}-Y^{\prime} X^{\prime}\right) \varphi\right)(e) \\
& =\left(d L_{A^{\prime}}\left([x, y]^{\prime}\right) \varphi\right)(e)=\left(d L_{A}\left([x, y]^{\prime}\right) \varphi\right)(e),
\end{aligned}
$$

and comparing the left and right sides of this equation yields (10.7). We leave the reader to verify that $\tau(\tau(x) y)=0$ for all $x$ and $y$, and then part (II) is proved.

For (IV) we note that

$$
T\left(g *^{\prime} h\right)=T\left(T\left(h^{-1}\right) g * h\right)=T\left(T\left(h^{-1}\right) g\right) T(h)=T(g) T(h),
$$

and the corresponding property for $\bar{T}$ follows by differentiation.

For $(\mathrm{V})$, suppose $\tau(x) x=0$. To prove that $\exp (t x)=\exp ^{\prime}(t x)$ for all $t \in \mathbb{R}$, it suffices to show that $t \mapsto \exp (t x)$ is a one-parameter subgroup of $A^{\prime}$. Noting for $s, t \in \mathbb{R}$ that

$$
T(\exp (-t x)) \exp s x=\exp (\bar{T}(\exp (-t x)) s x)=\exp \left(e^{-t \tau(x)} s x\right)=\exp s x \quad,
$$

it follows from the multiplication law (10.6) that

$$
\exp (s x) *^{\prime} \exp (t x)=\exp (s x) * \exp (t x)=\exp ((s+t) x) .
$$

This ends the proof of the lemma.

\subsection{Lie algebras with stratified nilshadow}

The following proposition shows that any (type $R$ ) Lie algebra is a quotient of a Lie algebra with stratified nilshadow (see Definition 2.6 for the definition of stratified nilshadow). This generalizes the well-known fact that any nilpotent algebra is a quotient of a stratified Lie algebra.

Proposition 10.7 Let $\mathfrak{g}$ be a Lie algebra of type $R$ with radical $\mathfrak{q}$ and nilradical $\mathfrak{n}$. There exists a Lie algebra $\tilde{\mathfrak{g}}$ of type $R$ which has stratified nilshadow $\tilde{\mathfrak{q}}_{N}$ with respect to a pair $(\tilde{\mathfrak{m}}, \tilde{\mathfrak{v}})$, and a surjective homomorphism $\pi: \tilde{\mathfrak{g}} \rightarrow \mathfrak{g}$. Moreover $\pi$ restricts to a homomorphism of $\tilde{\mathfrak{q}}_{N}$ onto the nilshadow $\mathfrak{q}_{N}$ of $\mathfrak{g}$, and $\pi\left(\tilde{\mathfrak{q}}_{N ; j}\right)=\mathfrak{q}_{N ; j}$ for all $j \in \mathbb{N}$. 
If $\tilde{\mathfrak{q}}, \tilde{\mathfrak{n}}$ denote respectively the radical and nilradical of $\tilde{\mathfrak{g}}$, then

$$
\pi^{-1}(\mathfrak{q})=\tilde{\mathfrak{q}} \quad, \quad \pi^{-1}(\mathfrak{n})=\tilde{\mathfrak{n}} .
$$

(Therefore if $\mathfrak{g}$ is respectively solvable or nilpotent, then $\tilde{\mathfrak{g}}$ possesses the same property.)

Proof. We divide the proof into three steps. In the first step we consider a stratified, "free nilpotent" Lie algebra $\mathfrak{r}$ such that $\mathfrak{q}_{N}$ is a quotient of $\mathfrak{r}$, and define certain semisimple derivations $R(v)$ of $\mathfrak{r}$. Second, from these derivations and Lemma 10.5 we construct a solvable Lie algebra $\tilde{\mathfrak{q}}$ such that $\tilde{\mathfrak{q}}=\mathfrak{r}$ as vector spaces, and show that $\mathfrak{r}$ is the nilshadow of $\tilde{\mathfrak{q}}$. Finally, we define $\tilde{\mathfrak{g}}$ as a semidirect product of $\tilde{\mathfrak{q}}$ with a Levi subalgebra of $\mathfrak{g}$.

Step 1. Fix a Levi subalgebra $\mathfrak{m}$ and a subspace $\mathfrak{v}$ of $\mathfrak{g}$ satisfying all properties of Lemma 2.1. Then the nilshadow $\mathfrak{q}_{N}$, the shadow $\mathfrak{g}_{N}$ and the representation $\tau: \mathfrak{g}_{N} \rightarrow \mathcal{L}\left(\mathfrak{g}_{N}\right)$ are defined as in Section 2. We choose $\tau$ invariant subspaces $\mathfrak{h}_{j}, j \in \mathbb{N}$, and $\mathfrak{k}_{1}=\mathfrak{h}_{1} \cap \mathfrak{n}$ as in Lemma 2.5. Fix a basis $b_{1}, \ldots, b_{d_{1}}$ of $\mathfrak{h}_{1}=\mathfrak{v} \oplus \mathfrak{k}_{1}$ such that $b_{1}, \ldots, b_{d_{0}}$ is a basis of $\mathfrak{v}$ and $b_{d_{0}+1}, \ldots, b_{d_{1}}$ is a basis of $\mathfrak{k}_{1}$. By Proposition $2.3, b_{1}, \ldots, b_{d_{1}}$ generate the Lie algebra $\mathfrak{q}_{N}$.

Suppose $\mathfrak{q}_{N}$ is step $r$ nilpotent. Let $\mathfrak{r}$ be the nilpotent Lie algebra with $d_{1}$ generators $\tilde{b}_{1}, \ldots, \tilde{b}_{d_{1}}$ which is free of step $r$, with Lie bracket $[\cdot, \cdot]_{\mathfrak{r}}$ (cf. [29], and Example 1.1.5 of [11]). More precisely, $\mathfrak{r}$ may be defined as the quotient $\mathcal{F} / \mathcal{I}$, where $\mathcal{F}$ is the free Lie algebra generated by $\tilde{b}_{1}, \ldots, \tilde{b}_{d_{1}}$ and $\mathcal{I}$ is the ideal of $\mathcal{F}$ spanned by all commutators $\left[\tilde{b}_{i_{1}},\left[\cdots,\left[\tilde{b}_{i_{s-1}}, \tilde{b}_{i_{s}}\right] \cdots\right]\right]$ with $s>r$. There is a surjective homomorphism $\pi: \mathfrak{r} \rightarrow \mathfrak{q}_{N}$ such that $\pi\left(\tilde{b}_{i}\right)=b_{i}$ for $i \in\left\{1, \ldots, d_{1}\right\}$.

The algebra $\mathfrak{r}$ decomposes as

$$
\mathfrak{r}=\bigoplus_{j=1}^{\infty} \tilde{\mathfrak{h}}_{j}=\bigoplus_{j=1}^{r} \tilde{\mathfrak{h}}_{j} \quad
$$

where $\tilde{\mathfrak{h}}_{j}$ is spanned by all commutators $\left[\tilde{b}_{i_{1}},\left[\cdots,\left[\tilde{b}_{i_{j-1}}, \tilde{b}_{i_{j}}\right]_{\mathfrak{r}} \cdots\right]_{\mathfrak{r}}\right]_{\mathfrak{r}}$ of length $j$. This decomposition is a stratification of $\mathfrak{r}$ in the sense of [25], that is, $\tilde{\mathfrak{h}}_{1}$ generates $\mathfrak{r}$ and $\left[\tilde{\mathfrak{h}}_{j}, \tilde{\mathfrak{h}}_{k}\right]_{\mathfrak{r}} \subseteq \tilde{\mathfrak{h}}_{j+k}$ for all $j, k \in \mathbb{N}$.

Let us set $\tilde{\mathfrak{v}}=\operatorname{span}\left\{\tilde{b}_{1}, \ldots, \tilde{b}_{d_{0}}\right\}, \tilde{\mathfrak{k}}_{1}=\operatorname{span}\left\{\tilde{b}_{d_{0}+1}, \ldots, \tilde{b}_{d_{1}}\right\}$, and $\tilde{\mathfrak{n}}=$ $\tilde{\mathfrak{k}_{1}} \oplus[\mathfrak{r}, \mathfrak{r}]_{\mathfrak{r}}$. Then $\tilde{\mathfrak{h}}_{1}=\tilde{\mathfrak{v}} \oplus \tilde{\mathfrak{k}}_{1}$ and

$$
\mathfrak{r}=\tilde{\mathfrak{h}}_{1} \oplus[\mathfrak{r}, \mathfrak{r}]_{\mathfrak{r}}=\tilde{\mathfrak{v}} \oplus \tilde{\mathfrak{k}}_{1} \oplus[\mathfrak{r}, \mathfrak{r}]_{\mathfrak{r}}=\tilde{\mathfrak{v}} \oplus \tilde{\mathfrak{n}}
$$

Observe that $\pi$ restricts to a linear bijection $\pi_{1}: \tilde{\mathfrak{h}}_{1} \rightarrow \mathfrak{h}_{1}$, and that $\pi^{-1}(\mathfrak{n})=\tilde{\mathfrak{n}}$. Define linear transformations $\widehat{R}(v) \in \mathcal{L}\left(\tilde{\mathfrak{h}}_{1}\right)$ by

$$
\widehat{R}(v)=\pi_{1}^{-1} \circ S\left(\pi_{1} v\right) \circ \pi_{1}
$$

for $v \in \tilde{\mathfrak{v}}$. 
It follows using Lemma 2.2 that the $\widehat{R}(v), v \in \tilde{\mathfrak{v}}$, are mutually commuting, semisimple transformations such that $\widehat{R}(v) w=0$ for all $v, w \in \tilde{\mathfrak{v}}$, and the mapping $v \mapsto \widehat{R}(v)$ is linear on $\tilde{\mathfrak{v}}$. Since $\mathfrak{g}$ is type $R$, the $S\left(\pi_{1} v\right)$ and hence $\widehat{R}(v)$ have only purely imaginary eigenvalues.

Consider a fixed $v \in \tilde{\mathfrak{v}}$. Because $\mathfrak{r}$ is free nilpotent on $\tilde{b}_{1}, \ldots, \tilde{b}_{d_{1}}$, the transformations $e^{t \widehat{R}(v)} \in \mathcal{L}\left(\tilde{\mathfrak{h}}_{1}\right), t \in \mathbb{R}$, extend uniquely to automorphisms $A(t)$ of $\mathfrak{r}$ such that $A(s) A(t)=A(s+t)$ for all $s, t \in \mathbb{R}$. Then

$$
R(v)=\lim _{t \rightarrow 0} t^{-1}(A(t)-I)
$$

defines a derivation $R(v)$ of $\mathfrak{r}$ whose restriction to $\tilde{\mathfrak{h}}_{1}$ equals $\widehat{R}(v)$. Combining the derivation property and the fact that $\tilde{b}_{1}, \ldots, \tilde{b}_{d_{1}}$ generate $\mathfrak{r}$, one verifies the following properties. The $R(v), v \in \tilde{\mathfrak{v}}$, are mutually commuting, semisimple derivations of $\mathfrak{r}$ with only purely imaginary eigenvalues, $R(v)(\tilde{\mathfrak{v}})=\{0\}, R(v)\left(\tilde{\mathfrak{k}}_{1}\right) \subseteq \tilde{\mathfrak{k}}_{1}, R(v)\left(\tilde{\mathfrak{h}}_{j}\right) \subseteq \tilde{\mathfrak{h}}_{j}$ for all $j \in \mathbb{N}$, and the mapping $v \mapsto R(v)$ is linear. (To verify the semisimplicity and the eigenvalue property, one may extend $R(v)$ to a derivation $R(v)^{\mathbb{C}}$ of the complexification $\mathfrak{r}^{\mathbb{C}}$ of $\mathfrak{r}$.) Moreover

$$
\pi \circ R(v)=S(\pi v) \circ \pi
$$

for all $v \in \tilde{\mathfrak{v}}$.

Step 2. Define $\tilde{\tau}: \mathfrak{r} \rightarrow \mathcal{L}(\mathfrak{r})$ by setting

$$
\tilde{\tau}(v+n)=R(v)
$$

for all $v \in \tilde{\mathfrak{v}}, n \in \tilde{\mathfrak{n}}$. It follows from the properties of $R(v)$ derived in Step 1 that $\tilde{\tau}$ is a representation of $\mathfrak{r}$ in itself by derivations. Since $\tilde{\tau}(x) y \in \tilde{\mathfrak{n}}$ for all $x, y \in \mathfrak{r}$, and $\tilde{\tau}(n)=0$ for all $n \in \tilde{\mathfrak{n}}$, we have $\tilde{\tau}(\tilde{\tau}(x) y)=0$. According to Lemma 10.5 , there is a Lie bracket $[\cdot, \cdot]$ on the vector space $\mathfrak{r}$ with

$$
[x, y]=[x, y]_{\mathfrak{r}}+\tilde{\tau}(x) y-\tilde{\tau}(y) x
$$

for $x, y \in \mathfrak{r}$; we denote by $\tilde{\mathfrak{q}}$ the Lie algebra $(\mathfrak{r},[\cdot, \cdot])$. Note that

$$
\left[v_{1}, v_{2}\right]=\left[v_{1}, v_{2}\right]_{\mathfrak{r}},\left[v_{1}, n_{1}\right]=\left[v_{1}, n_{1}\right]_{\mathfrak{r}}+R\left(v_{1}\right) n_{1},\left[n_{1}, n_{2}\right]=\left[n_{1}, n_{2}\right]_{\mathfrak{r}}
$$

for all $v_{1}, v_{2} \in \tilde{\mathfrak{v}}$ and $n_{1}, n_{2} \in \tilde{\mathfrak{n}}$. Recall that $\pi$ is a homomorphism of $\mathfrak{r}$ onto $\mathfrak{q}_{N}$. Then comparing (10.10) with (2.3), it follows from (10.9) and $\pi(\tilde{\mathfrak{v}})=\mathfrak{v}, \pi(\tilde{\mathfrak{n}})=\mathfrak{n}$, that $\pi$ is a homomorphism of $\tilde{\mathfrak{q}}$ onto $\mathfrak{q}$.

Note that $[x, y] \in \tilde{\mathfrak{n}}$ for all $x, y \in \tilde{\mathfrak{q}}$ and that the brackets $[\cdot, \cdot]$ and $[\cdot, \cdot]_{\mathfrak{r}}$ agree on $\tilde{\mathfrak{n}}$. Therefore $\tilde{\mathfrak{n}}$ contains $[\tilde{\mathfrak{q}}, \tilde{\mathfrak{q}}]$ and is a nilpotent ideal of $\tilde{\mathfrak{q}}$. 
In particular $[\tilde{\mathfrak{q}}, \tilde{\mathfrak{q}}]$ is nilpotent, so that $\tilde{\mathfrak{q}}$ is solvable. Let $\mathfrak{n}(\tilde{\mathfrak{q}})$ denote the nilradical of $\tilde{\mathfrak{q}}$. Since $\pi(\mathfrak{n}(\tilde{\mathfrak{q}}))$ is a nilpotent ideal of $\mathfrak{q}$ we have $\pi(\mathfrak{n}(\tilde{\mathfrak{q}})) \subseteq \mathfrak{n}$, that is, $\mathfrak{n}(\tilde{\mathfrak{q}}) \subseteq \pi^{-1}(\mathfrak{n})$. Because $\pi^{-1}(\mathfrak{n})=\tilde{\mathfrak{n}}$ and $\tilde{\mathfrak{n}}$ is a nilpotent ideal of $\tilde{\mathfrak{q}}$, we conclude that $\mathfrak{n}(\tilde{\mathfrak{q}})=\tilde{\mathfrak{n}}$.

Let $\operatorname{ad}_{\tilde{\mathfrak{q}}}$ and $\operatorname{ad}_{\mathfrak{r}}$ respectively denote the adjoint representations of $\tilde{\mathfrak{q}}$ and $\mathfrak{r}$, and let $\operatorname{ad}_{\tilde{\mathfrak{q}}} x=K(x)+S(x)$ denote the Jordan decomposition of $\operatorname{ad}_{\tilde{\mathfrak{q}}} x$ for $x \in \tilde{\mathfrak{q}}$.

Lemma 10.8 For $v \in \tilde{\mathfrak{v}}$ one has $K(v)=\operatorname{ad}_{\mathfrak{r}} v, S(v)=R(v)$.

Proof. It follows from (10.10) and the fact that $R\left(v_{1}\right) v_{2}=0$ for all $v_{1}, v_{2} \in \tilde{\mathfrak{v}}$ that $\operatorname{ad}_{\tilde{\mathfrak{q}}} v=\operatorname{ad}_{\mathfrak{r}} v+R(v)$ for $v \in \tilde{\mathfrak{v}}$. Moreover $\operatorname{ad}_{\mathfrak{r}} v, R(v)$ are respectively nilpotent and semisimple transformations. Since $R(v) v=0$ and $R(v)$ is a derivation of $\mathfrak{r}$ we have

$$
R(v)\left([v, x]_{\mathfrak{r}}\right)=[v, R(v) x]_{\mathfrak{r}}
$$

for all $x \in \mathfrak{r}$. Thus $R(v)$ commutes with $\operatorname{ad}_{\mathfrak{r}} v$, and the lemma follows from the uniqueness of the Jordan decomposition.

Let $\tilde{\mathfrak{w}}$ denote the subalgebra of $\tilde{\mathfrak{q}}$ generated by the subspace $\tilde{\mathfrak{v}}$; we claim that $\tilde{\mathfrak{w}}$ is nilpotent. Since $R(v)=S(v)$ is a derivation of $\tilde{\mathfrak{q}}$, and $R(v)(\tilde{\mathfrak{v}})=$ $\{0\}$, it follows that $R(v)(\tilde{\mathfrak{w}})=\{0\}$. Then it easily follows from (10.10) that $\left[w_{1}, w_{2}\right]=\left[w_{1}, w_{2}\right]_{\mathfrak{r}}$ for all $w_{1}, w_{2} \in \tilde{\mathfrak{w}}$. Thus $\tilde{\mathfrak{w}}$ is nilpotent.

Since $\tilde{\mathfrak{q}}=\tilde{\mathfrak{v}} \oplus \tilde{\mathfrak{n}}$ and $\tilde{\mathfrak{n}}$ is the nilradical of $\tilde{\mathfrak{q}}$, at this stage we have established that $\tilde{\mathfrak{v}}$ satisfies properties (I), (II) of Lemma 2.1 relative to $\tilde{\mathfrak{q}}$. Hence the nilshadow $\tilde{\mathfrak{q}}_{N}$ of $\tilde{\mathfrak{q}}$ is well-defined, with Lie bracket given by relations (2.3) relative to $\tilde{\mathfrak{v}}$ and $\tilde{\mathfrak{q}}$. From Lemma 10.8 and (10.10) we see that $\tilde{\mathfrak{q}}_{N}=\mathfrak{r}$ as Lie algebras. Note that $\tilde{\mathfrak{h}}_{1}=\tilde{\mathfrak{v}} \oplus\left(\tilde{\mathfrak{h}}_{1} \cap \tilde{\mathfrak{n}}\right)$, and the transformations $S(v)=R(v)=\tilde{\tau}(v+n)$ map $\tilde{\mathfrak{h}}_{j}$ into itself for all $j \in \mathbb{N}$ and $v \in \tilde{\mathfrak{v}}, n \in \tilde{\mathfrak{n}}$. Thus the conditions of Definition 2.6 are satisfied by $\tilde{\mathfrak{q}}$ and the subspaces $\tilde{\mathfrak{h}}_{j}$.

In summary, we have a solvable Lie algebra $\tilde{\mathfrak{q}}$, with stratified nilshadow $\tilde{\mathfrak{q}}_{N}$ with respect to $\tilde{\mathfrak{v}}$, and a surjective homomorphism $\pi: \tilde{\mathfrak{q}} \rightarrow \mathfrak{q}$. Moreover $\tilde{\mathfrak{q}}$ has nilradical $\tilde{\mathfrak{n}}=\pi^{-1}(\mathfrak{n})$. We leave the reader to verify that $\tilde{\mathfrak{q}}$ is of type $R$, using that $S(v)=R(v)$ has only purely imaginary eigenvalues for $v \in \tilde{\mathfrak{v}}$. Recalling that $\pi$ is a homomorphism of $\mathfrak{r}=\tilde{\mathfrak{q}}_{N}$ onto $\mathfrak{q}_{N}$, it follows that $\pi\left(\tilde{\mathfrak{q}}_{N ; j}\right)=\mathfrak{q}_{N ; j}$ for all $j \in \mathbb{N}$.

Step 3. The arguments of this step are similar to those of Steps 1 and 2, and we leave some verifications to the reader. Define a representation $\sigma$ of $\mathfrak{m}$ in $\tilde{\mathfrak{h}}_{1}$ by

$$
\sigma(x) y=\pi_{1}^{-1}\left(\left[x, \pi_{1} y\right]\right)
$$

for $x \in \mathfrak{m}, y \in \tilde{\mathfrak{h}}_{1}$. 
Each $\sigma(x)$ extends uniquely to a derivation of $\mathfrak{r}$ which we continue to denote $\sigma(x)$, and $\sigma(x)\left(\tilde{\mathfrak{h}}_{j}\right) \subseteq \tilde{\mathfrak{h}}_{j}$ for all $j \in \mathbb{N}$. Using $[\mathfrak{m}, \mathfrak{v}]=\{0\}$ one verifies that $\sigma(x) v=0$ and $[\sigma(x), S(v)]=0$ for all $v \in \tilde{\mathfrak{v}}$. Then it is easy to check that $\sigma(x)$ is a derivation of $\tilde{\mathfrak{q}}$. Also $\sigma: x \mapsto \sigma(x)$ is a representation of $\mathfrak{m}$ in $\tilde{\mathfrak{q}}$ by derivations, such that

$$
\pi(\sigma(x) y)=[x, \pi y]
$$

for all $x \in \mathfrak{m}, y \in \tilde{\mathfrak{q}}$.

Let $\tilde{\mathfrak{g}}$ be the semidirect product $\tilde{\mathfrak{g}}=\mathfrak{m} \times_{\sigma} \tilde{\mathfrak{q}}$, and identify $\tilde{\mathfrak{g}}=\mathfrak{m} \oplus \tilde{\mathfrak{q}}$ as vector spaces. Then the Lie bracket on $\tilde{\mathfrak{g}}$ is given by $\left[x_{1}+q_{1}, x_{2}+q_{2}\right]=$ $\left[x_{1}, x_{2}\right]+\left[q_{1}, q_{2}\right]+\sigma\left(x_{1}\right) q_{2}-\sigma\left(x_{2}\right) q_{1}$ for $x_{1}, x_{2} \in \mathfrak{m}, q_{1}, q_{2} \in \tilde{\mathfrak{q}}$. One checks that $\tilde{\mathfrak{g}}$ is type $R$. Extend $\pi: \tilde{\mathfrak{q}} \rightarrow \mathfrak{q}$ to a map $\pi: \tilde{\mathfrak{g}} \rightarrow \mathfrak{g}$ by setting $\pi(x+$ $q)=x+\pi(q)$ for $x \in \mathfrak{m}, q \in \tilde{\mathfrak{q}}$. Using (10.11) one obtains that $\pi$ is a homomorphism of $\tilde{\mathfrak{g}}$ onto $\mathfrak{g}$.

Note that $\tilde{\mathfrak{q}}$ and $\mathfrak{m}$ are respectively the radical and a Levi subalgebra of $\tilde{\mathfrak{g}}$, and that $[\mathfrak{m}, \tilde{\mathfrak{v}}]=\{0\}$. It is now easy to see that $\tilde{\mathfrak{g}}$ has stratified nilshadow $\tilde{\mathfrak{q}}_{N}$ with respect to $(\mathfrak{m}, \tilde{\mathfrak{v}})$ (with the stratification given by $\left\{\tilde{\mathfrak{h}}_{j}\right\}$ ).

The properties $\pi^{-1}(\mathfrak{q})=\tilde{\mathfrak{q}}, \pi^{-1}(\mathfrak{n})=\tilde{\mathfrak{n}}$ are easy consequences of the construction, and the proposition is proved.

\section{References}

[1] Alexopoulos, G.: An application of homogenization theory to harmonic analysis: Harnack inequalities and Riesz transforms on Lie groups of polynomial growth. Can. J. Math. 44 (1992), 691-727.

[2] Alexopoulos, G.: Sub-Laplacians with drift on Lie groups of polynomial volume growth. Mem. Amer. Math. Soc. 155 (2002), no. 739.

[3] Auscher, P., Elst, A.F. M. ter and Robinson, D. W.: On positive Rockland operators. Colloq. Math. 67 (1994), 197-216.

[4] Barnes, D. W.: On Cartan subalgebras of Lie algebras. Math. Z., 101: 350-355, 1967.

[5] Bourbaki, N.: Elements de mathematique: Groupes and algebres de Lie. Hermann, Paris, 1975.

[6] Brocker, T. And Dieck, T. tom: Representations of compact Lie groups. Graduate Texts in Mathematics 98. Springer-Verlag, New York, 1985.

[7] Butzer, P. L. And Berens, H.: Semi-groups of operators and approximation. Die Grundlehren der mathematischen Wissenschaften 145. SpringerVerlag, Berlin, 1967.

[8] Coifman, R. R. And Weiss, G.: Analyse harmonique non-commutative sur certains espaces homogénes. Lect. Notes in Math 242. Springer-Verlag, Berlin, 1971. 
[9] Coifman, R. R. And Weiss, G.: Transference methods in analysis. CBMS Regional Conference Series in Mathematics 31. Amer. Math. Soc., Providence, 1977.

[10] Corduneanu, C.: Almost-periodic functions. Interscience Publishers, New York, 1968.

[11] Corwin, L. and Greenleaf, F. P.: Representations of nilpotent Lie groups and their applications: Part I. Cambridge Studies in Advanced Mathematics 18. Cambridge University Press, 1990.

[12] David, G. And Journé, J. L.: A boundedness criterion for generalised Calderón-Zygmund operators. Ann. of Math. (2) 120 (1984), 371-397.

[13] Dungey, N.: Higher order operators and Gaussian bounds on Lie groups of polynomial growth. J. Operator Theory 46 (2001), 45-61.

[14] Dungey, N., Elst, A.F. M. ter and Robinson, D. W.: Asymptotics of sums of subcoercive operators. Colloq. Math. 82 (1999), 231-260.

[15] Dungey, N., Elst, A.F.M. ter and Robinson, D. W.: Analysis on Lie groups with polynomial growth. Birkhauser, Boston, 2003.

[16] Dziubanski, J., Hebisch, W. and Zienkiewicz, J.: Note on semigroups generated by positive Rockland operators on graded homogeneous groups. Studia Math. 110 (1994), 115-126.

[17] Elst, A. F. M. ter and Robinson, D. W.: Subelliptic operators on Lie groups: regularity. J. Austral. Math. Soc. Ser. A 57 (1994), 179-229.

[18] Elst, A. F. M. Ter and Robinson, D. W.: Subcoercivity and subelliptic operators on Lie groups I: Free nilpotent groups. Potential Anal. 3 (1994), 283-337.

[19] Elst, A. F. M. Ter And Robinson, D. W.: Local lower bounds on heat kernels. Positivity 2 (1998), 123-151.

[20] Elst, A. F. M. ter and Robinson, D. W.: Weighted subcoercive operators on Lie groups. J. Funct. Anal. 157 (1998), 88-163.

[21] Elst, A. F. M. Ter and Robinson, D. W.: Gaussian bounds for complex subelliptic operators on Lie groups of polynomial growth. Bull. Austral. Math. Soc. 67 (2003), 201-218.

[22] Elst, A. F. M. ter; Robinson, D. W. And Sikora, A.: Heat kernels and Riesz transforms on nilpotent Lie groups. Colloq. Math. 74 (1997), $191-218$.

[23] Elst, A. F. M. ter; Robinson, D. W. and Sikora, A.: Riesz transforms and Lie groups of polynomial growth. J. Funct. Anal. 162 (1999), $14-51$.

[24] Folland, G. B.: A course in abstract harmonic analysis. CRC Press, Florida, 1995.

[25] Folland, G. B. and Stein, E. S.: Hardy spaces on homogeneous groups. Mathematical Notes 28. Princeton University Press, Princeton, 1982. 
[26] Guivarc'H, Y.: Croissance polynomiale et périodes des fonctions harmoniques. Bull. Soc. Math. France 101 (1973), 333-379.

[27] Helffer, B. And Nourrigat, J.: Caractérisation des opérateurs hypoelliptiques homogènes invariants à gauche sur un groupe de Lie nilpotent gradué. Comm. Partial Differential Equations 4 (1979), 899-958.

[28] Kato T.: Perturbation theory for linear operators, second edition. Die Grundlehren der mathematischen Wissenschaften 132. Springer-Verlag, Berlin, 1984.

[29] Nagel, A., Ricci, F. And Stein, E. M.: Harmonic analysis and fundamental solutions on nilpotent Lie groups. In Analysis and partial differential equations, 249-275. Lecture Notes in Pure and Appl. Math. 122. Marcel Dekker, New York, 1990.

[30] Robinson, D. W.: Elliptic operators and Lie groups. Oxford University Press, Oxford, 1991.

[31] SAloff-Coste, L.: Analyse sur les groupes de Lie à croissance polynômiale. Ark. Mat. 28 (1990), 315-331.

[32] Varadarajan, V.S.: Lie Groups, Lie Algebras, and their Representations. Graduate Texts in Mathematics 102. Springer-Verlag, New York, 1984.

[33] Varopoulos, N. T.: Analysis on nilpotent groups. J. Funct. Anal. 66 (1986), 406-431.

[34] Varopoulos, N. T.: Analysis on Lie groups. J. Funct. Anal. 76 (1988), 346-410.

[35] Varopoulos, N. T., Saloff-Coste, L. and Coulhon, T.: Analysis and geometry on groups. Cambridge Tracts in Mathematics 100. Cambridge University Press, Cambridge, 1992.

[36] Winter, D. J.: Abstract Lie algebras. The M.I.T. Press, Cambridge, Mass.-London, 1972.

Recibido: 28 de julio de 2003

Nick Dungey

School of Mathematics University of New South Wales

Sydney 2052 Australia dungey@maths. unsw . edu . au

During this work the author was located in the Mathematical Sciences Institute at the Australian National University, and was supported by an Australian Research Council (ARC) Postdoctoral Fellowship. I thank Tom ter Elst and Derek Robinson for many valuable discussions and for suggesting a number of improvements. 